\title{
A port-reduced static condensation reduced basis element method for large component- synthesized structures: approximation and $A$ Posteriori error estimation
}

\author{
Jens L Eftang ${ }^{1,2^{*}}$ and Anthony T Patera ${ }^{1}$
}

\author{
${ }^{*}$ Correspondence: \\ jleftang@gmail.com \\ ${ }^{1}$ Department of Mechanical \\ Engineering, Massachusetts \\ Institute of Technology, 77 \\ Massachusetts Avenue, Cambridge, \\ MA-02139, USA \\ ${ }^{2}$ Current address: DNV \\ GL - Software, P.O.Box 300, \\ NO-1322, Høvik, Norway
}

\begin{abstract}
Background: We consider a static condensation reduced basis element framework for efficient approximation of parameter-dependent linear elliptic partial differential equations in large three-dimensional component-based domains. The approach features an offline computational stage in which a library of interoperable parametrized components is prepared; and an online computational stage in which these component archetypes may be instantiated and connected through predefined ports to form a global synthesized system. Thanks to the component-interior reduced basis approximations, the online computation time is often relatively small compared to a classical finite element calculation.
\end{abstract}

Methods: In addition to reduced basis approximation in the component interiors, we employ in this paper port reduction with empirical port modes to reduce the number of degrees of freedom on the ports and thus the size of the Schur complement system. The framework is equipped with efficiently computable a posteriori error estimators that provide asymptotically rigorous bounds on the error in the approximation with respect to the underlying finite element discretization. We extend our earlier approach for two-dimensional scalar problems to the more demanding three-dimensional vector-field case.

Results and Conclusions: This paper focuses on linear elasticity analysis for large structures with tens of millions of finite element degrees of freedom. Through our procedure we effectively reduce the number of degrees of freedom to a few thousand, and we demonstrate through extensive numerical results for a microtruss structure that our approach provides an accurate, rapid, and a posteriori verifiable approximation for relevant large-scale engineering problems.

Keywords: Static condensation; Reduced basis element method; Component synthesis; Domain decomposition; Port reduction; Interface reduction; A posteriori error estimation; Non-conforming methods; Structural analysis; Large-scale simulation 


\section{Background}

For several decades the finite element (FE) method has been a popular and important tool in engineering design and analysis of systems modelled by partial differential equations (PDEs). In particular, in fields such as structural analysis and strength assessment, the FE method is in widespread use in industry through a variety of commercial software packages. Many of the structures that are subject to industrial FE analysis are composed of a large number of components - consider for example a truss bridge, a space satellite [1], or a building or vehicle frame. Such large and at first sight complicated structures pose challenges both in terms of initial manual labor related to domain modelling and meshing, and in terms of subsequent computational cost.

Component-based structures which contain many identical or similar components are often analyzed through substructuring or superelement techniques [2], which mitigate some of these issues. Mathematically, superelement techniques are based on static condensation of all FE degrees of freedom that are interior to components, and hence the size of the global but condensed linear-algebraic (Schur complement) system is equal to the number of degrees of freedom associated with component interfaces, henceforth in this paper referred to as ports. The static condensation step necessitates a large number of component-interior FE "bubble" solves - one FE solve for each degree of freedom on each port of each component - and is for this reason rather expensive; however this step is embarrassingly parallel, and is furthermore required only once for each unique component instantiation.

Model order reduction techniques can be applied to substructuring or superelement procedures in order to further reduce the computational cost. A well-known approach is the classical component mode synthesis (CMS) [3,4], which replaces the original FE spaces for the component-interior bubble solves with spaces spanned by a few component-interior eigenmodes. As a result, the cost associated with each bubble calculation is reduced, and the formation of the global Schur complement system is consequently much less expensive.

A more recent approach, which is relevant in the context of parameter-dependent PDEs and which we for this reason consider here in this paper, is the static condensation reduced basis element method (SCRBE) introduced in [5]. Rather than the eigenmodal expansion typically used in the CMS, the SCRBE employs the reduced basis method (RB) [6] for the bubble function approximations. Each RB approximation space is specifically tailored to a particular bubble and the associated parameter dependence defined by the PDE within each component; the SCRBE thus accommodates parametric variations for example related to component geometry, loads, material properties, or boundary conditions. Furthermore, thanks to the typically very rapid (often exponential) convergence of the RB approximation $[7,8]$, these RB spaces are low-dimensional and thus bubble function approximation is computationally inexpensive.

In addition to enabling parametric variations, the SCRBE features a strict offline-online computational decoupling. In the offline stage, the RB spaces and associated datasets for each component archetype in a component library is computed and stored. This stage requires FE solves and may thus be relatively expensive, but is carried out only once as a library preprocessing step. In the subsequent online stage, the user may instantiate any of the interoperable library archetypes, and assign to each component instantiation the desired parameter values; the RB bubble function approximations are then computed, 
and the Schur complement system is assembled and solved. This online step is much less expensive and in particular does never invoke the underlying FE discretization.

However, common to all these static-condensation-based approaches - including the SCRBE - is a global Schur complement linear-algebraic system of size equal to the total number of degrees of freedom associated with ports. For large systems with many components and ports, and in particular for problems with three-dimensional vector-valued field variables - such as in linear elasticity - the size of this system is considerable and thus clearly prohibits the fast response required in, say, an interactive design or optimization context. To overcome this limitation various port reduction techniques may be used. For example, for the CMS approaches an eigenmode expansion (with subsequent truncation) for the port degrees of freedom is considered in $[9,10]$, and an adaptive procedure based on a posteriori error estimators for the port reduction is considered in [11]. For the SCRBE, we introduce in [12] port reduction with empirical modes; in this case the port approximation spaces are informed by snapshots of relevant port-restricted solutions which are obtained through an offline pairwise empirical training algorithm.

Unique to the SCRBE is a certification framework that allows efficient computation of $a$ posteriori bounds or estimators for the error in the SCRBE approximation with respect to the underlying FE "truth" discretization. This framework invokes classical residual arguments on the (RB) bubble level [6], a non-conforming approximation to the error-residual equation at the port level, and finally matrix perturbation at the system level in order to bound (under an eigenvalue proximity assumption) the error contributions from both RB approximation [5] and port reduction [12]. In actual practice, we may reduce online computational cost by consideration of a plausible and asymptotically rigorous error estimator rather than a rigorous error bound.

In this paper, we extend our earlier work for two-dimensional scalar problems in [12] to the more demanding three-dimensional vector-field case. We focus here on applications in linear elasticity, but we note that the component synthesis and indeed RB and port approximations can be readily extended to problems in heat transfer or (frequency domain) acoustics, or any phenomenon described by a linear elliptic or parabolic [13] $\mathrm{PDE}^{\mathrm{a}}{ }^{\mathrm{a}}$ Through our procedure we effectively reduce the number of degrees of freedom from tens of millions (in the underlying FE discretization) to only a few thousand (in the port-reduced SCRBE approximation); the associated computation time is thus reduced from minutes or hours to only a few seconds.

Our approach here features several important innovations. First, as we consider here larger global systems with a much larger number of instantiated components we introduce a new non-symmetric SCRBE approximation, which reduces both offline and online cost and memory footprint; the corresponding linear-algebraic system is subsequently symmetrized in order to (say) accommodate efficient linear solvers. We also demonstrate that our central theoretical results in particular related to a posteriori error estimation survive intact for this more efficient revision of our earlier formulations in [12]. Second, we provide a precise formulation for general geometric mappings and port space compatibility, and we demonstrate that (in the isotropic linear-elastic case) rigid-body parameters related to "docking" of component instantiations in a system do not affect the associated bilinear forms and thus do not impact offline - thanks to smaller RB space dimensions - or online - thanks to treatment of differently oriented component instantiations as effectively identical - computational cost. Third, we introduce a 
new functional interpretation of our algebraic a posteriori error estimation framework in [12], which may serve to extend our approach here to larger classes of problems. And finally, we consider multi-reference parameter bound conditioners [14] for sharper error estimation.

The remainder of the paper is organized as follows. We start with a brief presentation of a general parametrized component static condensation framework for $d$-dimensional vector-valued linear elliptic partial differential equations; we focus on the concepts relevant in the SCRBE framework and we formulate the port compatibility requirements. Next, we discuss the RB and port reduction strategies for the computational cost reduction associated with component interiors and component interfaces, respectively. Then, we introduce our a posteriori error estimation framework. Finally, we present extensive results for a three-dimensional microtruss application, and provide some conclusive remarks.

We include with this manuscript [Additional file 1]. This short movie presents the main ingredients of the port-reduced SCRBE method, and sums up the key numerical results reported in this paper.

\section{Component-based static condensation}

\section{Concepts: library components and system}

We now introduce the key concepts for our SCRBE approximation: a library of parametrized and interoperable archetype components, which is prepared in the offline stage; and a system of component instantiations connected at ports, which is assembled and solved (and, if desired, visualized) in the online stage.

In the context of structural analysis, an archetype component typically (but not necessarily) corresponds to a physical construction unit, such as a beam, a plate, or a connector; in physical $d$-dimensional space $(d=1,2,3)$ we denote by $\hat{\Omega}_{m} \subset \mathbb{R}^{d}$ the reference domain associated with archetype component $m, 1 \leq m \leq M$, where $M$ is the number of archetypes in the library. The boundary of this domain, $\partial \hat{\Omega}_{m}$, has a set of $n_{m}^{\gamma}$ disjoint local ports, denoted as $\hat{\gamma}_{m, j} \subseteq \partial \hat{\Omega}_{m}, 1 \leq j \leq n_{m}^{\gamma}$; these ports enable the components to connect to other components. Note we shall assume that all ports on an archetype component are mutually separated by (at least) a non-port, non-Dirichlet boundary segment. If this is not the case, modifications to our procedures below must be considered [10].

The physical behavior of each archetype component is governed by a vector-valued (we consider $d$ field components) parametrized linear elliptic partial differential equation. We thus introduce for $1 \leq m \leq M$ the continuous (and here, in this paper, symmetric) archetype bilinear form $\hat{a}_{m}\left(\cdot, \cdot ; \hat{\mu}_{m}\right):\left(H^{1}\left(\hat{\Omega}_{m}\right)\right)^{d} \times\left(H^{1}\left(\hat{\Omega}_{m}\right)\right)^{d} \rightarrow \mathbb{R}$, and the bounded archetype linear functional $\hat{f}_{m}\left(\cdot, \cdot ; \hat{\mu}_{m}\right):\left(H^{1}\left(\hat{\Omega}_{m}\right)\right)^{d} \rightarrow \mathbb{R}$. Here, $\hat{\mu}_{m} \in \hat{\mathcal{D}}^{m} \subset \mathbb{R}^{\hat{P}_{m}}$ is a vector of $\hat{P}_{m}$ scalar parameters that describe (say) the component geometry, boundary conditions, loads, or material properies, and $\left(H^{1}\left(\hat{\Omega}_{m}\right)\right)^{d}$ is the usual $(d$-tensorized) firstorder Sobolev space over $\hat{\Omega}_{m}$. We shall assume that $\hat{a}_{m}$ and $\hat{f}_{m}$ admit affine expansions as

$$
\hat{a}_{m}\left(\cdot, \cdot ; \hat{\mu}_{m}\right)=\sum_{q=1}^{\hat{Q}_{m}^{a}} \hat{a}^{q}(\cdot, \cdot) \Theta_{a}^{q}\left(\hat{\mu}_{m}\right), \quad \hat{f}_{m}\left(\cdot ; \hat{\mu}_{m}\right)=\sum_{q=1}^{\hat{Q}_{m}^{f}} \hat{f}^{q}(\cdot) \Theta_{f}^{q}\left(\hat{\mu}_{m}\right)
$$


where the $\hat{a}^{q}$ and $\hat{f}^{q}$ are parameter-independent forms and the $\Theta_{a}^{q}$ and $\Theta_{f}^{q}$ are parameterdependent functions; for computational efficiency of the SCRBE evaluation stage it is critical that $\hat{Q}_{m}^{a}$ and $\hat{Q}_{m}^{f}$ are relatively small.

We next introduce the discrete archetype component spaces $\hat{X}_{m}^{h} \subset\left(H^{1}\left(\hat{\Omega}_{m}\right)\right)^{d}, 1 \leq$ $m \leq M$, which correspond to standard FE discretizations [15] of $\left(H^{1}\left(\hat{\Omega}_{m}\right)\right)^{d}$; and we introduce the discrete port spaces, the restrictions

$$
\left.\hat{P}_{m, j} \equiv \hat{X}_{m}^{h}\right|_{\hat{\gamma}_{m, j}}
$$

of dimension $\mathcal{N}_{m}^{\gamma} \equiv \operatorname{dim}\left(\hat{P}_{m, j}\right)$. We denote the bases for these port spaces by $\left\{\hat{\chi}_{m, j, k}\right\}_{k=1}^{\mathcal{N}_{m, j}^{\gamma}}$ such that

$$
\hat{P}_{m, j}=\operatorname{span}\left\{\hat{\chi}_{m, j, 1}, \ldots, \hat{\chi}_{m, j, \mathcal{N}_{m, j}^{\gamma}}\right\} .
$$

For simplicity of presentation here we shall assume that Dirichlet conditions are enforced only on ports and thus not through the archetype component discrete spaces $\hat{X}_{m}^{h}$ (this is the case for our numerical results later).

The library component archetypes may be instantiated and connected at ports to form a global system. To this end we introduce a mapping $\mathcal{M}:\{1, \ldots, I\} \rightarrow\{1, \ldots, M\}$ from any of the $I$ instantiations in the system to exactly one of the $M$ archetypes in the library. For instantiated component $i$, we introduce the parameter vector $\mu_{i} \in \mathcal{D}_{i}$, where $\mathcal{D}_{i} \subseteq$ $\hat{\mathcal{D}}_{\mathcal{M}(i)}$. We then introduce a (parameter-dependent) geometric mapping $\mathcal{T}_{i}: \hat{\Omega}_{i} \rightarrow \Omega_{i}$ from archetype (reference) to system (physical) coordinates; thus $\Omega_{i}=\mathcal{T}_{i}\left(\hat{\Omega}_{\mathcal{M}(i)}\right)$ is the instantiated component domain and $\gamma_{i, j}=\mathcal{T}_{i}\left(\hat{\gamma}_{\mathcal{M}(i), j}\right), 1 \leq j \leq n_{\mathcal{M}(i)}^{\gamma}$, are the instantiated ports. We consider for each of our mappings $\mathcal{T}_{i}$ application of a deformation $\mathcal{T}_{i}^{\text {def }}$ and then a rotation $\mathcal{T}_{i}^{\text {rot }}$ such that $\mathcal{T}_{i} \equiv \mathcal{T}_{i}^{\text {rot }} \mathcal{T}_{i}^{\text {def }}$. In this paper, we consider for $\mathcal{T}_{i}^{\text {def }}$ only dilation and translation, and we further assume that $\mathcal{T}_{i}^{\text {def }}$, when applied to a port, is pure translation (such that $\gamma_{i, j}=\mathcal{T}_{i}\left(\hat{\gamma}_{\mathcal{M}(i), j}\right)$ corresponds to a rigid-body transformation). We illustrate the situation (for $d=2$ ) in Figure 1 and Figure 2: in Figure 1 we show a single

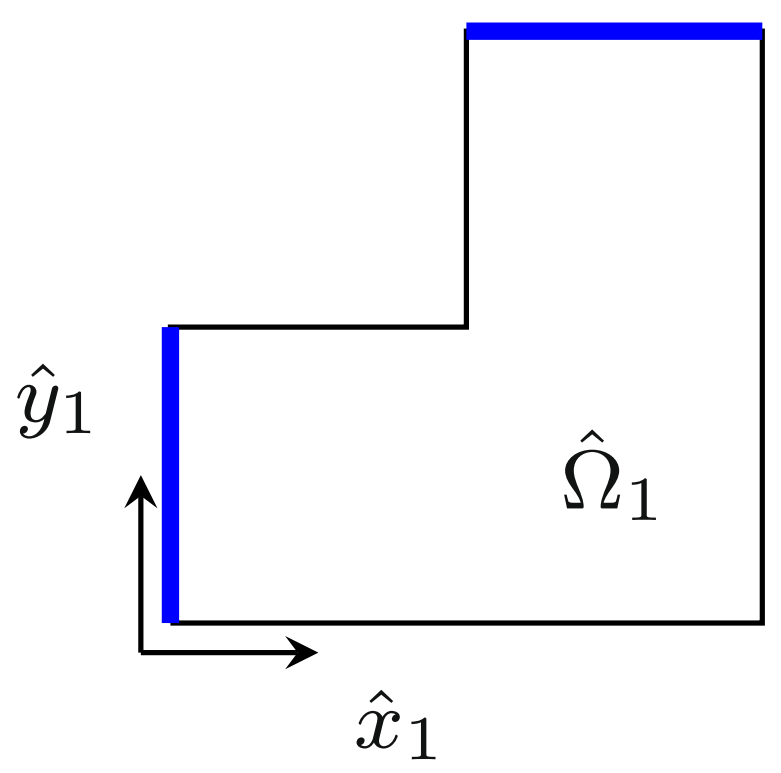

Figure 1 An archetype component in coordinates $\left(\hat{x}_{m}, \hat{y}_{m}\right)$. 


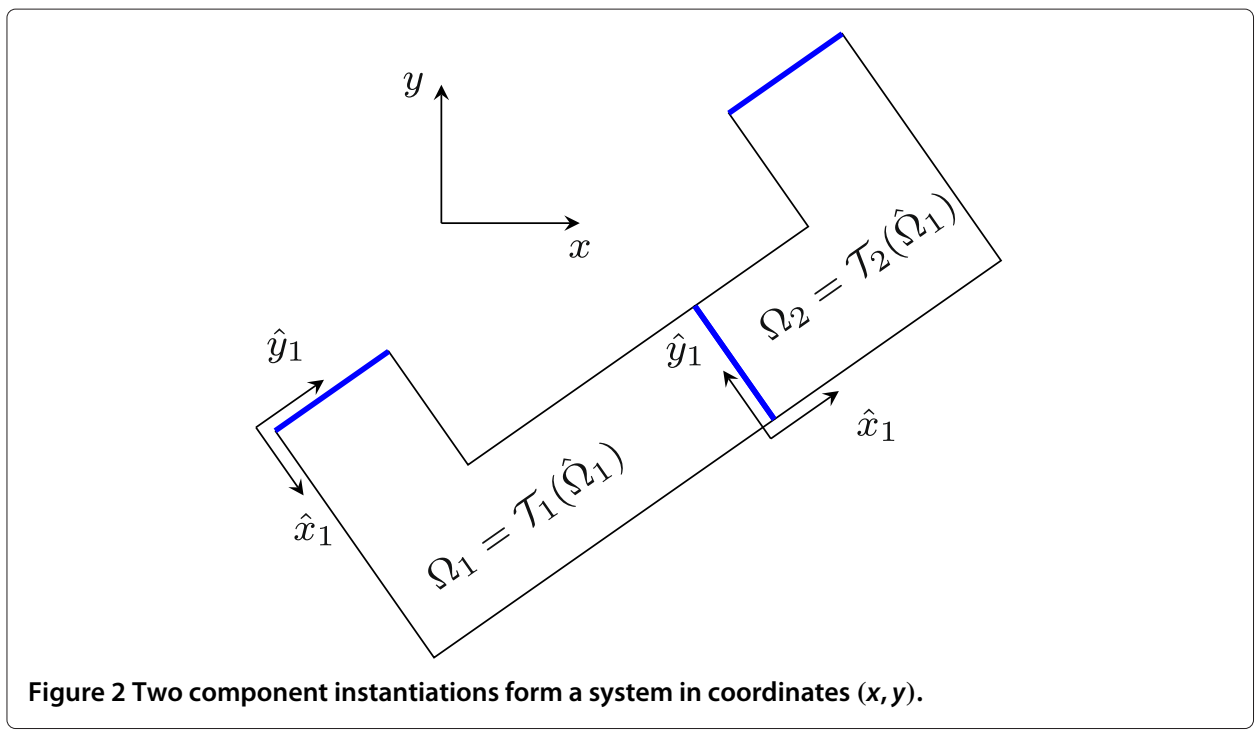

archetype library component; in Figure 2 we instantiate two components of the same archetype subject to different mappings $\mathcal{T}_{1}$ and $\mathcal{T}_{2}$, the first of which has a non-trivial (piecewise dilation) $\mathcal{T}_{1}^{\text {def }}$.

We also introduce a mapped discrete component-local space

$$
X_{i}^{h}=\operatorname{span}\left\{\mathcal{T}_{i}^{\mathrm{rot}}\left(v \circ \mathcal{T}_{i}^{-1}\right), \quad v \in \hat{X}_{\mathcal{M}(i)}^{h}\right\}
$$

and further, with

$$
\chi_{i, j, k} \equiv \mathcal{T}_{i}^{\text {rot }}\left(\hat{\chi}_{\mathcal{M}(i), j, k} \circ \mathcal{T}_{i}^{-1}\right),
$$

we introduce, for $1 \leq j \leq n_{\mathcal{M}(i)}^{\gamma}, 1 \leq i \leq I$, the mapped discrete port spaces

$$
P_{i, j}^{h}=\operatorname{span}\left\{\chi_{i, j, k}, \quad 1 \leq k \leq \mathcal{N}_{\mathcal{M}(i), j}^{\gamma}\right\} .
$$

Note that here and in the following the notation $[\cdot] \circ \mathcal{T}_{i}{ }^{-1}$ denotes the usual composition, ${ }^{b}$ and the notation $\mathcal{T}_{i}{ }^{\text {rot }}(\cdot)$ denotes pointwise application of $\mathcal{T}_{i}{ }^{\text {rot }}$ to the (vector-valued) argument; ${ }^{\mathrm{c}}$ we apply $\mathcal{T}_{i}^{\text {rot }}$ to the dependent variables to eliminate parameters related to spatial orientation of components from the bilinear forms, and to accommodate compatibility of basis functions on instantiated ports.

We may now introduce the synthesized system domain $\Omega$ as $\bar{\Omega}=\cup_{i=1}^{I} \bar{\Omega}_{i}$, the system parameter domain $\mathcal{D}=\oplus_{i=1}^{I} \mathcal{D}_{i}$, and the system parameter vector $\mu=\left(\mu_{1}, \ldots, \mu_{I}\right)$; we denote the total number of system parameters by $P$.

When an instantiated component becomes part of a system, its local ports are associated to global ports. Each global port $\Gamma_{p}, 1 \leq p \leq n_{0}^{\Gamma}$, in the system is either a coincidence of two local ports and hence in the interior of $\Omega$, or a single local port on the boundary $\partial \Omega$. We define the connectivity of the system through global-to-local index sets $\pi_{p}$, $1 \leq p \leq n_{0}^{\Gamma}$ : an interior global port is associated to two local ports $\gamma_{i, j}$ and $\gamma_{i^{\prime}, j^{\prime}}$, and we thus set $\pi_{p}=\left\{(i, j),\left(i^{\prime}, j^{\prime}\right)\right\}$; a boundary global port is associated to a single local port $\gamma_{i, j}$, and we thus set $\pi_{p}=\{(i, j)\}$. We also introduce for instantiated component $i, 1 \leq i \leq I$, a local-to-global map $\mathcal{G}_{i}$ such that for local port $j, 1 \leq j \leq n_{\mathcal{M}(i)}^{\gamma}$, we have $\mathcal{G}_{i}(j)=p$ if $(i, j) \in \pi_{p}$. Note that on any global port $\Gamma_{p}, 1 \leq p \leq n_{0}^{\Gamma}$, we may elect to impose Dirichlet 
boundary conditions; we denote by $n^{\Gamma} \leq n_{0}^{\Gamma}$ the number of global ports on which we do not impose Dirichlet boundary conditions.

To ensure global continuity of the solution we must require conforming port spaces and bases in the sense that for any shared (that is, interior) global port $\pi_{p}=\left\{(i, j),\left(i^{\prime}, j^{\prime}\right)\right\}$ we must have

$$
\chi_{i, j, k}=\chi_{i^{\prime}, j^{\prime}, k}
$$

we discuss this port compatibility requirement further in the "Port compatibility" subsection below.

We may now introduce for any $w, v \in\left(H^{1}(\Omega)\right)^{d}$ and any $\mu \in \mathcal{D}$ the system-level symmetric, continuous bilinear form as

$$
a(w, v ; \mu)=\sum_{i=1}^{I} \hat{a}_{\mathcal{M}(i)}\left(\left(\mathcal{T}_{i}^{\text {rot }}\right)^{-1}\left(\left.w\right|_{\Omega_{i}} \circ \mathcal{T}_{i}\right),\left(\mathcal{T}_{i}^{\text {rot }}\right)^{-1}\left(\left.v\right|_{\Omega_{i}} \circ \mathcal{T}_{i}\right) ; \mu_{i}\right),
$$

and the system-level bounded linear functional

$$
f(v ; \mu)=\sum_{i=1}^{I} \hat{f}_{\mathcal{M}(i)}\left(\left(\mathcal{T}_{i}^{\mathrm{rot}}\right)^{-1}\left(\left.v\right|_{\Omega_{i}} \circ \mathcal{T}_{i}\right) ; \mu_{i}\right) ;
$$

note that the effect of the mapping $\mathcal{T}_{i}$ to each archetype bilinear and linear form (defined over the archetype reference domain) is reflected through the parameter $\mu_{i}$.

In the case that $\mathcal{T}_{i}$ is a pure rigid-body transformation (that is, $\mathcal{T}_{i}$ is a rotation and a translation) and the material properties of the component do not depend on spatial orientation - such as in isotropic linear elasticity - the application of $\mathcal{T}^{\text {rot }}$ to the dependent variables results in cancellation of the mapping Jacobians, and thus the archetype bilinear form does not reflect the associated mapping parameters. Similarly, when $\mathcal{T}_{i}$ is a combination of a rigid-body map and (say) dilation, only the latter must be parametrized through the archetype bilinear form. We explicitly demonstrate this cancellation for the case of isotropic linear elasticity in the "Microtruss beam application" section, and we comment on the computational implications in the "Model reduction" section.

We now introduce a global space $X(\Omega) \subset\left(H^{1}(\Omega)\right)^{d}$ such that $X(\Omega)$ is equal to $\left(H^{1}(\Omega)\right)^{d}$ except for restrictions to enforce port (and in general also non-port) Dirichlet boundary conditions; we assume that sufficient boundary conditions are enforced such that $a(\cdot, \cdot ; \mu)$ is coercive over $X(\Omega)$. The well-posed system-level variational problem then reads as follows. For any $\mu \in \mathcal{D}$, find $u(\mu) \in X(\Omega)$ such that

$$
a(u(\mu), v ; \mu)=f(v ; \mu), \quad \forall v \in X(\Omega) ;
$$

we also introduce a compliance output as $s(\mu)=f(u(\mu) ; \mu)$. (Note that, as discussed in [5], restrictions apply to the geometric maps $\mathcal{T}_{i}$ to maintain well-posedness of (10).)

Similarly, we introduce a global FE discretization $X^{h}(\Omega) \subset X(\Omega)$ as $X^{h}(\Omega)=$ $\oplus_{i=1}^{I} X_{i}^{h}(\Omega) \cap X(\Omega)$; hence $X^{h}(\Omega)$ inherits the boundary conditions as well as the global continuity enforced by $X(\Omega)$. The FE discretization of (10) now reads as follows. For any $\mu \in \mathcal{D}$, find $u^{h}(\mu) \in X^{h}(\Omega)$ such that

$$
a\left(u^{h}(\mu), v ; \mu\right)=f(v ; \mu), \quad \forall v \in X^{h}(\Omega) ;
$$

we also introduce the FE compliance output $s^{h}(\mu)=f\left(u^{h}(\mu) ; \mu\right)$. 


\section{Mathematical formulation: static condensation}

To formulate the static condensation procedure we decompose our discrete global space $X^{h}(\Omega)$ into bubble spaces associated with component-interior degrees of freedom and a skeleton space associated with port degrees of freedom.

To this end we introduce on archetype component $m, 1 \leq m \leq M$, the bubble space

$$
\hat{B}_{m ; 0}^{h}=\left\{v \in \hat{X}_{m}^{h}:\left.v\right|_{\hat{\gamma}_{m, j}}=0, \quad 1 \leq j \leq n_{m}^{\gamma}\right\}
$$

note that members of $\hat{B}_{m ; 0}^{h}$ vanish on local ports. We next introduce the coupling modes $\hat{\psi}_{m, j, k} \in \hat{X}_{m}^{h}$ such that

$$
\begin{aligned}
\int_{\hat{\Omega}_{m}} \nabla \hat{\psi}_{m, j, k} \cdot \nabla v & =0, \quad \forall v \in \hat{B}_{m ; 0}^{h}, \\
\hat{\psi}_{m, j, k} & = \begin{cases}\hat{\chi}_{m, j, k}, & \text { on } \hat{\gamma}_{m, j}, \\
0, & \text { on } \hat{\gamma}_{m, j^{\prime}} \text { for } j^{\prime} \neq j,\end{cases}
\end{aligned}
$$

for $1 \leq k \leq \mathcal{N}_{m, j}^{\gamma}, 1 \leq j \leq n_{m}^{\gamma}$; we define, on instantiated component $i, 1 \leq i \leq I$, $\psi_{i, j, k} \equiv \mathcal{T}_{i}^{\operatorname{rot}}\left(\hat{\psi}_{\mathcal{M}(i), j, k} \circ \mathcal{T}_{i}^{-1}\right)$, and we introduce the global functions $\Psi_{p, k} \in X^{h}(\Omega)$ such that, for $\pi_{p}=\left\{(i, j),\left(i^{\prime}, j^{\prime}\right)\right\}$,

$$
\Psi_{p, k} \equiv \begin{cases}\psi_{i^{\prime}, j^{\prime}, k}, & \text { in } \Omega_{i^{\prime}}, \\ \psi_{i, j, k}, & \text { in } \Omega_{i}, \\ 0, & \text { in } \Omega \backslash\left(\Omega_{i^{\prime}} \cup \Omega_{i}\right) .\end{cases}
$$

We may then introduce the global skeleton space

$$
\mathcal{S}(\Omega) \equiv \operatorname{span}\left\{\Psi_{p, k}, \quad 1 \leq k \leq \mathcal{N}_{p}^{\Gamma}, 1 \leq p \leq n^{\Gamma}\right\}
$$

of dimension

$$
n_{\mathrm{SC}} \equiv \sum_{p=1}^{n^{\Gamma}} \mathcal{N}_{p}^{\Gamma} .
$$

Note that $\mathcal{S}(\Omega)$ is a continuous space thanks to the port compatibility requirement (7). Also note that in the definition of $\mathcal{S}(\Omega)$ we include only the $n^{\Gamma} \leq n_{0}^{\Gamma}$ ports on which we do not impose Dirichlet boundary conditions (we assume without loss of generality that we enforce Dirichlet boundary conditions on global ports $\left.\Gamma_{n^{\Gamma}+1}, \ldots, \Gamma_{n_{0}^{\Gamma}}\right)$.

Given the bubble spaces and the coupling modes, we now first introduce, for $1 \leq i \leq I$, the source bubble $\hat{b}_{i}^{f ; h}\left(\mu_{i}\right) \in \hat{B}_{\mathcal{M}(i) ; 0}^{h}$, which satisfies

$$
\hat{a}_{\mathcal{M}(i)}\left(\hat{b}_{i}^{f ; h}\left(\mu_{i}\right), v ; \mu_{i}\right)=\hat{f}_{\mathcal{M}(i)}\left(v ; \mu_{i}\right), \quad \forall v \in \hat{B}_{\mathcal{M}(i) ; 0}^{h}
$$

we define $b_{i}^{f ; h}\left(\mu_{i}\right) \equiv \mathcal{T}_{i}^{\text {rot }}\left(\hat{b}_{i}^{f ; h}\left(\mu_{i}\right) \circ \mathcal{T}_{i}^{-1}\right)$. Note that $b_{i}^{f ; h}(\mu)$ is a component-local particular solution to our global equation. We next introduce fundamental solutions $\hat{\phi}_{i, j, k}\left(\mu_{i}\right) \equiv$ $\hat{b}_{i, j, k}^{h}\left(\mu_{i}\right)+\hat{\psi}_{i, j, k}$ associated with each coupling mode $\hat{\psi}_{i, j, k}$ and bubble $\hat{b}_{i, j, k}^{h}\left(\mu_{i}\right) \in B_{\mathcal{M}(i) ; 0}^{h}$ such that $\hat{\phi}_{i, j, k}\left(\mu_{i}\right)$ satisfies

$$
\hat{a}_{\mathcal{M}(i)}\left(\hat{\phi}_{i, j, k}^{h}\left(\mu_{i}\right), v ; \mu_{i}\right)=0, \quad \forall v \in \hat{B}_{\mathcal{M}(i) ; 0}^{h}
$$


(note (19) is an equation for $\hat{b}_{i, j, k}^{h}\left(\mu_{i}\right)$ given the known $\left.\hat{\psi}_{i, j, k}\right)$; we define, on instantiated component $i, 1 \leq i \leq I, \phi_{i, j, k}\left(\mu_{i}\right) \equiv \mathcal{T}_{i}^{\mathrm{rot}}\left(\hat{\phi}_{i, j, k}\left(\mu_{i}\right) \circ \mathcal{T}_{i}^{-1}\right)$ and we introduce the global functions $\Phi_{p, k}(\mu) \in X^{h}(\Omega)$ such that, for $\pi_{p}=\left\{(i, j),\left(i^{\prime}, j^{\prime}\right)\right\}$,

$$
\Phi_{p, k}(\mu) \equiv \begin{cases}\phi_{i^{\prime}, j, k}\left(\mu_{i^{\prime}}\right), & \text { in } \Omega_{i^{\prime}}, \\ \phi_{i, j, k}\left(\mu_{i}\right), & \text { in } \Omega_{i}, \\ 0, & \text { in } \Omega \backslash\left(\Omega_{i^{\prime}} \cup \Omega_{i}\right) .\end{cases}
$$

Note that each $\Phi_{\mathcal{G}_{j}(i), k}(\mu)$ is the fundamental solution (local to a component pair) of our (homogeneous) global equation associated with the particular port mode $\chi_{i, j, k}$. Also note that $\Phi_{\mathcal{G}_{j}(i), k}(\mu)$ and $b_{i}^{f ; h}\left(\mu_{i}\right)$ scale linearly with certain "free" parameters, such as component-wide thermal conductivity or Young's modulus, which enter outside the bilinear form in (18) and (19); this will have important cost-saving implications in the context of RB approximation.

For each instantiated component we introduce a global function $u_{i}^{h}\left(\mu_{i}\right) \in X^{h}(\Omega)$ which represents the local solution on component $i$ in terms of the source bubbles $b_{i}^{f ; h}\left(\mu_{i}\right)$ and the fundamental solutions $\phi_{i, j, k}\left(\mu_{i}\right)$ as

$$
u_{i}^{h}\left(\mu_{i}\right)= \begin{cases}\sum_{i=1}^{I} b_{i}^{f ; h}\left(\mu_{i}\right)+\sum_{j=1}^{n_{\mathcal{M}(i)}^{\gamma}} \sum_{k=1}^{\mathcal{N}_{\mathcal{M}(i), j}^{\gamma}} \mathbb{U}_{\mathcal{G}_{i}(j), k}(\mu) \phi_{i, j, k}\left(\mu_{i}\right), & \text { in } \Omega_{i}, \\ 0, & \text { in } \Omega \backslash \Omega_{i},\end{cases}
$$

where the coefficient vector $\mathbb{U}_{\mathcal{G}_{i}(j), k}(\mu)$ contains global unknowns to be determined below. To couple the solutions in neighboring components we require weak flux continuity across global ports: ${ }^{\mathrm{d}}$ we write

$$
u^{h}(\mu)=\sum_{i=1}^{I} u_{i}^{h}\left(\mu_{i}\right)=\sum_{i=1}^{I} b_{i}^{f ; h}\left(\mu_{i}\right)+\sum_{p=1}^{n^{\Gamma}} \sum_{k=1}^{\mathcal{N}_{p}^{\Gamma}} \mathbb{U}_{p, k}(\mu) \Phi_{p, k}(\mu) ;
$$

we then test on all $v \in \mathcal{S}(\Omega)$ such that $u^{h}(\mu) \in X^{h}(\Omega)$ satisfies

$$
a\left(u^{h}(\mu), v ; \mu\right)=f(v ; \mu), \quad \forall v \in \mathcal{S}(\Omega) ;
$$

as before, our FE compliance output is $s^{h}(\mu)=f\left(u^{h}(\mu) ; \mu\right)$.

We emphasize that (23) is, thanks to Galerkin orthogonality of the fundamental solutions in (19) with respect to the associated bubble space, equivalent to (11). For this same reason we may further define an alternative skeleton space

$$
\mathcal{S}_{\text {symm }} \equiv \operatorname{span}\left\{\Phi_{p, k}(\mu), \quad 1 \leq k \leq \mathcal{N}_{p}^{\Gamma}, 1 \leq p \leq n^{\Gamma}\right\},
$$

such that $u^{h}(\mu) \in X^{h}(\Omega)$ satisfies

$$
a\left(u^{h}(\mu), v ; \mu\right)=f(v ; \mu), \quad \forall v \in \mathcal{S}_{\text {symm }}(\Omega) .
$$

There is no distinction between (23) and (25) in the FE static condensation context; however in the context of the SCRBE, direct approximation of (23) leads to a nonsymmetric Schur complement system, while direct approximation of (25) leads to a symmetric Schur complement system. In this paper we shall pursue the former with subsequent Schur complement symmetrization as the latter implies significantly larger online computational cost. 
The formulation (23) is equivalent to the symmetric linear-algebraic Schur complement system

$$
\mathbb{A}(\mu) \mathbb{U}(\mu)=\mathbb{F}(\mu)
$$

of size $n_{\mathrm{SC}}$, in which

$$
\begin{aligned}
\mathbb{A}_{(p, k),\left(p^{\prime}, k^{\prime}\right)}(\mu) & =a\left(\Phi_{p^{\prime}, k^{\prime}}(\mu), \Psi_{p, k} ; \mu\right), \\
\mathbb{F}_{(p, k)}(\mu) & =f\left(\Psi_{p, k} ; \mu\right)-\sum_{i=1}^{I} a\left(b_{i}^{f ; h}\left(\mu_{i}\right), \Psi_{p, k} ; \mu\right) .
\end{aligned}
$$

We may readily demonstrate the symmetry: by (19) and symmetry of $\hat{a}_{m}\left(\cdot, \cdot ; \mu_{i}\right)$ we obtain

$$
\begin{aligned}
\hat{a}_{\mathcal{M}(i)}\left(\hat{\phi}_{i, j, k}\left(\mu_{i}\right), \hat{\psi}_{i, j^{\prime}, k^{\prime}} ; \mu\right) & =\hat{a}_{\mathcal{M}(i)}\left(\hat{b}_{i, j, k}^{h}\left(\mu_{i}\right)+\hat{\psi}_{i, j, k}, \hat{b}_{i, j^{\prime}, k^{\prime}}^{h}\left(\mu_{i}\right)+\psi_{i, j^{\prime}, k^{\prime}} ; \mu_{i}\right) \\
& =\hat{a}_{\mathcal{M}(i)}\left(\hat{b}_{i, j^{\prime}, k^{\prime}}^{h}\left(\mu_{i}\right)+\hat{\psi}_{i, j^{\prime}, k^{\prime}}, \hat{b}_{i, j, k}^{h}\left(\mu_{i}\right)+\psi_{i, j, k} ; \mu_{i}\right) \\
& =\hat{a}_{\mathcal{M}(i)}\left(\hat{b}_{i, j^{\prime}, k^{\prime}}^{h}\left(\mu_{i}\right)+\hat{\psi}_{i, j^{\prime}, k^{\prime}}, \hat{\psi}_{i, j, k, k} ; \mu_{i}\right) \\
& =\hat{a}_{\mathcal{M}(i)}\left(\hat{\phi}_{i, j^{\prime}, k^{\prime}}\left(\mu_{i}\right), \hat{\psi}_{i, j, k} ; \mu_{i}\right),
\end{aligned}
$$

and as a result

$$
a\left(\Phi_{p^{\prime}, k^{\prime}}(\mu), \Psi_{p, k} ; \mu\right)=a\left(\Phi_{p, k}(\mu), \Psi_{p^{\prime}, k^{\prime}} ; \mu\right) .
$$

The matrix $\mathbb{A}(\mu)$ is thus symmetric and in particular may be rewritten as

$$
\mathbb{A}_{(p, k),\left(p^{\prime}, k^{\prime}\right)}(\mu)=\frac{1}{2} a\left(\Phi_{p^{\prime}, k^{\prime}}(\mu), \Psi_{p, k} ; \mu\right)+\frac{1}{2} a\left(\Phi_{p, k}(\mu), \Psi_{p^{\prime}, k^{\prime}} ; \mu\right)
$$

We shall invoke the interpretation (34) of $\mathbb{A}(\mu)$ to symmetrize the SCRBE Schur complement system below.

\section{Port compatibility}

The port compatibility requirement (7) between port basis functions associated with ports which may interconnect in a system - port of the same type - ensures solution continuity across shared global ports. We recall the archetype port basis functions $\hat{\chi}_{\mathcal{M}(i), j, k}$ introduced in (3), and we recall the associated physical (instantiated) port space basis functions $\chi_{i, j, k}$ introduced in (6). To honor (7), it is clear that the basis functions $\hat{\chi}_{m, j, k}$ on different archetype ports of the same port type must be defined differently according to the archetype port orientation.

To render this more precise we introduce for each unique port type a reference port domain $\beta \subset \mathbb{R}^{d-1}$; we assume for simplicity of exposition that there is only a single port type and thus $\beta$ needs no subscript. We then consider, on archetype component $m$, each archetype port domain $\hat{\gamma}_{m, j}$ as the image of $\beta$ under a rigid-body map $\mathcal{R}_{m, j}=\mathcal{R}_{m, j}^{\text {rot }} \mathcal{R}_{m, j}^{\text {tran }}$, where $\mathcal{R}_{m, j}^{\text {rot }}$ corresponds to rotation and $\mathcal{R}_{m, j}^{\mathrm{tran}}$ corresponds to translation, such that

$$
\hat{\gamma}_{m, j}=\mathcal{R}_{m, j}(\beta) ;
$$

this map is the key to honor the port compatibility requirement (7). ${ }^{\mathrm{e}}$

We then introduce, on the reference port domain $\beta$, a set of reference port modes $\hat{\chi}_{k}^{\beta}$, $1 \leq k \leq \mathcal{N}^{\beta}$, and an associated reference port space

$$
P^{\beta}=\operatorname{span}\left\{\hat{\chi}_{k}^{\beta}, \quad 1 \leq k \leq \mathcal{N}^{\beta}\right\}
$$


of dimension $\mathcal{N}^{\beta}$. We then define, on archetype port domain $\hat{\gamma}_{m, j}$ of type $\beta$, the archetype port space basis functions $\hat{\chi}_{m, j, k}, 1 \leq k \leq \mathcal{N}_{m}^{\gamma}, 1 \leq j \leq n_{m}^{\gamma}$, as

$$
\hat{\chi}_{m, j, k} \equiv \mathcal{R}_{m, j}^{\mathrm{rot}}\left(\hat{\chi}_{k}^{\beta} \circ \mathcal{R}_{m, j}^{-1}\right),
$$

Note that $\mathcal{N}_{m}^{\gamma}=\mathcal{N}^{\beta}$.

We now consider two instantiated port domains

$$
\gamma_{i, j}=\mathcal{T}_{i}\left(\hat{\gamma}_{\mathcal{M}(i), j}\right), \quad \gamma_{i^{\prime}, j^{\prime}}=\mathcal{T}_{i^{\prime}}\left(\hat{\gamma}_{\mathcal{M}\left(i^{\prime}\right), j^{\prime}}\right),
$$

on different instantiated components $i$ and $i^{\prime}$. From (5) we have for the associated port space basis functions

$$
\begin{aligned}
& \chi_{i, j, k}=\mathcal{T}_{i}{ }^{\mathrm{rot}}\left(\hat{\chi}_{\mathcal{M}(i), j, k} \circ \mathcal{T}_{i}^{-1}\right), \\
& \chi_{i^{\prime}, j v, k}=\mathcal{T}_{i^{\prime}}^{\text {rot }}\left(\hat{\chi}_{\mathcal{M}\left(i^{\prime}\right), j^{\prime}, k} \circ \mathcal{T}_{i^{\prime}}^{-1}\right) \text {, }
\end{aligned}
$$

and so, with (37),

$$
\begin{aligned}
\chi_{i, j, k} & =\mathcal{T}_{i}^{\mathrm{rot}}\left(\mathcal{R}_{\mathcal{M}(i), j}^{\mathrm{rot}}\left(\hat{\chi}_{k}^{\beta} \circ \mathcal{R}_{\mathcal{M}(i), j}^{-1}\right) \circ \mathcal{T}_{i}^{-1}\right), \\
\chi_{i^{\prime}, j^{\prime}, k} & =\mathcal{T}_{i^{\prime}}^{\mathrm{rot}}\left(\mathcal{R}_{\mathcal{M}\left(i^{\prime}\right), j^{\prime}}^{\mathrm{rot}}\left(\hat{\chi}_{k}^{\beta} \circ \mathcal{R}_{\mathcal{M}\left(i^{\prime}\right), j^{\prime}}^{-1}\right) \circ \mathcal{T}_{i^{\prime}}^{-1}\right) .
\end{aligned}
$$

Now, suppose that $\pi_{p}=\left\{(i, j),\left(i^{\prime}, j^{\prime}\right)\right\}$ for a shared global port $\Gamma_{p}$ such that $\gamma_{i, j}=\gamma_{i^{\prime}, j^{\prime}}$. In this case, from (35) and (38), we obtain

$$
\mathcal{T}_{i}\left(\mathcal{R}_{\mathcal{M}(i), j}(\beta)\right)=\mathcal{T}_{i^{\prime}}\left(\mathcal{R}_{\mathcal{M}\left(i^{\prime}\right), j^{\prime}}(\beta)\right) .
$$

We recall that $\mathcal{T}_{i}^{\text {def }}$ (for $1 \leq i \leq I$ ) when applied to a port corresponds to pure translation. As a result, application of the port mapping $\mathcal{T}_{i} \mathcal{R}_{\mathcal{M}(i), j}$ corresponds only to translation and rotation. We now recall that the rotation applied to $\beta$ on each side of (43) is unique, and we may thus conclude from (43) that $\mathcal{T}_{i}^{\text {rot }} \mathcal{R}_{\mathcal{M}(i), j}^{\text {rot }}=\mathcal{T}_{i^{\prime}}^{\text {rot }} \mathcal{R}_{\mathcal{M}\left(i^{\prime}\right), j^{\prime}}^{\text {rot }}$ With (41) and (42), we then obtain $\chi_{i, j, k}=\chi_{i^{\prime}, j^{\prime}, k}$, and we thus honor our port compatibility requirement (7).

\section{Model reduction}

The computational efficacy of our port-reduced SCRBE approach is realized through two separate model reduction techniques. As in the standard SCRBE approach [5] we consider component-interior model reduction through RB approximation [6] of the source bubbles (18) and of the fundamental solutions (19) to reduce the cost of each of the many component-interior linear solves required to form the Schur complement system. In addition to RB approximation in the component interiors, we employ port reduction [12] with empirical port modes to reduce the number of degrees of freedom on the ports and thus the size of the Schur complement system. We now discuss each of these techniques in more detail.

\section{Component-interior reduction}

For the component-interior model reduction we employ RB approximations

$$
\begin{aligned}
\tilde{b}_{i}^{f}\left(\mu_{i}\right) & \approx b_{i}^{f ; h}\left(\mu_{i}\right), \\
\tilde{\phi}_{i, j, k}\left(\mu_{i}\right) & \approx \phi_{i, j, k}^{h}\left(\mu_{i}\right),
\end{aligned}
$$

and thus $\tilde{\Phi}_{p, k}(\mu) \approx \Phi_{p, k}(\mu)$. The purpose of these RB approximations is to allow for efficient formation of an approximation to the Schur complement system (54): each RB approximation $\tilde{b}_{i}^{f}\left(\mu_{i}\right)$ or $\tilde{\phi}_{i, j, k}\left(\mu_{i}\right)$ is associated with a rapidly convergent [7] RB space specifically tailored to the particular bubble and to the parameter dependence defined by 
the corresponding (archetype domain) PDE (18) or (19). All RB bubble spaces are thus different, and furthermore each space is typically of much lower dimension than the original FE spaces $\hat{B}_{m ; 0}^{h}$. As a consequence, the RB approximations to the solutions of (18) and (19) are obtained at significantly reduced computational cost with minimal compromise to solution accuracy. The RB method is now considered standard, and we refer the reader to [6] for all technical details relevant to the particular class of problems (linear elliptic) that we consider here.

We now introduce the SCRBE approximation $\tilde{u}^{*}(\mu) \approx u^{h}(\mu)$ as

$$
\tilde{u}^{*}(\mu)=\sum_{i=1}^{I} \tilde{b}_{i}^{f}\left(\mu_{i}\right)+\sum_{p=1}^{n^{\Gamma}} \sum_{k=1}^{\mathcal{N}_{p}^{\Gamma}} \tilde{\mathbb{U}}_{p, k}^{*}(\mu) \tilde{\Phi}_{p, k}(\mu),
$$

and we again choose $\mathcal{S}(\Omega)$ as the test space such that $\tilde{u}^{*}(\mu) \in X^{h}(\Omega)$ satisfies

$$
a\left(\tilde{u}^{*}(\mu), v ; \mu\right)=f(v ; \mu), \quad \forall v \in \mathcal{S}(\Omega) ;
$$

the equivalent linear-algebraic system is

$$
\tilde{\mathbb{A}}^{*}(\mu) \tilde{\mathbb{U}}^{*}(\mu)=\tilde{\mathbb{F}}(\mu)
$$

where

$$
\begin{aligned}
\tilde{\mathbb{A}}_{(p, k),\left(p^{\prime}, k^{\prime}\right)}^{*}(\mu) & =a\left(\tilde{\Phi}_{p^{\prime}, k^{\prime}}(\mu), \tilde{\Psi}_{p, k} ; \mu\right), \\
\tilde{\mathbb{F}}_{(p, k)}(\mu) & =f\left(\tilde{\Psi}_{p, k} ; \mu\right)-\sum_{i=1}^{I} a\left(b_{i}^{f ; h}\left(\mu_{i}\right), \tilde{\Psi}_{p, k} ; \mu\right),
\end{aligned}
$$

for $1 \leq k \leq \mathcal{N}_{p}^{\Gamma}, 1 \leq k \leq \mathcal{N}_{p^{\prime}}^{\Gamma}, 1 \leq p, p^{\prime} \leq n^{\Gamma}$. Note that $\tilde{\mathbb{A}}^{*}(\mu)$ in (49) is non-symmetric because each RB approximation $\tilde{\phi}_{i, j, k}\left(\mu_{i}\right)$ (mapped to the respective archetype domain) satisfies (19) only with respect to the associated RB bubble subspace. These RB approximations are thus not Galerkin orthogonal with respect to other bubble spaces; recall that this Galerkin orthogonality (together with symmetry of $\hat{a}_{m}\left(\cdot, \cdot ; \mu_{i}\right)$ ) is the key to the symmetry of $\mathbb{A}(\mu)$ as demonstrated in (29). To recover symmetry we have two options: we may either, as in $[5,12]$, test on a space

$$
\tilde{\mathcal{S}}_{\text {symm }}(\Omega)=\operatorname{span}\left\{\tilde{\Phi}_{p, k}(\mu), \quad 1 \leq k \leq \mathcal{N}_{p}^{\Gamma}, 1 \leq p \leq n^{\Gamma}\right\}
$$

in (47), or we may explicitly symmetrize $\tilde{\mathbb{A}}^{*}(\mu)$ by algebraic manipulation. The former option necessitates larger offline and online computational cost and storage, in fact, when compared to the latter, by a multiplicative factor equal to the number of RB basis functions.

We thus elect to recover symmetry by algebraic manipulation: we exploit the interpretation (34) of $\mathbb{A}(\mu)$ and we define $\tilde{\mathbb{A}}(\mu) \approx \mathbb{A}(\mu)$ as

$$
\tilde{\mathbb{A}}(\mu)=\frac{1}{2} \tilde{\mathbb{A}}^{*}(\mu)+\frac{1}{2} \tilde{\mathbb{A}}^{*}(\mu)^{\mathrm{T}},
$$

such that

$$
\tilde{\mathbb{A}}_{(p, k),\left(p^{\prime}, k^{\prime}\right)}(\mu)=\frac{1}{2} a\left(\tilde{\Phi}_{p^{\prime}, k^{\prime}}(\mu), \Psi_{p, k} ; \mu\right)+\frac{1}{2} a\left(\tilde{\Phi}_{p, k}(\mu), \Psi_{p^{\prime}, k^{\prime}} ; \mu\right) .
$$

We may then finally introduce our symmetric SCRBE linear-algebraic system as

$$
\tilde{\mathbb{A}}(\mu) \tilde{\mathbb{U}}(\mu)=\tilde{\mathbb{F}}(\mu),
$$


and we define the SCRBE field approximation $\tilde{u}(\mu) \approx u^{h}(\mu)$ as

$$
\tilde{u}(\mu)=\sum_{i=1}^{I} \tilde{b}_{i}^{f}\left(\mu_{i}\right)+\sum_{p=1}^{n^{\Gamma}} \sum_{k=1}^{\mathcal{N}_{p}^{\Gamma}} \tilde{\mathbb{U}}_{p, k}(\mu) \tilde{\Phi}_{p, k}(\mu) .
$$

The associated SCRBE compliance output approximation is $\tilde{s}(\mu)=f(\tilde{u}(\mu) ; \mu)$.

Note that in actual practice, we assemble (54) through a direct-stiffness procedure from component-local matrix and vector blocks associated with and assembled for each of the I component instantiations; the procedure is described in detail in [5,12]. The assembly of these component-local quantities constitutes the majority of online computational cost. However, we need only perform the assembly for each unique component instantiation, as identical (or "cloned") components may share local matrices and vectors. We thus realize significant computational savings for systems which consist of instantiations of many component clones, such that we need only consider $I_{\mathrm{eff}} \ll I$ effective component instantiations for this assembly proceedure.

There are two particularly important situations in which different component instantiations are effectively clones in the sense that the component-local matrix and vector blocks may still be re-used: First, matrix and vector blocks computed for component instantiations which differ only in spatial orientation are (in the case that material properties do not depend on spatial orientation, such as in isotropic linear elasticity) identical thanks to cancellation of the mapping Jacobians in the archetype domain bilinear form; second, "free" parameters such as component-wide thermal conductivity or Young's modulus enter outside the bilinear forms in (18) and (19), and thus the associated matrix blocks will only differ by a scaling factor. As a result, we often obtain $I_{\text {eff }} \ll I$ in practice. We discuss this situation further under "Computational procedures" later in this section.

\section{Port reduction}

\section{Framework}

While the RB approximation is concerned with component-interior model reduction, we apply port reduction to reduce the number of degrees of freedom associated with component interfaces. For the port reduction procedure we shall consider on each global port $\Gamma_{p}$ only $n_{\mathrm{A}, p}^{\Gamma} \leq \mathcal{N}_{p}^{\Gamma}$ port modes as "Active" and thus contributing to the approximation; for substantial computational savings we require $n_{\mathrm{A}, p}^{\Gamma} \ll \mathcal{N}_{p}^{\Gamma}$. We consider in this subsection the generic port reduction framework and in the next subsection our particular choice of port space basis functions which realizes $n_{\mathrm{A}, p}^{\Gamma} \ll \mathcal{N}_{p}^{\Gamma}$.

Based on the $n_{\mathrm{A}, p}^{\Gamma}$ active modes associated with each global port we introduce a portreduced skeleton space $\mathcal{S}^{\mathrm{PR}}(\Omega) \subseteq \mathcal{S}(\Omega)$ as

$$
\mathcal{S}^{\mathrm{PR}}(\Omega) \equiv \operatorname{span}\left\{\Psi_{p, k}, \quad 1 \leq k \leq n_{\mathrm{A}, p}^{\Gamma}, 1 \leq p \leq n^{\Gamma}\right\}
$$

of dimension

$$
n_{\mathrm{A}} \equiv \sum_{p=1}^{n^{\Gamma}} n_{\mathrm{A}, p}^{\Gamma} \leq n_{\mathrm{SC}}
$$

We further introduce a port-reduced approximation $\tilde{u}^{\mathrm{PR}, *}(\mu) \approx u^{h}(\mu)$ as

$$
\tilde{u}^{\mathrm{PR}, *}(\mu)=\sum_{i=1}^{I} \tilde{b}_{i}^{f}\left(\mu_{i}\right)+\sum_{p=1}^{n^{\Gamma}} \sum_{k=1}^{n_{\mathrm{A}, p}^{\Gamma}} \tilde{\mathbb{U}}_{p, k}^{*}(\mu) \tilde{\Phi}_{p, k}(\mu) .
$$


We now choose $\mathcal{S}^{\mathrm{PR}}(\Omega)$ as our test space such that

$$
a\left(\tilde{u}^{\mathrm{PR}, *}(\mu), v ; \mu\right)=f(v ; \mu), \quad \forall v \in \mathcal{S}^{\mathrm{PR}}(\Omega),
$$

which leads to the linear-algebraic system

$$
\tilde{\mathbb{A}}^{\mathrm{PR}, *}(\mu) \tilde{\mathbb{U}}^{\mathrm{PR}, *}(\mu)=\tilde{\mathbb{F}}^{\mathrm{PR}}(\mu)
$$

of size $n_{\mathrm{A}}$, where

$$
\begin{aligned}
\tilde{\mathbb{A}}_{(p, k),\left(p^{\prime}, k^{\prime}\right)}^{\mathrm{PR}, *}(\mu) & =a\left(\tilde{\Phi}_{p^{\prime}, k^{\prime}}(\mu), \Psi_{p, k} ; \mu\right), \\
\tilde{\mathbb{F}}_{(p, k)}^{\mathrm{PR}}(\mu) & =f\left(\Psi_{p, k} ; \mu\right)-\sum_{i} a\left(b_{i}^{f ; h}\left(\mu_{i}\right), \Psi_{p, k} ; \mu\right),
\end{aligned}
$$

for $1 \leq k \leq n_{\mathrm{A}, p}^{\Gamma}, 1 \leq k^{\prime} \leq n_{\mathrm{A}, p^{\prime}}^{\Gamma}, 1 \leq p, p^{\prime} \leq n^{\Gamma}$. We then symmetrize as

$$
\tilde{\mathbb{A}}^{\mathrm{PR}} \equiv \frac{1}{2} \tilde{\mathbb{A}}^{\mathrm{PR}, *}(\mu)+\frac{1}{2} \tilde{\mathbb{A}}^{\mathrm{PR}, *}(\mu)^{\mathrm{T}},
$$

we define the port-reduced SCRBE system as

$$
\tilde{\mathbb{A}}^{\mathrm{PR}}(\mu) \tilde{\mathbb{U}}^{\mathrm{PR}}(\mu)=\tilde{\mathbb{F}}^{\mathrm{PR}}(\mu),
$$

and we define the port-reduced SCRBE field approximation $\tilde{u}^{\mathrm{PR}}(\mu) \approx u^{h}(\mu)$ as

$$
\tilde{u}^{\mathrm{PR}}(\mu)=\sum_{i=1}^{I} \tilde{b}_{i}^{f}\left(\mu_{i}\right)+\sum_{p=1}^{n^{\Gamma}} \sum_{k=1}^{n_{\mathrm{A}, p}^{\Gamma}} \tilde{\mathbb{U}}_{p, k}^{\mathrm{PR}}(\mu) \tilde{\Phi}_{p, k}(\mu) .
$$

The associated port-reduced SCRBE compliance output approximation is $\tilde{s}^{\mathrm{PR}}(\mu)=$ $f\left(\tilde{u}^{\mathrm{PR}}(\mu) ; \mu\right)$.

The purpose of port reduction is of course to reduce the size of the Schur complement system - and thus computational cost - while maintaining accuracy of the approximation. The size of the system (64), $n_{\mathrm{A}}$, is equal to the total number of active port modes in the system. In practice, we shall typically invoke only a few port degrees of freedom on each port such that $n_{\mathrm{A}} \ll n_{\mathrm{SC}}$. A good choice for the port modes $\chi_{i, j, k}$ is key to the accuracy of the port-reduced SCRBE approximation, and is the focus of the next subsection.

\section{Empirical port mode training}

To ensure port compatibility we must for each port type develop our port basis on the associated reference port domain $\beta$ as discussed under "Port compatibility" above. To this end we pursue a pairwise training algorithm that provides a port space tailored to the family of solutions associated with this port type. We shall develop bases for the full port spaces (6) and not merely the space spanned by "Active" modes; the remaining "Inactive" modes shall play a role in certification (for residual calculation), which we discuss further in the "Certification framework" section.

Our port spaces shall consist of three sets of modes. The first set of modes is explicitly specified and consists of the six modes associated with rigid-body motion. ${ }^{f}$ We include these six modes for two reasons: first, it simplifies the procedure for specification of typical Dirichlet boundary conditions, and second, it ensures invertibility of the Schur complement operator associated with "Inactive" modes, which is a property we require for our non-conforming error estimation framework. 
The second set of modes consists of the $n_{\text {pod }}^{\beta} \leq \mathcal{N}^{\beta}-6$ modes which shall be the outcome of our pairwise training algorithm. The third set of modes consists of $\mathcal{N}^{\beta}-$ $n_{\text {pod }}^{\beta}-6$ singular Sturm-Liouville eigenmodes restricted to the orthogonal complement of the first $n_{\text {pod }}^{\beta}+6$ empirical modes [12]. These modes serve to complete the discrete port space in a numerically stable fashion.

Recall that the total number of modes associated with the reference port $\beta$ is $\mathcal{N}^{\beta}$. We consider here the case $d=3$ and thus $\beta \subset \mathbb{R}^{2}$; each port mode $\hat{\chi}_{i}^{\beta}, 1 \leq i \leq \mathcal{N}^{\beta}$, has the form $\hat{\chi}_{i}^{\beta}=\left(\hat{\chi}_{i}^{\beta, 1}, \hat{\chi}_{i}^{\beta, 2}, \hat{\chi}_{i}^{\beta, 3}\right)$, where the number of degrees of freedom associated with each field component $\hat{\chi}_{i}^{\beta, j}$ is $\mathcal{N}^{\beta} / 3$.

In the case that $\beta$ is the square $\beta=[-0.5,0.5]^{2}$, the first six reference port modes are explicitly defined as

$$
\hat{\chi}_{1}^{\beta}=(1,0,0), \quad \hat{\chi}_{2}^{\beta}=(0,1,0), \quad \hat{\chi}_{3}^{\beta}=(0,0,1),
$$

for the three ports associated with translation; as

$$
\hat{\chi}_{4}^{\beta}(\xi, \eta)=(-\eta, \xi, 0)
$$

for the mode associated with pure rotation; and as

$$
\hat{\chi}_{5}^{\beta}(\xi, \eta)=(0,0, \xi), \quad \hat{\chi}_{6}^{\beta}=(0,0, \eta),
$$

for the two modes associated with flipping. Note these six modes are mutually $\left(L^{2}(\beta)\right)^{d}$ orthonormal. (If $\beta$ is not the square $\beta=[-0.5,0.5]^{2}$ we apply Gram-Schmidt orthonormalization to these first six modes to recover $\left(L^{2}(\beta)\right)^{d}$-orthonormality.)

The next $n_{\text {pod }}^{\beta}$ port modes are the outcome of our pairwise empirical training algorithm. In this algorithm we exploit the fact that within any system, the solution on any global (shared, say) port is determined completely by the parameter values assigned to the pair of components sharing the port and the (typically relatively smooth) solution on all other ports associated with these two components. The purpose of our pairwise training algorithm is to explore the associated "solution manifold" induced by local parameter dependence and neighboring ports in a systematic fashion such that the empirical modes associated with each port type are tailored to all possible component connectivity and all admissible component parameter values.

For our empirical training algorithm we shall require discrete "Legendre polynomials" $L_{i}^{\beta}, 1 \leq i \leq \mathcal{N}^{\beta} / 3$, such that the $L_{i}^{\beta}$ are the eigenvectors of a scalar singular Sturm-Liouville eigenproblem [16] over $\beta$ ordered according to increasing eigenvalue; we shall also require a univariate random variable $r$ with uniform density; and we introduce an algorithm tuning parameter $\gamma>1$ related to anticipated regularity. We then identify one or several pairs of components in the component library that may connect through a global port of the relevant port type $\beta$.

The empirical training procedure for each such pair is now given by Algorithm 1: we sample (solve) each pair $N_{\text {sample }}$ times for different (random) parameters and different (random but smooth thanks to the parameter $\gamma>1$ ) boundary conditions on all nonconnected ports (note that we assign random boundary conditions independently to each vector component); for each such sample we extract the solution on the shared port $\Gamma_{p^{*}}$ of the relevant type, map it to the reference port $\beta$, subtract from this mapped solution its orthogonal $\left(L^{2}(\beta)\right)^{d}$-projection onto each of the six rigid body modes $\hat{\chi}_{i}^{\beta}, 1 \leq i \leq 6$, and 
then finally include the result $\zeta$ in a snapshot set $S_{\text {pair }}$ associated with the current pair. Note that in Algorithm $1(\cdot, \cdot)_{L^{2}(\beta)}$ refers to the vector $\left(L^{2}(\beta)\right)^{d}$ inner product.

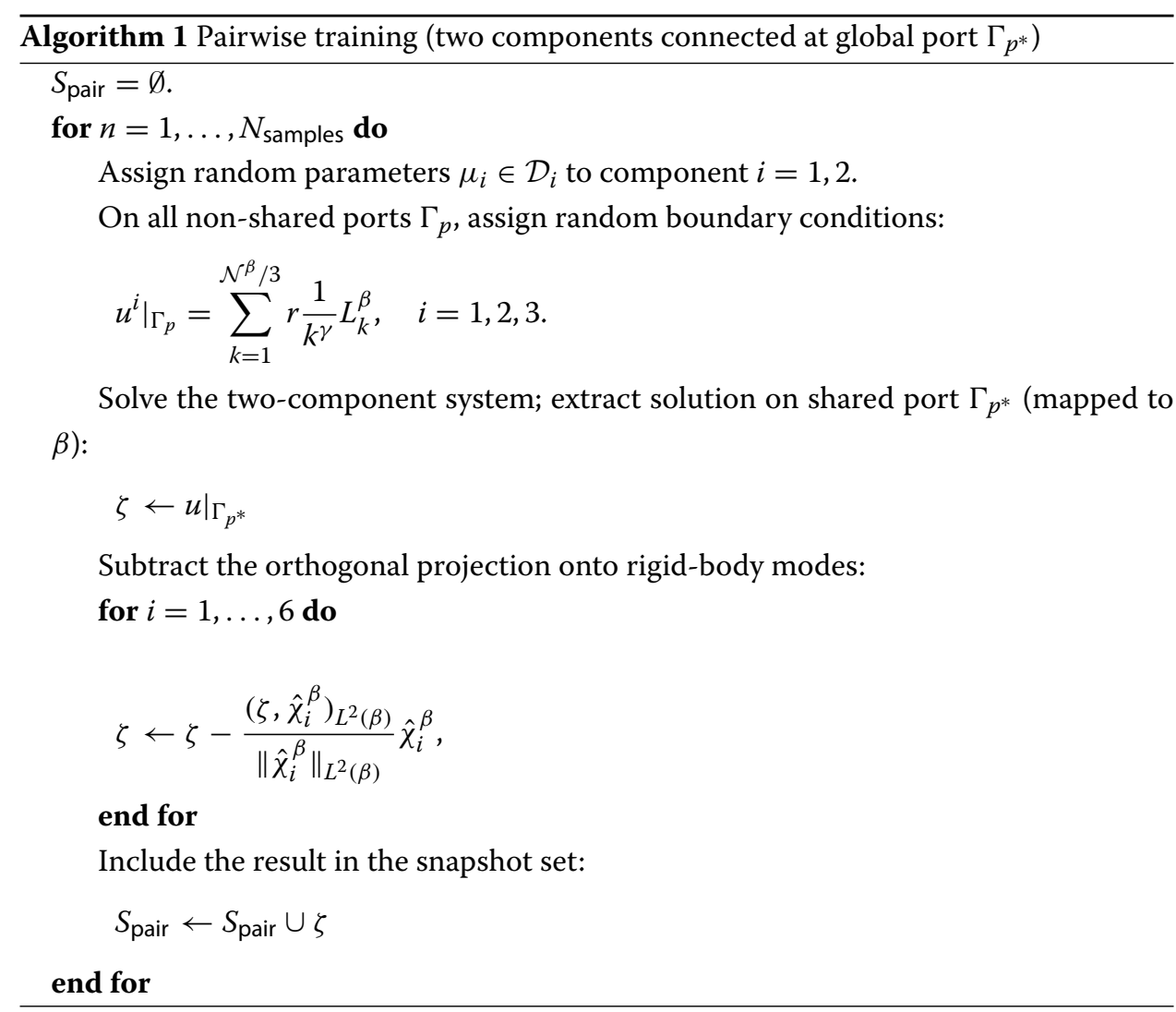

After pairwise training of all pairs relevant for one port type, we form the bigger snapshot set

$$
S_{\text {type }}=\bigcup_{\text {pair }} S_{\text {pair }}
$$

We then perform a data compression step: we invoke the proper orthogonal decomposition (POD) [17] (with respect to the vector $\left(L^{2}(\beta)\right)^{d}$ inner product). The output from the POD procedure is a set of $n_{\text {pod }}^{\beta}$ mutually $\left(L^{2}(\beta)\right)^{d}$-orthonormal POD modes which are also orthonormal to the six first modes $\hat{\chi}_{i}^{\beta}, 1 \leq i \leq 6$, related to rigid-body motion. We choose these $n_{\text {pod }}^{\beta}$ POD modes as our next reference port basis functions $\hat{\chi}_{6+i}^{\beta}, 1 \leq i \leq n_{\text {pod }}^{\beta}$; we typically observe rapid (often exponential) convergence [12] of these POD modes with respect to the input snapshot set $S_{\text {type }}$.

We refer to all first $n_{\text {pod }}^{\beta}+6$ port modes as our empirical port modes. If $n_{\text {pod }}^{\beta}$ is chosen such that $n_{\text {pod }}^{\beta}+6<\mathcal{N}^{\beta}$, we now complete the discrete space with Sturm-Liouville singular eigenmodes restricted to the orthogonal complement $\operatorname{space}\left(\operatorname{span}\left\{\hat{\chi}_{i}^{\beta}\right\}_{i=1}^{n_{\text {pod }}^{\beta}+6}\right)^{\perp}$ (of dimension $\mathcal{N}^{\beta}-n_{\text {pod }}^{\beta}-6$ ) as discussed in detail in [12].

We finally note that for our pairwise training approach we may employ the (non-portreduced) SCRBE framework or we may use standard FE approximations. The computational cost associated with empirical training is not critical as the procedure is performed 
offline. For our numerical results in this paper we have used the non-port-reduced SCRBE framework to calculate empirical modes.

\section{Computational procedures}

The computational procedures associated with our port-reduced SCRBE approximation framework naturally decouple into an offline preprosessing stage and an online evaluation stage, and we now discuss each in more detail. Note we provide here only descriptions of each of the offline and online steps involved; for detailed online operation counts we refer to [12].

\section{Offline}

The offline stage is the preprosessing stage - performed only once - in which we construct and prepare the archetype component library. This stage consists of the following steps.

Off1. Empirical pairwise training by Algorithm 1. For each port type we sample pairs of components to obtain efficient port space basis functions $\hat{\chi}_{k}^{\beta}, 1 \leq k \leq \mathcal{N}^{\beta}$, associated with each reference port domain $\beta$. In the current implementation, we employ the non-port-reduced SCRBE [5] (rather than standard global FE) for the pairwise training.

Off2. RB space construction. For each archetype component $m, 1 \leq m \leq M$, we must train $\sum_{j=1}^{n_{m}^{\gamma}} \mathcal{N}_{m, j}^{\gamma}+1$ different RB spaces to accommodate the RB approximations (44) and (45). Each construction of an RB space requires a number of component-local FE solves (each associated with an RB space basis function), and thus this step is potentially rather expensive, depending on the component spatial discretization and parametric complexity $\hat{Q}_{m}^{a}$ and $\hat{Q}_{m}^{f}$ in the bilinear and linear form expansions (1).

Note, however, that the construction of the RB approximation spaces (subsequent to port space construction) is embarrassingly parallel. Also note that we do not consider parameters for spatial orientation (because of the mapping Jacobian cancellations in the archetype domain formulation), and furthermore recall that components often have "free" parameters such as component-wide thermal conductivity or Young's modulus, with which the solutions to (18) and (19) simply scale linearly. As a result, RB space dimensions are typically rather small (around ten basis functions often suffice for each RB space), and thus although this step typically dominates offline cost the computational effort is not onerous: typically a couple of CPU hours is required for each archetype component.

Off3. Online dataset preparation. For each archetype component we construct data to enable efficient assembly of the component-local Schur complement matrix and vector blocks in the subsequent online stage. The computation time depends stongly on component spatial discretization and parametric complexity, but is typically between minutes and hours (on a single CPU) for each component. The online dataset also contains all RB basis functions, which are required for online global field visualization, if desired.

Off4. Data loading. We finally read the online datasets (typically a few hundred $\mathrm{Mb}$ ) for all library components into computer memory to prepare for the online stage. 
Note that with our current implementation of the framework, since we employ the nonport-reduced SCRBE in step Off1 above, we must first perform a step Off0a (similar to Off2) and then a step Off0b (similar to Off3) in order to enable the necessary "online" pair evaluation in Off1.

\section{Online}

The online stage is the stage in which we instantiate archetype components, and assemble and solve our system. This stage consists of the following steps, which in the current implementation is performed on a single CPU.

On1. Component instantiation. Instantiate I components from the library, assign the relevant parameter values to each component, and connect components to other components through ports of the same type to form a system; this step is most easily effected through a graphical user interface [Additional file 1].

On2. Schur complement system formation. Perform component-local RB solves (of small RB dimension) associated with all "Active" degrees of freedom to obtain (RB coefficients for) the RB approximations $\tilde{\phi}_{i, j^{\prime}, k^{\prime}}\left(\mu_{i}\right)$ and $\tilde{b}^{f}\left(\mu_{i}\right)$, assemble the associated matrix and vector blocks for each component, and assemble the Schur complement system (64) through a direct-stiffness procedure [5,12].

The entries in the component-local matrix blocks are of the form

$$
\tilde{\mathbb{A}}_{\mathrm{A}, \mathrm{A} ;(j, k),\left(j^{\prime}, k^{\prime}\right)}^{i}\left(\mu_{i}\right)=\frac{1}{2} a_{i}\left(\tilde{\phi}_{i, j^{\prime}, k^{\prime}}\left(\mu_{i}\right), \psi_{i, j, k} ; \mu_{i}\right)+\frac{1}{2} a_{i}\left(\tilde{\phi}_{i, j, k}\left(\mu_{i}\right), \psi_{i, j^{\prime}, k^{\prime}} ; \mu_{i}\right)
$$

(the symmetrization is performed on the component level) and the entries in the component-local vector blocks are of the form $\tilde{\mathbb{F}}_{\mathrm{A} ;(j, k)}^{i}\left(\mu_{i}\right)=f\left(\psi_{i, j, k} ; \mu\right)-$ $a_{i}\left(\tilde{b}^{f}\left(\mu_{i}\right), \psi_{i, j, k} ; \mu\right)$; the subscripts A refer to assembly of "Active" component matrices and vectors. However, thanks to an efficient construction-evaluation procedure [6], which relies on the affine operator expansions (1), only the RB coefficients associated with $\tilde{\phi}_{i, j^{\prime}, k^{\prime}}\left(\mu_{i}\right)$ and $\tilde{b}^{f}\left(\mu_{i}\right)$ are required for this assembly step. We emphasize in particular that the underlying component FE discretization is never invoked. We recall that parameters related to spatial orientation (component "docking") do not appear in the (archetype) bilinear forms due to cancellation of the associated Jacobians (we demonstrate this for isotropic linear elasticity in the "Microtruss beam application" section); and moreover, certain parametric variations such as component-wide conductivity or Young's modulus are "free" in the sense that they enter as scalars outside the bilinear forms in (18) and (19). As a consequence, matrix and vector blocks associated with different component instantiations are in practice often identical (in the context of "free" parameters up to a multiplicative constant). We may thus in typical systems often consider only $I_{\text {eff }} \ll I$ effectively different (or unique) component instantiations, for which we perform RB solves and assemble component-local matrices and vectors. The component-local matrices and vectors for the remaining $I-I_{\text {eff }}$ component instantiations are then simply copies of the respective data from effectively identical components. This consideration of component "clones" together with 
the realization of "docking" parameter cancellation and "free" parameters contribute significantly to the modest computational cost associated with On2.g The typical computation time is a few seconds.

On3. Evaluate. Solve the "Active" Schur complement system, and evaluate any relevant derived quantities from the solution vector (for example a compliance output). The typical computation time is a few seconds.

The computational cost associated with this online stage is dominated by On 2 (when $I_{\text {eff }}$ is close to $I$ ) or On3 (when $I_{\text {eff }} \ll I$ ). However, the offline and online stages above are only concerned with the port-reduced SCRBE approximation. We consider the computational procedures associated with a posteriori error estimation in the next section.

\section{Certification framework}

Our port-reduced SCRBE approximation is equipped with efficiently computable $a$ posteriori error bounds and estimators that provide certificates for the error in the approximation with respect to the underlying global FE discretization. We employ in this paper the energy-norm and compliance output bound developed in [12], and we present the main ingredients and certain extensions below. We furthermore sharpen the bounds by consideration of a multi-reference parameter bound conditioner.

The error in our approximation derives from two sources: port reduction and RB approximation. Below we first address the error due to port reduction, that is to say, the case in which the error due to RB approximation is zero. In this case the error bound presentation simplifies significantly and in particular admits a pure functional interpretation. We then subsequently perturb the equivalent algebraic interpretation to provide a bound for the general case in which the error due to RB approximation is non-zero.

\section{Port reduction error contribution}

We assume in this subsection only that the only source of error is port reduction and hence that there is no RB-induced error. We introduce the function

$$
u^{\mathrm{PR}}(\mu)=\sum_{i=1}^{I} b^{f ; h}\left(\mu_{i}\right)+\sum_{p=1}^{n^{\Gamma}} \sum_{k=1}^{n_{\mathrm{A}, p}^{\Gamma}} \mathbb{U}_{p, k}^{\mathrm{PR}}(\mu) \Phi_{p, k}(\mu) \in X^{h}(\Omega),
$$

which satisfies

$$
a\left(u^{\mathrm{PR}}(\mu), v ; \mu\right)=f(v ; \mu), \quad \forall v \in \mathcal{S}^{\mathrm{PR}}(\Omega) ;
$$

hence $u^{\mathrm{PR}}(\mu)$ is the port-reduced approximation to $u^{h}(\mu)$ obtained in the absence of RB errors. We note that we may (as in (25)) replace the skeleton space $\mathcal{S}^{\mathrm{PR}}(\Omega)$ in (72) by the skeleton space

$$
\mathcal{S}_{\text {symm }}^{\mathrm{PR}}(\Omega)=\operatorname{span}\left\{\Phi_{p, k}(\mu), \quad 1 \leq k \leq n_{\mathrm{A}, p}^{\Gamma}, 1 \leq p \leq n^{\Gamma}\right\} \subset \mathcal{S}_{\text {symm }}(\Omega),
$$

and thus $u^{\mathrm{PR}}(\mu) \in X^{h}(\Omega)$ also satisfies

$$
a\left(u^{\mathrm{PR}}(\mu), v ; \mu\right)=f(v ; \mu), \quad \forall v \in \mathcal{S}_{\text {symm }}^{\mathrm{PR}}(\Omega) ;
$$

note that $u^{\mathrm{PR}}(\mu) \notin \mathcal{S}_{\text {symm }}^{\mathrm{PR}}(\Omega)$ because of the source bubble terms $b^{f ; h}\left(\mu_{i}\right)$ in (71). 
We define the associated (RB-error-free) error field as

$$
\begin{aligned}
e_{0}^{h}(\mu) & \equiv u^{h}(\mu)-u^{\mathrm{PR}}(\mu) \\
& =\sum_{p=1}^{n^{\Gamma}}\left(\sum_{k=1}^{n_{\mathrm{A}, p}^{\Gamma}}\left(\mathbb{U}_{p, k}(\mu)-\mathbb{U}_{p, k}^{\mathrm{PR}}(\mu)\right) \Phi_{p, k}(\mu)+\sum_{k=n_{\mathrm{A}, p}^{\Gamma}+1}^{\mathcal{N}_{p}^{\Gamma}} \mathbb{U}_{p, k}(\mu) \Phi_{p, k}(\mu)\right),
\end{aligned}
$$

(in which the subscript ${ }_{0}$ refers to the case of zero RB error contribution) and we note that $e_{0}^{h}(\mu) \in \mathcal{S}_{\text {symm }}(\Omega)$ because the source bubble contributions from $u^{h}(\mu)$ and $u^{\mathrm{PR}}(\mu)$ cancel. Our goal is to develop a bound for the energy $\left\|e_{0}^{h}(\mu)\right\|_{\mu}$, where

$$
\|\cdot\|_{\mu} \equiv \sqrt{a(\cdot, \cdot ; \mu)}
$$

is the usual energy norm. From (25) and (74) we see that

$$
a\left(e_{0}^{h}(\mu), v ; \mu\right)=f(v)-a\left(u^{\mathrm{PR}}(\mu), v ; \mu\right), \quad \forall v \in \mathcal{S}_{\text {symm }}(\Omega) ;
$$

this error-residual relationship is the point of departure for our error bound development.

Thanks to coercivity and symmetry of $a(\cdot, \cdot ; \mu)$, the error field $e_{0}^{h}(\mu)$ admits the equivalent definition

$$
e_{0}^{h}(\mu)=\arg \min _{\nu \in \mathcal{S}_{\text {symm }}(\Omega)} \mathcal{J}(v ; \mu),
$$

where

$$
\mathcal{J}(v ; \mu) \equiv \frac{1}{2} a(v, v)-\left(f(v)-a\left(u^{\mathrm{PR}}(\mu), v ; \mu\right)\right),
$$

and furthermore $\left\|e_{0}^{h}(\mu)\right\|_{\mu}^{2}=a\left(e_{0}^{h}(\mu), e_{0}^{h}(\mu) ; \mu\right)=-2 \mathcal{J}\left(e_{0}^{h}(\mu) ; \mu\right)$. We now relax the minimization (78) by consideration of a discontinuous (non-conforming) skeleton space

$$
\begin{aligned}
& \mathcal{S}_{\text {symm }}^{\mathrm{NC}}(\Omega) \equiv \mathcal{S}_{\text {symm }}^{\mathrm{PR}}(\Omega) \\
& \oplus \operatorname{span}\left\{\phi_{i, j, k}(\mu), \quad\left(n_{\mathrm{A}, i, j}^{\gamma}+1\right) \leq k \leq \mathcal{N}_{i, j}^{\gamma}, 1 \leq j \leq n_{i}^{\gamma}, 1 \leq i \leq I\right\} \\
& \equiv \operatorname{span}\left\{\Phi_{i}^{\prime}(\mu), \quad 1 \leq i \leq n_{\mathrm{NC}}\right\},
\end{aligned}
$$

in which the basis functions $\Phi_{i}^{\prime}(\mu), 1 \leq i \leq n_{\mathrm{NC}}$, merely represent a re-indexing of the basis functions $\Phi_{p, k}(\mu), 1 \leq k \leq n_{p}^{\Gamma}, 1 \leq p \leq n^{\Gamma}$, and $\phi_{i, j, k}(\mu),\left(n_{\mathrm{A}, i, j}^{\gamma}+1\right) \leq k \leq \mathcal{N}_{i, j}^{\gamma}, 1 \leq$ $j \leq n_{i}^{\gamma}, 1 \leq i \leq I$. Note that the $\phi_{i, j, k}(\mu)$ represent independent (non-conforming) degrees of freedom local to component $i$. The dimension of $\mathcal{S}_{\text {symm }}^{\mathrm{NC}}(\Omega)$ is

$$
n_{\mathrm{NC}}=n_{\mathrm{A}}+\sum_{i=1}^{I} \sum_{j=1}^{n_{i}^{\gamma}} \mathcal{N}_{i, j}^{\gamma}-n_{\mathrm{A}, i, j}^{\gamma} \geq n_{\mathrm{SC}} ;
$$

note that $\mathcal{S}_{\text {symm }}^{\mathrm{NC}}(\Omega) \supseteq \mathcal{S}_{\text {symm }}(\Omega)$. We also define a non-conforming skeleton space $\mathcal{S}^{\mathrm{NC}} \supseteq$ $\mathcal{S}(\Omega)$ as

$$
\begin{aligned}
\mathcal{S}^{\mathrm{NC}}(\Omega) \equiv \mathcal{S}^{\mathrm{PR}}(\Omega) & \\
\oplus \operatorname{span}\left\{\psi_{i, j, k}, \quad\left(n_{\mathrm{A}, i, j}^{\gamma}+1\right) \leq k \leq \mathcal{N}_{i, j}^{\gamma}, 1 \leq j \leq n_{i}^{\gamma}, 1 \leq i \leq I\right\} & \\
& \equiv \operatorname{span}\left\{\Psi_{i}^{\prime}, \quad 1 \leq i \leq n_{\mathrm{NC}}\right\} .
\end{aligned}
$$

Hence for

$$
e_{0}^{\mathrm{NC}}(\mu) \equiv \arg \min _{\nu \in \mathcal{S}_{\text {symm }}^{\mathrm{NC}}(\Omega)} \mathcal{J}(v ; \mu)
$$


(recall the "broken" definition of $a(\cdot, \cdot ; \mu)$ in $(8))$ we must have

$$
\mathcal{J}\left(e_{0}^{\mathrm{NC}}(\mu) ; \mu\right) \leq \mathcal{J}\left(e_{0}^{h}(\mu) ; \mu\right)
$$

and thus $a\left(e_{0}^{\mathrm{NC}}(\mu), e_{0}^{\mathrm{NC}}(\mu) ; \mu\right) \geq a\left(e_{0}^{h}(\mu), e_{0}^{h}(\mu) ; \mu\right)$. This first relaxation of (78) not only provides a bound on the energy of the error field, but also accommodates efficient bound calculation thanks to the non-conforming space $\mathcal{S}_{\text {symm }}^{\mathrm{NC}}(\Omega)$.

A second relaxation step is required to obtain a computationally tractable error bound. To this end we introduce a bound conditioner, the bilinear form $b_{\mu}: \mathcal{S}_{\text {symm }}^{\mathrm{NC}}(\Omega) \times \mathcal{S}_{\text {symm }}^{\mathrm{NC}} \rightarrow$ $\mathbb{R}$, defined as

$$
b_{\mu}(\cdot, \cdot) \equiv a\left(\cdot, \cdot ; \mu_{\text {ref }}^{\mu}\right)
$$

for a reference parameter value $\mu_{\text {ref }}^{\mu} \in \mathcal{D}$. Note that here, $b_{\mu}(\cdot, \cdot)$ depends implicitly on $\mu$ through the parameter-dependent reference parameter $\mu_{\text {ref }}^{\mu}$. In fact, an important innovation of this paper is this multi-reference parameter bound conditioner: in the online stage, we optimally select $\mu_{\text {ref }}^{\mu}$ from a database of a few candidate reference parameters (through a discrete enumeration procedure); we discuss the selection of $\mu_{\text {ref }}^{\mu}$ further in the "Computational procedures" subsection below. We also define

$$
\lambda_{\min }(\mu) \equiv \min _{\nu \in \mathcal{S}_{\text {symm }}^{\mathrm{NC}}(\Omega)} \frac{a(v, v ; \mu)}{b_{\mu}(\nu, v)} .
$$

We then introduce a modified functional

$$
\mathcal{J}_{b}(v ; \mu) \equiv \frac{\lambda_{\min }(\mu)}{2} b_{\mu}(v, v)-\left(f(v)-a\left(u^{\mathrm{PR}}(\mu), v ; \mu\right)\right), \quad \forall v \in \mathcal{S}_{\mathrm{symm}}^{\mathrm{NC}}(\Omega),
$$

and we consider the minimization

$$
\bar{e}_{0}^{\mathrm{NC}}(\mu) \equiv \arg \min _{v \in \mathcal{S}_{\text {symm }}^{\mathrm{NC}}(\Omega)} \mathcal{J}_{b}(v ; \mu) .
$$

By the definition of $\lambda_{\min }(\mu)$ in (86) it is clear that $\mathcal{J}_{b}(v ; \mu) \leq \mathcal{J}(v ; \mu)$ for all $v \in$ $\mathcal{S}_{\text {symm }}^{\mathrm{NC}}(\Omega)$. Thus in particular, since $\bar{e}_{0}^{\mathrm{NC}}(\mu)$ is the minimizer,

$$
\mathcal{J}_{b}\left(\bar{e}_{0}^{\mathrm{NC}}(\mu) ; \mu\right) \leq \mathcal{J}_{b}\left(e_{0}^{\mathrm{NC}}(\mu) ; \mu\right) \leq \mathcal{J}\left(e_{0}^{\mathrm{NC}}(\mu) ; \mu\right) \leq \mathcal{J}\left(e_{0}(\mu) ; \mu\right),
$$

where the last inequality follows from (84). Consequently, we obtain the energy-norm error bound

$$
\lambda_{\min }(\mu) b_{\mu}\left(\bar{e}_{0}^{\mathrm{NC}}(\mu), \bar{e}_{0}^{\mathrm{NC}}(\mu)\right) \geq a\left(e_{0}^{h}(\mu), e_{0}^{h}(\mu) ; \mu\right)
$$

where the field variable $\bar{e}_{0}^{\mathrm{NC}}(\mu) \in \mathcal{S}_{\mathrm{symm}}^{\mathrm{NC}}(\Omega)$ - a presumably rather good approximation to the original error field $e_{0}^{h}(\mu)[12]-$ satisfies the elliptic problem $b_{\mu}\left(\bar{e}_{0}^{\mathrm{NC}}(\mu), v\right)=$ $\lambda_{\min }(\mu)^{-1}\left(f(v ; \mu)-a\left(u^{\mathrm{PR}}(\mu), v ; \mu\right)\right)$ for all $v \in \mathcal{S}_{\text {symm }}^{\mathrm{NC}}(\Omega)$. Equivalently, because of the Galerkin orthogonality in (19),

$$
b_{\mu}\left(\bar{e}_{0}^{\mathrm{NC}}(\mu), v\right)=\frac{1}{\lambda_{\min }(\mu)}\left(f(v ; \mu)-a\left(u^{\mathrm{PR}}(\mu), v ; \mu\right)\right), \quad \forall v \in \mathcal{S}^{\mathrm{NC}}(\Omega) .
$$

Thanks to incorporation of the modes related to rigid-body motion in our port space bases (presuming $n_{\mathrm{A}, p}^{\Gamma} \geq 6$ on all global ports $\Gamma_{p}, 1 \leq p \leq n^{\Gamma}$ ) we expect in general (and for a particular system, we computationally verify) that (91) is well-posed; for the simpler class of problems with scalar-valued fields we demonstrate this well-posedness in [12]. The RB-error-free bound given in (90) (together with (91)) is the basis on which we in the next subsection extend our error estimation framework to the general case of non-zero $\mathrm{RB}$ errors and furthermore to certain outputs of interest. 
In order to implement this error bound, and to facilitate incorporation of RB-induced error contributions, we now interpret the error bound (90) in terms of algebraic quantities. To this end, we first note that, for any $v(\mu)=\sum_{p=1}^{n^{\Gamma}} \sum_{k=1}^{\mathcal{N}_{p}^{\Gamma}} \mathbb{V}_{p, k}(\mu) \Phi_{p, k}(\mu)-$ that is, for any $v(\mu) \in \mathcal{S}_{\text {symm }}(\Omega)$ with coefficients $\mathbb{V}(\mu)$ - we have

$$
a(\nu(\mu), v(\mu) ; \mu)=\mathbb{V}(\mu)^{\mathrm{T}} \mathbb{A}(\mu) \mathbb{V}(\mu) ;
$$

we refer to the right-hand side of (92) as the "Schur energy" of $\mathbb{V}(\mu)$. It shall prove convenient to introduce the zero-extended solution vectors

$$
\hat{\mathbb{U}}_{0}^{\mathrm{PR}}(\mu) \equiv\left[\begin{array}{c}
\mathbb{U}^{\mathrm{PR}}(\mu) \\
\mathbf{0}
\end{array}\right] \in \mathbb{R}^{n_{\mathrm{SC}}}, \quad \text { and } \quad \hat{\mathbb{U}}_{0}^{\mathrm{PR}, \mathrm{NC}}(\mu) \equiv\left[\begin{array}{c}
\mathbb{U}^{\mathrm{PR}}(\mu) \\
\mathbf{0}
\end{array}\right] \in \mathbb{R}^{n_{\mathrm{NC}}},
$$

in which all but the first $n_{\mathrm{A}}$ entries are explicitly set to zero. We also define the error coefficient vector

$$
\mathbb{E}_{0}(\mu) \equiv \mathbb{U}(\mu)-\hat{\mathbb{U}}_{0}^{\mathrm{PR}}(\mu) \in \mathbb{R}^{n_{\mathrm{SC}}}
$$

such that the error (75) can be written $e_{0}^{h}(\mu)=\sum_{p=1}^{n^{\Gamma}} \sum_{k=1}^{\mathcal{N}_{p}^{\Gamma}} \mathbb{E}_{0 ; p, k}(\mu) \Phi_{p, k}(\mu)$. Note here, we tacitly interpret (without loss of generality) $\mathbb{U}(\mu)$ such that the first $n_{\mathrm{A}}$ entries correspond to the $n_{\mathrm{A}}$ active degrees of freedom. The algebraic version of the error residual equation (77) is

$$
\mathbb{A}(\mu) \mathbb{E}_{0}(\mu)=\mathbb{R}_{0}(\mu),
$$

where the residual vector is given as

$$
\mathbb{R}_{0}(\mu)=\mathbb{F}(\mu)-\mathbb{A}(\mu) \hat{\mathbb{U}}_{0}^{\mathrm{PR}}(\mu) ;
$$

note that, thanks to (92) and the fact that $e_{0}^{h}(\mu) \in \mathcal{S}_{\text {symm }}(\Omega)$, (95) is equivalent to (77).

We now introduce a non-conforming matrix $\mathbb{A}^{\mathrm{NC}}(\mu) \in \mathbb{R}^{n_{\mathrm{NC}} \times n_{\mathrm{NC}}}$ and vector $\mathbb{F}^{\mathrm{NC}}(\mu) \in$ $\mathbb{R}^{n_{\mathrm{NC}}}$ as

$$
\begin{aligned}
& \mathbb{A}_{i, j}^{\mathrm{NC}}(\mu)=a\left(\Phi_{j}^{\prime}(\mu), \Phi_{i}^{\prime}(\mu) ; \mu\right), \\
& \mathbb{F}_{i}^{\mathrm{NC}}(\mu)=f\left(\Phi_{i}^{\prime}(\mu) ; \mu\right)-\sum_{l=1}^{I} a\left(b_{l}^{f ; h}\left(\mu_{l}\right), \Phi_{i}^{\prime}(\mu) ; \mu\right),
\end{aligned}
$$

for $1 \leq i, j \leq n_{\mathrm{NC}}$. Note that $a\left(\Phi_{j}^{\prime}(\mu), \Phi_{i}^{\prime}(\mu) ; \mu\right)=a\left(\Phi_{j}^{\prime}(\mu), \Psi_{i}^{\prime} ; \mu\right)$ because of the Galerkin orthogonality in (19), and thus $\mathbb{A}^{\mathrm{NC}}(\mu)$ is indeed the non-conforming version of the Schur complement matrix $\mathbb{A}(\mu)$ in $(26)$; similarly, note that $f\left(\Phi_{i}^{\prime}(\mu) ; \mu\right)-$ $\sum_{l=1}^{I} a\left(b_{l}^{f ; h}\left(\mu_{l}\right), \Phi_{i}^{\prime}(\mu) ; \mu\right)=f\left(\Psi_{i}^{\prime} ; \mu\right)-\sum_{l=1}^{I} a\left(b_{l}^{f ; h}\left(\mu_{l}\right), \Psi_{i}^{\prime}(\mu) ; \mu\right)$ because of $(18)$ and the fact that $\Phi_{i}^{\prime}(\mu)-\Psi_{i}^{\prime}$ vanish on ports, and thus $\mathbb{F}^{\mathrm{NC}}(\mu)$ is the non-conforming version of the vector $\mathbb{F}(\mu)$ in $(26)$.

We further define a non-conforming reference matrix

$$
\mathbb{B}_{\mu}^{\mathrm{NC}} \equiv \mathbb{A}^{\mathrm{NC}}\left(\mu_{\mathrm{ref}}^{\mu}\right),
$$

which corresponds to the bilinear form $b_{\mu}(\cdot, \cdot)$. We also introduce a non-conforming residual vector $\mathbb{R}_{0}^{\mathrm{NC}}(\mu) \in \mathbb{R}^{n_{\mathrm{NC}}}$ as

$$
\mathbb{R}_{0 ; i}^{\mathrm{NC}}(\mu)=f\left(\Phi_{i}^{\prime}(\mu)\right)-a\left(u^{\mathrm{PR}}(\mu), \Phi_{i}^{\prime}(\mu) ; \mu\right), \quad 1 \leq i \leq n_{\mathrm{NC}}
$$

note that $\mathbb{R}_{0}^{\mathrm{NC}}(\mu)=\mathbb{F}^{\mathrm{NC}}(\mu)-\mathbb{A}^{\mathrm{NC}}(\mu) \hat{\mathbb{U}}_{0}^{\mathrm{PR}}(\mu)$. 
Next, we introduce a (unknown) coefficient vector $\overline{\mathbb{E}}_{0}^{\mathrm{NC}}(\mu) \in \mathbb{R}^{n_{\mathrm{NC}}}$ such that

$$
\bar{e}_{0}^{\mathrm{NC}}(\mu)=\sum_{i=1}^{n_{\mathrm{NC}}} \overline{\mathbb{E}}_{0 ; i}(\mu) \Phi_{i}^{\prime}(\mu)
$$

Thus from (91), (99), and (100) we obtain

$$
\overline{\mathbb{E}}_{0}^{\mathrm{NC}}(\mu)=\frac{1}{\lambda_{\min }(\mu)}\left(\mathbb{B}_{\mu}^{\mathrm{NC}}\right)^{-1} \mathbb{R}_{0}^{\mathrm{NC}}(\mu) .
$$

Similarly to (92), we note that for any $v(\mu)=\sum_{i=1}^{n_{\mathrm{NC}}} \mathbb{V}_{i}(\mu) \Phi_{i}^{\prime}(\mu)-$ that is, for any $\nu(\mu) \in \mathcal{S}_{\text {symm }}^{\mathrm{NC}}(\Omega)-$ we have

$$
a(v(\mu), v(\mu) ; \mu)=\mathbb{V}(\mu)^{\mathrm{T}} \mathbb{A}^{\mathrm{NC}}(\mu) \mathbb{V}(\mu) .
$$

Hence in particular, since $\bar{e}_{0}^{\mathrm{NC}}(\mu) \in \mathcal{S}_{\text {symm }}^{\mathrm{NC}}(\Omega)$, we obtain

$$
\begin{aligned}
\lambda_{\min }(\mu) b_{\mu}\left(\bar{e}_{0}^{\mathrm{NC}}(\mu), \bar{e}_{0}^{\mathrm{NC}}(\mu)\right) & =\lambda_{\min }(\mu) \overline{\mathbb{E}}_{0}^{\mathrm{NC}}(\mu)^{\mathrm{T}} \mathbb{B}_{\mu}^{\mathrm{NC}} \overline{\mathbb{E}}_{0}^{\mathrm{NC}}(\mu) \\
& =\frac{1}{\lambda_{\min }(\mu)} \mathbb{R}_{0}^{\mathrm{NC}}(\mu)^{\mathrm{T}}\left(\mathbb{B}_{\mu}^{\mathrm{NC}}\right)^{-1} \mathbb{R}_{0}^{\mathrm{NC}}(\mu) .
\end{aligned}
$$

Further, since $e_{0}^{h}(\mu) \in \mathcal{S}_{\text {symm }}$, we may invoke (92) and write

$$
a\left(e_{0}(\mu), e_{0}(\mu) ; \mu\right)=\mathbb{E}_{0}(\mu)^{\mathrm{T}} \mathbb{A}(\mu) \mathbb{E}_{0}(\mu) .
$$

Finally, we note that $\lambda_{\min }(\mu)$ of $(86)$ is the smallest eigenvalue associated with the generalized eigenproblem

$$
\mathbb{A}^{\mathrm{NC}}(\mu) \mathbb{V}(\mu)=\lambda(\mu) \mathbb{B}_{\mu}^{\mathrm{NC}} \text {. }
$$

The algebraic interpretation of the port reduction error bound (90) is thus

$$
\frac{1}{\lambda_{\min }(\mu)} \mathbb{R}_{0}^{\mathrm{NC}}(\mu)^{\mathrm{T}}\left(\mathbb{B}_{\mu}^{\mathrm{NC}}\right)^{-1} \mathbb{R}_{0}^{\mathrm{NC}}(\mu) \geq \mathbb{E}_{0}(\mu)^{\mathrm{T}} \mathbb{A}(\mu) \mathbb{E}_{0}(\mu)
$$

We note that the bound (107) necessitates a solve $\left(\mathbb{B}_{\mu}^{N C}\right)^{-1} \mathbb{R}_{0}^{N C}(\mu)$ of dimension $n_{\mathrm{NC}} \geq$ $n_{\mathrm{SC}}$. However, this solve may be performed efficiently thanks to $i$ ) the non-conforming skeleton space $\mathcal{S}^{\mathrm{NC}}(\Omega)$ which in a natural way allows component-local elimination of all degrees of freedom that do not couple at shared global ports; and ii) the quasi parameterindependent bound conditioner matrix $\mathbb{B}_{\mu}^{N C}$ associated with the bilinear form $b_{\mu}$, which allows offline pre-factorization for all these component-local solves. And furthermore, in actual practice we invoke not $\lambda_{\min }(\mu)$ but rather a computationally tractable eigenvalue lower bound $\tilde{\lambda}_{\min , \mathrm{LB}}(\mu) \leq \lambda_{\min }(\mu)$. We consider computational aspects of our error estimation framework in more detail in the "Computational procedures" subsection below.

\section{RB error contribution - A Posteriori error estimators}

We now modify (107) in order to obtain an efficiently computable a posteriori error bound which is also valid in the presence of RB error contributions. First, as we in the SCRBE context only have access to an approximation of the FE Schur complement system, the residual can not be computed exactly and we thus instead compute a residual approximation together with bounds on associated RB-error-induced residual perturbation terms. Second, we introduce a lower bound (valid under an eigenvalue proximity assumption) for the eigenvalue $\lambda_{\min }(\mu)$ which is based on the solution to a port-reduced eigenproblem, an approximate eigenproblem residual, and bounds on associated RB-error-induced eigenproblem residual perturbation terms. 
Moreover, in the presence of RB error contributions the error in the Schur energy is not equal to the energy of the error in the field, and thus in addition to a bound on the former we require a bound on additional RB perturbation terms to obtain a bound for the latter. Further, we develop in this section, from our Schur energy error bound, a new bound on port-restricted compliance outputs. For this output bound we must take into account that we in this paper (in contrast to in [12]) employ $\mathcal{S}^{\mathrm{PR}}(\Omega)$ rather than $\tilde{\mathcal{S}}_{\text {symm }}^{\text {PR }}(\Omega) \subset \tilde{\mathcal{S}}_{\text {symm }}(\Omega)$ (the former being a port-reduced version of the latter, which is defined in (51)) as our skeleton space. Finally, we introduce asymptotically rigorous error estimators, by which we reduce computational cost by neglecting typically very small quadratic RB error bound contributions.

To begin, we define the error field as

$$
e^{h}(\mu) \equiv u^{h}(\mu)-\tilde{u}^{\mathrm{PR}}(\mu) .
$$

It is again convenient to introduce the zero-extended solution vectors,

$$
\hat{\mathbb{U}}^{\mathrm{PR}}(\mu) \equiv\left[\begin{array}{c}
\tilde{\mathbb{U}}^{\mathrm{PR}}(\mu) \\
\mathbf{0}
\end{array}\right] \in \mathbb{R}^{n_{\mathrm{SC}}}, \quad \text { and } \quad \hat{\mathbb{U}}^{\mathrm{PR}, \mathrm{NC}}(\mu) \equiv\left[\begin{array}{c}
\tilde{\mathbb{U}}^{\mathrm{PR}}(\mu) \\
\mathbf{0}
\end{array}\right] \in \mathbb{R}^{n_{\mathrm{NC}}},
$$

in which the solution $\tilde{\mathbb{U}}^{\mathrm{PR}}(\mu)$ of (64) is extended by $n_{\mathrm{SC}}-n_{\mathrm{A}}$ and $n_{\mathrm{NC}}-n_{\mathrm{A}}$ zeros, respectively. We may then write

$$
e^{h}(\mu)=\sum_{i=1}^{I}\left(b_{i}^{h_{i} f}\left(\mu_{i}\right)-\tilde{b}_{i}^{h: f}\left(\mu_{i}\right)\right)+\sum_{p=1}^{n^{\Gamma}} \sum_{k=1}^{\mathcal{N}_{p}^{\Gamma}}\left(\mathbb{U}_{p, k}(\mu) \Phi_{p, k}(\mu)-\hat{\mathbb{U}}_{p, k}^{\mathrm{PR}}(\mu) \tilde{\Phi}_{p, k}(\mu)\right),
$$

and we note that $e^{h}(\mu)$ is not a member of $\mathcal{S}_{\text {symm }}(\Omega)$ because of the errors in the RB bubble approximations. We also define a vector of error coefficients as

$$
\mathbb{E}(\mu) \equiv \mathbb{U}(\mu)-\hat{\mathbb{U}}^{\mathrm{PR}}(\mu) .
$$

We first develop a bound for the error in the Schur energy norm, $\sqrt{\mathbb{E}(\mu)^{\mathrm{T}} \mathbb{A}(\mu) \mathbb{E}(\mu)}$, through perturbations of the left-hand side of (107). We subsequently modify this bound to obtain a bound on $\left\|e^{h}(\mu)\right\|_{\mu}$; note the former is not equivalent to the latter because $e^{h}(\mu)$ is not a member of $\mathcal{S}_{\text {symm }}(\Omega)$.

The usual error-residual relationship still holds in the presence of RB error contributions. In this case the relevant error-residual equation is

$$
\mathbb{A}(\mu) \mathbb{E}(\mu)=\mathbb{R}(\mu),
$$

where the residual vector is given as

$$
\mathbb{R}(\mu)=\mathbb{F}(\mu)-\mathbb{A}(\mu) \hat{\mathbb{U}}^{\mathrm{PR}}(\mu) .
$$

The difference between (95) and (112) is rather subtle: the former features the residual associated with the RB-error-free solution vector $\hat{\mathbb{U}}_{0}^{\mathrm{PR}}(\mu)$ (never computationally realized), while the latter features the residual associated with the RB-error-affected SCRBE solution vector $\hat{\mathbb{U}}^{\mathrm{PR}}(\mu)$ (computed in practice). The non-conforming version of the residual is

$$
\mathbb{R}^{\mathrm{NC}}(\mu) \equiv \mathbb{F}^{\mathrm{NC}}(\mu)-\mathbb{A}^{\mathrm{NC}}(\mu) \hat{\mathbb{U}}^{\mathrm{PR}}(\mu) .
$$

Next, we redefine our quasi parameter-independent (due to online reference parameter selection) bound conditioner matrix $\mathbb{B}_{\mu}^{\mathrm{NC}}$ from the previous subsection as $\mathbb{B}_{\mu}^{\mathrm{NC}}=$ 
$\tilde{\mathbb{A}}^{\mathrm{NC}}\left(\mu_{\text {ref }}^{\mu}\right)$; note that any SPD matrix may serve as our bound conditioner, and thus the RB approximations now present in $\mathbb{B}_{\mu}^{N C}$ do not necessitate modifications to the error bound expression (and therefore the $\mathbb{B}_{\mu}^{N C}$ of the previous subsection did not bear a subscript ${ }_{0}$ ). Henceforth, the eigenproblem (106) is interpreted with this redefined $\mathbb{B}_{\mu}^{\mathrm{NC}}$ as the righthand side matrix, and $\lambda_{\min }(\mu)$ is interpreted as the associated smallest eigenvalue. In the presence of RB error contributions, (107) now becomes

$$
\frac{\mathbb{R}^{\mathrm{NC}}(\mu)^{\mathrm{T}}\left(\mathbb{B}_{\mu}^{\mathrm{NC}}\right)^{-1} \mathbb{R}^{\mathrm{NC}}(\mu)}{\lambda_{\min }(\mu)} \geq \mathbb{E}(\mu)^{\mathrm{T}} \mathbb{A}(\mu) \mathbb{E}(\mu) .
$$

To bound the error in the Schur energy, we must thus, based on residual and eigenvalue approximations, develop upper and lower bounds for the numerator and denominator, respectively, of the left-hand side of (115).

We first consider the approximation to the non-conforming residual $\mathbb{R}^{\mathrm{NC}}(\mu)$. As we do not have access to $\mathbb{F}^{\mathrm{NC}}(\mu)$ and $\mathbb{A}^{\mathrm{NC}}(\mu)$ as defined in (97) and (98), but rather to RBapproximated versions $\tilde{\mathbb{F}}^{\mathrm{NC}}(\mu) \approx \mathbb{F}^{\mathrm{NC}}(\mu)$ and $\tilde{\mathbb{A}}^{\mathrm{NC}}(\mu) \approx \mathbb{A}^{\mathrm{NC}}(\mu)$, we introduce our approximation based on $\tilde{\mathbb{F}}^{\mathrm{NC}}(\mu) \approx \mathbb{F}^{\mathrm{NC}}(\mu)$ and $\tilde{\mathbb{A}}^{\mathrm{NC}}(\mu) \approx \mathbb{A}^{\mathrm{NC}}(\mu)$ as

$$
\tilde{\mathbb{R}}^{\mathrm{NC}}(\mu)=\tilde{\mathbb{F}}^{\mathrm{NC}}(\mu)-\tilde{\mathbb{A}}^{\mathrm{NC}}(\mu) \hat{\mathbb{U}}^{\mathrm{PR}, \mathrm{NC}}(\mu)
$$

such that $\tilde{\mathbb{R}}^{\mathrm{NC}}(\mu)=\mathbb{R}^{\mathrm{NC}}(\mu)+\delta \mathbb{R}^{\mathrm{NC}}(\mu)$. Here,

$$
\delta \mathbb{R}^{\mathrm{NC}}(\mu)=\tilde{\mathbb{F}}^{\mathrm{NC}}(\mu)-\mathbb{F}^{\mathrm{NC}}(\mu)+\left(\mathbb{A}^{\mathrm{NC}}(\mu)-\tilde{\mathbb{A}}^{\mathrm{NC}}(\mu)\right) \hat{\mathbb{U}}^{\mathrm{PR}, \mathrm{NC}}(\mu)
$$

is an RB-error-induced perturbation term. We may readily from standard RB error bounds [5,6] develop bounds on these perturbation quantities; we introduce a vector $\sigma(\mu)$ such that, for any $\mu \in \mathcal{D}$,

$$
\sigma_{i}(\mu) \geq\left|\delta \mathbb{R}_{i}^{N C}(\mu)\right|, \quad 1 \leq i \leq n_{\mathrm{NC}} .
$$

We next consider the approximation to the eigenvalue $\lambda_{\min }(\mu)$. Again, as we do not in practice have access to $\mathbb{A}^{\mathrm{NC}}(\mu)$, and furthermore as we wish to avoid solution of a full eigenproblem of dimension $n_{\mathrm{NC}}$, we consider an approximation $\tilde{\lambda}_{\min }^{\mathrm{PR}}(\mu)$ to $\lambda_{\min }(\mu)$ given as the smallest eigenvalue associated with the port-reduced SCRBE eigenproblem

$$
\tilde{\mathbb{A}}^{\mathrm{PR}}(\mu) \mathbb{V}(\mu)=\tilde{\lambda}^{\mathrm{PR}}(\mu) \mathbb{B}_{\mu}^{\mathrm{PR}} \mathbb{V}(\mu) ;
$$

here, $\mathbb{B}_{\mu}^{\mathrm{PR}}$ denotes the block of $\mathbb{B}_{\mu}^{\mathrm{NC}}$ associated with "Active" degrees of freedom. We denote by $\mathbb{V}_{\text {min }}^{\mathrm{PR}}(\mu)$ the eigenvector associated with $\tilde{\lambda}_{\min }^{\mathrm{PR}}(\mu)$, and we assume the normalization $\mathbb{V}_{\text {min }}^{\mathrm{PR}}(\mu)^{\mathrm{T}} \mathbb{B}_{\mu}^{\mathrm{PR}} \mathbb{V}_{\text {min }}^{\mathrm{PR}}(\mu)=1$. We also introduce an approximate eigenproblem residual

$$
\tilde{\mathbb{R}}_{\text {eig }}^{\mathrm{NC}}(\mu)=\tilde{\mathbb{A}}^{\mathrm{NC}}(\mu) \hat{\mathbb{V}}_{\min }^{\mathrm{PR}}(\mu)-\tilde{\lambda}_{\min }^{\mathrm{PR}}(\mu) \mathbb{B}_{\mu}^{\mathrm{NC}} \hat{\mathbb{V}}_{\min }^{\mathrm{PR}}(\mu),
$$

in which $\hat{\mathbb{V}}_{\text {min }}^{\mathrm{PR}}(\mu) \in \mathbb{R}^{n_{\mathrm{NC}}}$ is a zero-expanded version of $\mathbb{V}_{\min }^{\mathrm{PR}}(\mu) \in \mathbb{R}^{n_{\mathrm{A}}}$. Note that the exact eigenproblem residual is given as $\mathbb{R}_{\text {eig }}^{\mathrm{NC}}(\mu)=\mathbb{A}^{\mathrm{NC}} \hat{\mathbb{V}}_{\text {min }}^{\mathrm{PR}}(\mu)-\tilde{\lambda}_{\min }^{\mathrm{PR}}(\mu) \mathbb{B}_{\mu}^{\mathrm{NC}} \hat{\mathbb{V}}_{\text {min }}^{\mathrm{PR}}(\mu)$, and we may thus define a vector of $\mathrm{RB}$ perturbation terms $\delta \mathbb{R}_{\text {eig }}^{\mathrm{NC}}(\mu)$ such that $\mathbb{R}_{\text {eig }}^{N C}(\mu)=\tilde{\mathbb{R}}_{\text {eig }}^{N C}(\mu)+\delta \mathbb{R}_{\text {eig }}^{N C}(\mu)$. We may then develop bounds on these RB-errorinduced perturbation quantities - we introduce a vector $\sigma_{\text {eig }}(\mu)$ such that, for any $\mu \in \mathcal{D}$,

$$
\sigma_{\mathrm{eig}, i}(\mu) \geq\left|\delta \mathbb{R}_{\mathrm{eig}, i}^{\mathrm{NC}}(\mu)\right|, \quad 1 \leq i \leq n_{\mathrm{NC}} .
$$

We now obtain a computable eigenvalue lower bound in 
Lemma 1. Let $C>0$ be such that

$$
\delta \mathbb{R}_{\mathrm{eig}}^{\mathrm{NC}}(\mu)^{\mathrm{T}}\left(\mathbb{B}_{\mu}^{\mathrm{NC}}\right)^{-1} \delta \mathbb{R}_{\mathrm{eig}}^{\mathrm{NC}}(\mu) \leq C\left\|\delta \mathbb{R}_{\mathrm{eig}}^{\mathrm{NC}}(\mu)\right\|_{2}^{2},
$$

assume that

$$
\left|\lambda_{\min }^{\mathrm{PR}}(\mu)-\lambda_{\min }(\mu)\right| \leq\left|\lambda_{\min }^{\mathrm{PR}}(\mu)-\lambda(\mu)\right|,
$$

for all $\lambda(\mu)$ which satisfy (106) (with the redefined $\mathbb{B}_{\mu}^{\mathrm{NC}}$ ), and let

$$
\begin{aligned}
& \lambda_{\min , \mathrm{LB}}(\mu ; C) \equiv \tilde{\lambda}_{\min }^{\mathrm{PR}}(\mu) \\
& -\sqrt{\tilde{\mathbb{R}}_{\text {eig }}^{\mathrm{NC}}(\mu)^{\mathrm{T}}\left(\mathbb{B}_{\mu}^{N C}\right)^{-1} \tilde{\mathbb{R}}_{\text {eig }}^{\mathrm{NC}}(\mu)+2 \sigma_{\text {eig }}(\mu)^{\mathrm{T}}\left|\left(\mathbb{B}_{\mu}^{N C}\right)^{-1} \tilde{\mathbb{R}}_{\text {eig }}^{N C}(\mu)\right|+C\left\|\sigma_{\text {eig }}(\mu)\right\|_{2}^{2}} .
\end{aligned}
$$

Then

$$
\lambda_{\min , \mathrm{LB}}(\mu ; C) \leq \lambda_{\min }(\mu) .
$$

Proof. We refer to ([12], Proposition 1) for the proof, and we note that a similar residualbased eigenvalue bound has been developed in [18] for the standard eigenproblem.

With the residual approximation $\tilde{\mathbb{R}}(\mu)$, associated RB error bounds $\sigma(\mu)$, and the eigenvalue lower bound $\lambda_{\min , \mathrm{LB}}(\mu ; C)$ above, we may now obtain a computable bound for the left-hand side of (115) and thus the error in the Schur energy norm in

Proposition 1. Let $C>0$ be a computable constant such that

$$
\begin{aligned}
& \delta \mathbb{R}^{\mathrm{NC}}(\mu)^{\mathrm{T}}\left(\mathbb{B}_{\mu}^{\mathrm{NC}}\right)^{-1} \delta \mathbb{R}^{\mathrm{NC}}(\mu) \leq C\left\|\delta \mathbb{R}^{\mathrm{NC}}(\mu)\right\|_{2}^{2}, \\
& \delta \mathbb{R}_{\text {eig }}^{\mathrm{NC}}(\mu)^{\mathrm{T}}\left(\mathbb{B}_{\mu}^{\mathrm{NC}}\right)^{-1} \delta \mathbb{R}_{\text {eig }}^{\mathrm{NC}}(\mu) \leq C\left\|\delta \mathbb{R}_{\text {eig }}^{\mathrm{NC}}(\mu)\right\|_{2}^{2} .
\end{aligned}
$$

Then define

$$
\Delta^{\mathbb{U}}(\mu ; C) \equiv \sqrt{\frac{\tilde{\mathbb{R}}^{N C}(\mu)^{\mathrm{T}}\left(\mathbb{B}_{\mu}^{N C}\right)^{-1} \tilde{\mathbb{R}}^{N C}(\mu)+2 \sigma(\mu)^{\mathrm{T}}\left|\left(\mathbb{B}_{\mu}^{N C}\right)^{-1} \tilde{\mathbb{R}}^{N C}(\mu)\right|+C\|\boldsymbol{\sigma}(\mu)\|_{2}^{2}}{\lambda_{\min , \mathrm{LB}}(\mu ; C)}} .
$$

Then if the assumption (123) holds, we have

$$
\sqrt{\mathbb{E}(\mu)^{\mathrm{T}} \mathbb{A}(\mu) \mathbb{E}(\mu)} \leq \Delta^{\mathbb{U}}(\mu ; C) .
$$

Proof. We merely note here that the numerator in (128) is an upper bound for the numerator in (115), and that $\lambda_{\min , \mathrm{LB}}(\mu ; C) \leq \lambda_{\min }(\mu)$ is a lower bound for the denominator in (115). We refer to ([12], Appendix A) for the detailed proof.

We proceed to bound the energy of the error in the field. Since $e^{h}(\mu)$ is not a member of $\mathcal{S}_{\text {symm }}(\Omega)$, a small modification to (128) is necessary to obtain a bound for $\left\|e^{h}(\mu)\right\|_{\mu}$. To this end, we introduce additional RB perturbation terms

$$
\begin{aligned}
\Delta b^{f}(\mu) & \equiv \sum_{i=1}^{I}\left(b_{i}^{f ; h}(\mu)-\tilde{b}_{i}^{f}(\mu)\right) \\
\Delta \Phi_{\mathrm{A}}(\mu) & \equiv \sum_{p=1}^{n^{\Gamma}} \sum_{k=1}^{n_{\mathrm{A}, p}^{\Gamma}} \tilde{\mathbb{U}}_{\mathrm{A}, p, k}(\mu)\left(\Phi_{p, k}(\mu)-\tilde{\Phi}_{p, k}(\mu)\right) ;
\end{aligned}
$$


we also introduce an RB error bound [6] $\kappa(\mu)$ such that, for any $\mu \in \mathcal{D}$,

$$
\kappa(\mu) \geq\left\|\Delta b^{f}(\mu)+\Delta \Phi_{\mathrm{A}}(\mu)\right\|_{\mu} .
$$

We then introduce our bound for the energy of the error field in

Proposition 2. Define $\Delta^{u}(\mu ; C)$ as

$$
\Delta^{u}(\mu ; C) \equiv \sqrt{\left(\Delta^{\mathbb{U}}(\mu ; C)\right)^{2}+\kappa(\mu)^{2}} .
$$

where $\kappa(\mu)$ is given in (132). Then if the assumption (123) holds, we have

$$
\left\|e^{h}(\mu)\right\|_{\mu} \leq \Delta^{u}(\mu ; C) .
$$

Proof. We refer to ([12], Appendix A) for the proof.

Next, we develop a bound for the error in port-restricted compliance outputs. To this end we introduce a matrix $\sigma_{\mathbb{A}}(\mu) \in \mathbb{R}^{n_{\mathrm{A}} \times n_{\mathrm{A}}}$ such that

$$
\sigma_{\mathbb{A}, i, j}(\mu) \geq\left|\mathbb{A}_{i, j}(\mu)-\tilde{\mathbb{A}}_{i, j}(\mu)\right|, \quad 1 \leq i, j \leq n_{\mathrm{A}} .
$$

We then state

\section{Proposition 3. Let}

$$
\Delta^{s}(\mu ; C) \equiv\left(\Delta^{\mathbb{U}}(\mu ; C)\right)^{2}+\left|\tilde{\mathbb{U}}^{\mathrm{PR}}(\mu)\right|^{\mathrm{T}} \boldsymbol{\sigma}_{\mathbb{A}}(\mu)\left|\tilde{\mathbb{U}}^{\mathrm{PR}}(\mu)\right|
$$

(in which $|\cdot|$ denotes entry-wise absolute value and not vector modulus). Assume that the source $f(\cdot ; \mu)$ is restricted to ports such that $b_{i}^{f ; h}\left(\mu_{i}\right)=0,1 \leq i \leq I$. The error in a port-restricted compliance output $\tilde{s}^{\mathrm{PR}}(\mu)=f\left(\tilde{u}^{\mathrm{PR}}(\mu) ; \mu\right)$ can then be bounded as

$$
\left|s^{h}(\mu)-\tilde{s}^{\mathrm{PR}}(\mu)\right| \leq \Delta^{s}(\mu ; C)
$$

Proof. We provide here a full proof as in the present paper (skeleton space $\mathcal{S}^{\mathrm{PR}}(\Omega)$ ) the proof is different from a related proof in [12] (skeleton space $\tilde{\mathcal{S}}_{\text {symm }}(\Omega)$ ).

We first note that

$$
e^{h}(\mu)=\Delta b^{f}(\mu)+\Delta \Phi_{\mathrm{A}}(\mu)+\sum_{p=1}^{n^{\Gamma}} \sum_{k=1}^{\mathcal{N}_{p}^{\Gamma}} \mathbb{E}_{p, k}(\mu) \Phi_{p, k}(\mu)
$$

note in the port-restricted output case considered here, $\Delta b^{f}(\mu)=0$. For the compliance output error, we may then write (using symmetry of $a(\cdot, \cdot ; \mu)$ )

$$
\begin{aligned}
s^{h}(\mu)- & \tilde{s}^{\mathrm{PR}}(\mu)=a\left(u^{h}(\mu), e^{h}(\mu) ; \mu\right) \\
& =a\left(e^{h}(\mu), u^{h}(\mu) ; \mu\right)=a\left(e^{h}(\mu), e^{h}(\mu) ; \mu\right)+a\left(e^{h}(\mu), \tilde{u}^{\mathrm{PR}}(\mu) ; \mu\right),
\end{aligned}
$$

and thus by (138) (and again symmetry of $a(\cdot, \cdot ; \mu)$ )

$$
\begin{aligned}
s^{h}(\mu)-\tilde{s}^{\mathrm{PR}}(\mu) & =\mathbb{E}(\mu)^{\mathrm{T}} \mathbb{A}(\mu) \mathbb{E}(\mu)+a\left(\Delta \Phi_{\mathrm{A}}(\mu), \Delta \Phi_{\mathrm{A}}(\mu) ; \mu\right) \\
& +2 \sum_{p=1}^{n^{\Gamma}} \sum_{k=1}^{\mathcal{N}_{p}^{\Gamma}} a\left(\Phi_{p, k}(\mu), \Delta \Phi_{\mathrm{A}}(\mu) ; \mu\right)+a\left(e^{h}(\mu), \tilde{u}^{\mathrm{PR}}(\mu) ; \mu\right) .
\end{aligned}
$$

We note that $e^{h}(\mu)$ is not Galerkin-orthogonal to $\tilde{u}^{\mathrm{PR}}(\mu)$ because $\tilde{u}^{\mathrm{PR}}(\mu)$ (even in the case $\left.b_{i}^{f ; h}\left(\mu_{i}\right)=0\right)$ is not a member of the skeleton test space $\mathcal{S}^{\mathrm{PR}}(\Omega)$. We thus do not 
obtain equality between the compliance output error and the squared energy of the error field in (139). This is the key difference between the compliance output error bound result here and in [12]; in [12], we invoke the skeleton space $\tilde{\mathcal{S}}_{\text {symm }}^{\mathrm{PR}}(\Omega) \subset \tilde{\mathcal{S}}^{\mathrm{PR}}(\Omega)$ (the latter is defined in (51)) of which $\tilde{u}^{\mathrm{PR}}(\mu)$ is a member (for port-restricted compliance such that $\left.\tilde{b}_{i}^{f}(\mu)=0\right)$, and thus we directly obtain this equality.

We next note that $\left.\Delta \Phi_{\mathrm{A}}(\mu)\right|_{\Omega_{i}}$ vanish on all ports and thus is a member of the FE bubble space associated with instantiated component $i$. From the Galerkin orthogonality (19) we then conclude that the third term on the right-hand side of (140) is equal to zero, and we obtain

$s^{h}(\mu)-\tilde{s}^{\mathrm{PR}}(\mu)=\mathbb{E}(\mu)^{\mathrm{T}} \mathbb{A}(\mu) \mathbb{E}(\mu)+a\left(\Delta \Phi_{\mathrm{A}}(\mu), \Delta \Phi_{\mathrm{A}}(\mu) ; \mu\right)+a\left(e^{h}(\mu), \tilde{u}^{\mathrm{PR}}(\mu) ; \mu\right)$

We now consider the two right-most terms on the right-hand side of (141) (we omit the $\mu$-dependence for simplicity of exposition). We first obtain

$$
\begin{aligned}
& a\left(\Delta \Phi_{\mathrm{A}}, \Delta \Phi_{\mathrm{A}}\right)+a\left(e^{h}, \tilde{u}^{\mathrm{PR}}\right) \\
& =\sum_{p=1}^{n^{\Gamma}} \sum_{k=1}^{n_{\mathrm{A}, p}^{\Gamma}} \sum_{p^{\prime}=1}^{n^{\Gamma}} \sum_{k^{\prime}=1}^{n_{\mathrm{A}, p}^{\Gamma}} \tilde{\mathbb{U}}_{p, k}^{\mathrm{PR}} \tilde{\mathbb{U}}_{p^{\prime}, k^{\prime}}^{\mathrm{PR}} a\left(\Phi_{p, k}-\tilde{\Phi}_{p, k}, \Phi_{p^{\prime}, k^{\prime}}-\tilde{\Phi}_{p^{\prime}, k^{\prime}}\right) \\
& \quad+\sum_{p=1}^{n^{\Gamma}} \sum_{k=1}^{n_{\mathrm{A}, p}^{\Gamma}} \sum_{p^{\prime}=1}^{n^{\Gamma}} \sum_{k^{\prime}=1}^{n_{\mathrm{A}, p}^{\Gamma}} \tilde{\mathbb{U}}_{p, k}^{\mathrm{PR}} \tilde{\mathbb{U}}_{p^{\prime}, k^{\prime}}^{\mathrm{PR}} a\left(\Phi_{p, k}-\tilde{\Phi}_{p, k}, \tilde{\Phi}_{p^{\prime}, k^{\prime}}\right) \\
& \quad+\sum_{p=1}^{n^{\Gamma}} \sum_{k=1}^{\mathcal{N}_{p}^{\Gamma}} \sum_{p^{\prime}=1}^{n^{\Gamma}} \sum_{k^{\prime}=1}^{n_{\mathrm{A}, p}^{\Gamma}} \mathbb{E}_{p, k} \tilde{\mathbb{U}}_{p^{\prime}, k^{\prime}}^{\mathrm{PR}} a\left(\Phi_{p, k}, \tilde{\Phi}_{p^{\prime}, k^{\prime}}\right),
\end{aligned}
$$

by the expression for $e^{h}(\mu)$ in (138) (for $\Delta b^{f}(\mu)=0$ ) and the definition of $\Delta \Phi_{\mathrm{A}}(\mu)$ in (131). For the first two terms on the right-hand side of (142) we obtain

$$
\begin{aligned}
& \sum_{p=1}^{n^{\Gamma}} \sum_{k=1}^{n_{\mathrm{A}, p}^{\Gamma}} \sum_{p^{\prime}=1}^{n^{\Gamma}} \sum_{k^{\prime}=1}^{n_{\mathrm{A}, p}^{\Gamma}} \tilde{\mathbb{U}}_{p, k}^{\mathrm{PR}} \tilde{\mathbb{U}}_{p^{\prime}, k^{\prime}}^{\mathrm{PR}} a\left(\Phi_{p, k}-\tilde{\Phi}_{p, k}, \Phi_{p^{\prime}, k^{\prime}}-\tilde{\Phi}_{p^{\prime}, k^{\prime}}\right) \\
& +\sum_{p=1}^{n^{\Gamma}} \sum_{k=1}^{n_{\mathrm{A}, p}^{\Gamma}} \sum_{p^{\prime}=1}^{n^{\Gamma}} \sum_{k^{\prime}=1}^{n_{\mathrm{A}, p}^{\Gamma}} \tilde{\mathbb{U}}_{p, k}^{\mathrm{PR}} \tilde{\mathbb{U}}_{p^{\prime}, k^{\prime}}^{\mathrm{PR}} a\left(\Phi_{p, k}-\tilde{\Phi}_{p, k}, \tilde{\Phi}_{p^{\prime}, k^{\prime}}\right) \\
& =\sum_{p=1}^{n^{\Gamma}} \sum_{k=1}^{n_{\mathrm{A}, p}^{\Gamma}} \sum_{p^{\prime}=1}^{n^{\Gamma}} \sum_{k^{\prime}=1}^{n_{\mathrm{A}, p}^{\Gamma}} \tilde{\mathbb{U}}_{p, k}^{\mathrm{PR}} \tilde{\mathbb{U}}_{p^{\prime}, k^{\prime}}^{\mathrm{PR}} a\left(\Phi_{p, k}-\tilde{\Phi}_{p, k}, \Phi_{p^{\prime}, k^{\prime}}\right) \\
& =\sum_{p=1}^{n^{\Gamma}} \sum_{k=1}^{n_{\mathrm{A}, p}} \sum_{p^{\prime}=1}^{n^{\Gamma}} \sum_{k^{\prime}=1}^{n_{\mathrm{A}, p}^{\Gamma}} \tilde{\mathbb{U}}_{p, k}^{\mathrm{PR}} \tilde{\mathbb{U}}_{p^{\prime}, k^{\prime}}^{\mathrm{PR}} a\left(\Phi_{p^{\prime}, k^{\prime}}, \Phi_{p, k}-\tilde{\Phi}_{p, k}\right)=0,
\end{aligned}
$$

where in the second step we invoke symmetry of $a(\cdot, \cdot ; \mu)$ and in the final step the Galerkin orthogonality (19). For the last term on the right-hand side of (142) we obain

$$
\begin{aligned}
\sum_{p=1}^{n^{\Gamma}} \sum_{k=1}^{\mathcal{N}_{p}^{\Gamma}} \sum_{p^{\prime}=1}^{n^{\Gamma}} \sum_{k^{\prime}=1}^{n_{\mathrm{A}, p}^{\Gamma}} \mathbb{E}_{p, k} \tilde{\mathbb{U}}_{p^{\prime}, k^{\prime}}^{\mathrm{PR}} a\left(\Phi_{p, k}, \tilde{\Phi}_{p^{\prime}, k^{\prime}}\right) \\
=\sum_{p=1}^{n^{\Gamma}} \sum_{k=1}^{\mathcal{N}_{p}^{\Gamma}} \sum_{p^{\prime}=1}^{n^{\Gamma}} \sum_{k^{\prime}=1}^{n_{\mathrm{A}, p}^{\Gamma}} \mathbb{E}_{p, k} \tilde{\mathbb{U}}_{p^{\prime}, k^{\prime}}^{\mathrm{PR}} a\left(\Phi_{p, k}, \Phi_{p^{\prime}, k^{\prime}}\right)=\mathbb{E}^{\mathrm{T}} \mathbb{A} \hat{\mathbb{U}}^{\mathrm{PR}},
\end{aligned}
$$


where we again exploit Galerkin orthogonality with respect to $\Phi_{p^{\prime}, k^{\prime}}(\mu)-\tilde{\Phi}_{p^{\prime}, k^{\prime}}(\mu)$.

We note that $\mathbb{A}(\mu) \mathbb{E}(\mu)=\mathbb{A}(\mu)\left(\mathbb{U}(\mu)-\hat{\mathbb{U}}^{\mathrm{PR}}(\mu)\right)=\mathbb{F}(\mu)-\mathbb{A}(\mu) \hat{\mathbb{U}}^{\mathrm{PR}}(\mu)$. With (141), (142), (143), and (144) (and symmetry of $\mathbb{A}(\mu)$ ) we then obtain

$$
\begin{aligned}
s^{h}(\mu)-\tilde{s}^{\mathrm{PR}}(\mu) & =\mathbb{E}(\mu)^{\mathrm{T}} \mathbb{A}(\mu) \mathbb{E}(\mu)+\mathbb{E}(\mu)^{\mathrm{T}} \mathbb{A}(\mu) \hat{\mathbb{U}}^{\mathrm{PR}}(\mu) \\
& =\mathbb{E}(\mu)^{\mathrm{T}} \mathbb{A}(\mu) \mathbb{E}(\mu)+\left(\mathbb{F}(\mu)-\mathbb{A}(\mu) \hat{\mathbb{U}}^{\mathrm{PR}}(\mu)\right)^{\mathrm{T}} \hat{\mathbb{U}}^{\mathrm{PR}}(\mu) .
\end{aligned}
$$

In the case of a port-restricted compliance, we have $b_{i}^{f ; h}\left(\mu_{i}\right)=\tilde{b}_{i}^{f}\left(\mu_{i}\right)=0$ and thus also $\mathbb{F}(\mu)=\tilde{\mathbb{F}}(\mu)$. It is furthermore straightforward to show that $(\tilde{\mathbb{F}}(\mu)-$ $\left.\tilde{\mathbb{A}}(\mu) \hat{\mathbb{U}}^{\mathrm{PR}}(\mu)\right)^{\mathrm{T}} \hat{\mathbb{U}}^{\mathrm{PR}}(\mu)=0$ because the port-reduced SCRBE solution vector $\tilde{\mathbb{U}}^{\mathrm{PR}}(\mu)$ (that is, the non-zero coefficients of $\hat{\mathbb{U}}^{\mathrm{PR}}(\mu)$ ) satisfies (64) exactly. We thus obtain in this case

$$
\begin{aligned}
\left(\mathbb{F}(\mu)-\mathbb{A}(\mu) \hat{\mathbb{U}}^{\mathrm{PR}}(\mu)\right)^{\mathrm{T}} \hat{\mathbb{U}}^{\mathrm{PR}}(\mu)= & \left(\tilde{\mathbb{F}}(\mu)-\mathbb{A}(\mu) \hat{\mathbb{U}}^{\mathrm{PR}}(\mu)\right)^{\mathrm{T}} \hat{\mathbb{U}}^{\mathrm{PR}}(\mu) \\
= & \left(\tilde{\mathbb{F}}(\mu)-\tilde{\mathbb{A}}(\mu) \hat{\mathbb{U}}^{\mathrm{PR}}(\mu)+\tilde{\mathbb{A}}(\mu) \hat{\mathbb{U}}^{\mathrm{PR}}(\mu)\right. \\
& \left.-\mathbb{A}(\mu) \hat{\mathbb{U}}^{\mathrm{PR}}(\mu)\right)^{\mathrm{T}} \hat{\mathbb{U}}^{\mathrm{PR}}(\mu) \\
= & \left((\tilde{\mathbb{A}}(\mu)-\mathbb{A}(\mu)) \hat{\mathbb{U}}^{\mathrm{PR}}(\mu)\right)^{\mathrm{T}} \hat{\mathbb{U}}^{\mathrm{PR}}(\mu) .
\end{aligned}
$$

From (145) and (146) (and symmetry of $\tilde{\mathbb{A}}(\mu)$ and $\mathbb{A}(\mu)$ ) we then conclude that

$$
s^{h}(\mu)-\tilde{s}^{\mathrm{PR}}(\mu)=\mathbb{E}(\mu)^{\mathrm{T}} \mathbb{A}(\mu) \mathbb{E}(\mu)+\hat{\mathbb{U}}^{\mathrm{PR}}(\mu)^{\mathrm{T}}(\tilde{\mathbb{A}}(\mu)-\mathbb{A}(\mu)) \hat{\mathbb{U}}^{\mathrm{PR}}(\mu),
$$

which, with the triangle inequality and (135), yields the desired result.

We do not in the present paper consider bounds on more general outputs.

We reiterate that Lemma 1, Proposition 1, Proposition 2, and Proposition 3 all provide rigorous bounds under the eigenvalue proximity assumption given in (123). These bounds necessitate computation of a bound $C$ for Rayleigh quotients associated with $\left(\mathbb{B}_{\mu}^{N C}\right)^{-1}$, for which we may choose $C=1 / \lambda_{\min }\left(\mathbb{B}_{\mu}^{N C}\right)$, where $\lambda_{\min }\left(\mathbb{B}_{\mu}^{N C}\right)$ is the smallest eigenvalue associated with $\mathbb{B}_{\mu}^{\mathrm{NC}}$. Unfortunately, this choice for $C$ is typically a rather pessimistic Rayleigh quotient bound, ${ }^{\mathrm{h}}$ and furthermore calculation of $\lambda_{\min }\left(\mathbb{B}_{\mu}^{\mathrm{NC}}\right)$ requires considerable (albeit, as discussed in the next section, not onerous) computational cost. However, we note that the terms which multiply $C$ in (124) and (128), as well as the term $\kappa(\mu)^{2}$ in (133), are quadratic in RB error bound contributions and thus presumably small compared to the terms that are linear in RB error bound contributions.

We thus introduce asymptotically rigorous error estimators, in which we choose to neglect these terms: we set $C=0$ in (124) and (128) to obtain a Schur energy error estimator $\Delta^{\mathbb{U}}(\mu ; 0)$; we then obtain an estimator for the energy of the error field as

$$
\Delta^{u ; 0}(\mu) \equiv \Delta^{\mathbb{U}}(\mu ; 0)
$$

in which we also neglect the term $\kappa(\mu)^{2}$ in (133); and finally we obtain an estimator for the port-restricted compliance output error as

$$
\Delta^{s ; 0}(\mu) \equiv \Delta^{s}(\mu ; 0) .
$$

In actual practice, RB errors are typically rather small, and we shall thus for our largescale numerical results in this paper employ the error estimators (148) and (149). 


\section{Computational procedures}

The main computational costs associated with our a posteriori error estimation framework derive from the two non-conforming solves $\left(\mathbb{B}_{\mu}^{\mathrm{NC}}\right)^{-1} \tilde{\mathbb{R}}_{\mathrm{eig}}^{\mathrm{NC}}(\mu)$ and $\left(\mathbb{B}_{\mu}^{\mathrm{NC}}\right)^{-1} \tilde{\mathbb{R}}^{\mathrm{NC}}(\mu)$ required in (124) and in the numerator of (128), respectively, and from the calculation of the smallest eigenvalue $\tilde{\lambda}_{\min }^{\mathrm{PR}}(\mu)$ of (119). We now discuss the former in more detail; for the latter we employ an implementation of a Krylov-Schur (inverted spectrum) iterative solver from the SLEPc library [19].

For our discussion here it is convenient to first introduce a particular interpretation of the non-port-reduced SCRBE system matrix and right-hand side as

$$
\tilde{\mathbb{A}}(\mu)=\left[\begin{array}{ll}
\tilde{\mathbb{A}}_{\mathrm{A}, \mathrm{A}}(\mu) & \tilde{\mathbb{A}}_{\mathrm{A}, \mathrm{I}}(\mu) \\
\tilde{\mathbb{A}}_{\mathrm{I}, \mathrm{A}}(\mu) & \tilde{\mathbb{A}}_{\mathrm{I}, \mathrm{I}}(\mu)
\end{array}\right], \quad \tilde{\mathbb{F}}(\mu)=\left[\begin{array}{cc}
\tilde{\mathbb{F}}_{\mathrm{A}}(\mu) \\
\tilde{\mathbb{F}}_{\mathrm{I}}(\mu)
\end{array}\right] .
$$

Here, the matrix block $\tilde{\mathbb{A}}_{\mathrm{A}, \mathrm{A}}(\mu)=\tilde{\mathbb{A}}^{\mathrm{PR}}(\mu)$ is the "Active" matrix block which we invoke for our port-reduced SCRBE approximation, the blocks $\tilde{\mathbb{A}}_{\mathrm{I}, \mathrm{A}}(\mu)$ and $\tilde{\mathbb{A}}_{\mathrm{I}, \mathrm{A}}(\mu)$ correspond to couplings between the "Active" and "Inactive" degrees of freedom, and the block $\tilde{\mathbb{A}}_{\mathrm{I}, \mathrm{I}}(\mu)$ is associated only with "Inactive" degrees of freedom. Note that the interpretation (150) simply corresponds to a particular ordering of (54).

In the particular case of only two instantiated components, the system matrix $\tilde{\mathbb{A}}(\mu)$ may be written as

$$
\tilde{\mathbb{A}}(\mu)=\left[\begin{array}{ll}
\tilde{\mathbb{A}}_{\mathrm{A}, \mathrm{A}}^{1}\left(\mu_{1}\right)+\mathbb{A}_{\mathrm{A}, \mathrm{A}}^{2}\left(\mu_{2}\right) & \tilde{\mathbb{A}}_{\mathrm{A}, \mathrm{I}}^{1}\left(\mu_{1}\right)+\tilde{\mathbb{A}}_{\mathrm{A}, \mathrm{I}}^{2}\left(\mu_{2}\right) \\
\tilde{\mathbb{A}}_{\mathrm{I}, \mathrm{A}}^{1}\left(\mu_{1}\right)+\mathbb{A}_{\mathrm{I}, \mathrm{A}}^{2}\left(\mu_{2}\right) & \tilde{\mathbb{A}}_{\mathrm{I}, \mathrm{I}}^{1}\left(\mu_{1}\right)+\tilde{\mathbb{A}}_{\mathrm{I}, \mathrm{I}}^{2}\left(\mu_{2}\right)
\end{array}\right],
$$

where each submatrix $\tilde{\mathbb{A}}_{*, *^{\prime}}^{i}(\mu)$ is a matrix block associated with instantiated component $i, i=1,2$. The non-conforming matrix $\tilde{\mathbb{A}}^{\mathrm{NC}}(\mu)$ for this two-component system is then

$$
\tilde{\mathbb{A}}^{N C}(\mu)=\left[\begin{array}{ccc}
\tilde{\mathbb{A}}_{\mathrm{A}, \mathrm{A}}^{1}\left(\mu_{1}\right)+\tilde{\mathbb{A}}_{\mathrm{A}, \mathrm{A}}^{2}\left(\mu_{2}\right) & \tilde{\mathbb{A}}_{\mathrm{A}, \mathrm{I}}^{1}\left(\mu_{1}\right) & \tilde{\mathbb{A}}_{\mathrm{A}, \mathrm{I}}^{2}\left(\mu_{2}\right) \\
\tilde{\mathbb{A}}_{\mathrm{I}, \mathrm{A}}^{1}\left(\mu_{1}\right) & \tilde{\mathbb{A}}_{\mathrm{I}, \mathrm{I}}^{1}\left(\mu_{1}\right) & \mathbf{0} \\
\tilde{\mathbb{A}}_{\mathrm{I}, \mathrm{A}}^{2}\left(\mu_{2}\right) & \mathbf{0} & \tilde{\mathbb{A}}_{\mathrm{I}, \mathrm{I}}^{2}\left(\mu_{2}\right)
\end{array}\right] .
$$

Note that the difference between (151) and (152) is that the latter does not couple "Inactive" port degrees of freedom.

For the computation of the residual approximation $\tilde{\mathbb{R}}^{\mathrm{NC}}(\mu)$ in (116) we note that

$$
\begin{aligned}
& \tilde{\mathbb{R}}^{N C}(\mu)=\left[\begin{array}{c}
\tilde{\mathbb{R}}_{A}(\mu) \\
\tilde{\mathbb{R}}_{I}^{1}(\mu) \\
\tilde{\mathbb{R}}_{I}^{2}(\mu)
\end{array}\right] \\
& =\left[\begin{array}{l}
\tilde{\mathbb{F}}_{\mathrm{A}}(\mu) \\
\tilde{\mathbb{F}}_{\mathrm{I}}^{1}\left(\mu_{1}\right) \\
\tilde{\mathbb{F}}_{\mathrm{I}}^{2}\left(\mu_{2}\right)
\end{array}\right]-\left[\begin{array}{ccc}
\tilde{\mathbb{A}}_{\mathrm{A}, \mathrm{A}}(\mu) & \tilde{\mathbb{A}}_{\mathrm{A}, \mathrm{I}}^{1}\left(\mu_{1}\right) & \tilde{\mathbb{A}}_{\mathrm{A}, \mathrm{I}}^{2}\left(\mu_{2}\right) \\
\tilde{\mathbb{A}}_{\mathrm{I}, \mathrm{A}}^{1}\left(\mu_{1}\right) & \tilde{\mathbb{A}}_{\mathrm{I}, \mathrm{I}}^{1}\left(\mu_{1}\right) & \mathbf{0} \\
\tilde{\mathbb{A}}_{\mathrm{I}, \mathrm{A}}^{2}\left(\mu_{2}\right) & \mathbf{0} & \tilde{\mathbb{A}}_{\mathrm{I}, \mathrm{I}}^{2}\left(\mu_{2}\right)
\end{array}\right]\left[\begin{array}{l}
\tilde{\mathbb{U}}^{\mathrm{PR}}(\mu) \\
\mathbf{0} \\
\mathbf{0}
\end{array}\right] \\
& =\left[\begin{array}{c}
\tilde{\mathbb{F}}_{\mathrm{A}}(\mu)-\tilde{\mathbb{A}}_{\mathrm{A}, \mathrm{A}}(\mu) \tilde{\mathbb{U}}^{\mathrm{PR}}(\mu) \\
\tilde{\mathbb{F}}_{\mathrm{I}}\left(\mu_{1}\right)-\tilde{\mathbb{A}}_{\mathrm{I}, \mathrm{A}}^{1}\left(\mu_{1}\right) \tilde{\mathbb{U}}^{\mathrm{PR}}(\mu) \\
\tilde{\mathbb{F}}_{\mathrm{I}}^{2}\left(\mu_{2}\right)-\tilde{\mathbb{A}}_{\mathrm{I}, \mathrm{A}}^{2}\left(\mu_{2}\right) \tilde{\mathbb{U}}^{\mathrm{PR}}(\mu)
\end{array}\right]=\left[\begin{array}{c}
\mathbf{0} \\
\tilde{\mathbb{F}}_{\mathrm{I}}^{1}\left(\mu_{1}\right)-\tilde{\mathbb{A}}_{\mathrm{I}, \mathrm{A}}^{(}\left(\mu_{1}\right) \tilde{\mathbb{U}}^{\mathrm{PR}, 1}(\mu) \\
\tilde{\mathbb{F}}_{\mathrm{I}}^{2}\left(\mu_{2}\right)-\tilde{\mathbb{A}}_{\mathrm{I}, \mathrm{A}}^{2}\left(\mu_{2}\right) \tilde{\mathbb{U}}^{\mathrm{PR}, 2}(\mu)
\end{array}\right],
\end{aligned}
$$

where $\tilde{\mathbb{U}}^{\mathrm{PR}, i}(\mu)$ is extracted from $\tilde{\mathbb{U}}^{\mathrm{PR}}(\mu)$ for the degrees of freedom associated with component $i$. Note that the first $n_{\mathrm{A}}$ entries in the residual vector are zero, and that we may obtain the local residuals $\tilde{\mathbb{R}}_{\mathrm{I}}^{i}(\mu)$ by component-local evaluation. The eigenproblem residual approximation $\tilde{\mathbb{R}}_{\text {eig }}^{N C}(\mu)$ admits a similar procedure. 
We now consider the system $\mathbb{B}_{\mu}^{N C} z(\mu)=\tilde{\mathbb{R}}^{\mathrm{NC}}(\mu)$, which we may write as

$$
\left[\begin{array}{ccc}
\mathbb{B}_{\mathrm{A}, \mathrm{A}}^{1}+\mathbb{B}_{\mathrm{A}, \mathrm{A}}^{2} & \mathbb{B}_{\mathrm{A}, \mathrm{I}}^{1} & \mathbb{B}_{\mathrm{A}, \mathrm{I}}^{2} \\
\mathbb{B}_{\mathrm{I}, \mathrm{A}}^{1} & \mathbb{B}_{\mathrm{I}, \mathrm{I}}^{1} & \mathbf{0} \\
\mathbb{B}_{\mathrm{I}, \mathrm{A}}^{2} & \mathbf{0} & \mathbb{B}_{\mathrm{I}, \mathrm{I}}^{2}
\end{array}\right]\left[\begin{array}{c}
z_{\mathrm{A}}(\mu) \\
z_{\mathrm{I}}^{1}(\mu) \\
z_{\mathrm{I}}^{2}(\mu)
\end{array}\right]=\left[\begin{array}{c}
\tilde{\mathbb{R}}_{\mathrm{A}}(\mu) \\
\tilde{\mathbb{R}}_{\mathrm{I}}^{1}(\mu) \\
\tilde{\mathbb{R}}_{\mathrm{I}}^{2}(\mu)
\end{array}\right],
$$

and we note that

$$
\begin{aligned}
{\left[\mathbb{B}_{\mathrm{A}, \mathrm{A}}^{1}+\mathbb{B}_{\mathrm{A}, \mathrm{A}}^{2}-\mathbb{B}_{\mathrm{A}, \mathrm{I}}^{1}\left(\mathbb{B}_{\mathrm{I}, \mathrm{I}}^{1}\right)^{-1} \mathbb{B}_{\mathrm{I}, \mathrm{A}}^{1}-\mathbb{B}_{\mathrm{A}, \mathrm{I}}^{2}\left(\mathbb{B}_{\mathrm{I}, \mathrm{I}}^{2}\right)^{-1} \mathbb{B}_{\mathrm{I}, \mathrm{A}}^{2}\right] z_{\mathrm{A}}(\mu) } \\
\quad=\tilde{\mathbb{R}}_{\mathrm{A}}(\mu)-\mathbb{B}_{\mathrm{A}, \mathrm{I}}^{1}\left(\mathbb{B}_{\mathrm{I}, \mathrm{I}}^{1}\right)^{-1} \tilde{\mathbb{R}}_{\mathrm{I}}^{1}(\mu)-\mathbb{B}_{\mathrm{A}, \mathrm{I}}^{2}\left(\mathbb{B}_{\mathrm{I}, \mathrm{I}}^{2}\right)^{-1} \tilde{\mathbb{R}}_{\mathrm{I}}^{2}(\mu) .
\end{aligned}
$$

We may thus obtain $z(\mu)$ by consideration of a second Schur complement: we first solve smaller local problems associated with each of the two components, and then a global problem of size $n_{\mathrm{A}}$ for $z_{\mathrm{A}}(\mu)$; we finally recover $z(\mu)$ by standard back-substitution as $z_{\mathrm{I}}^{i}(\mu)=\left(\mathbb{B}_{\mathrm{I}, \mathrm{I}}^{i}\right)^{-1}\left(\tilde{\mathbb{R}}_{\mathrm{I}}^{i}(\mu)-\mathbb{B}_{\mathrm{I}, \mathrm{A}}^{i} z_{\mathrm{A}}(\mu)\right)$. The extension of this procedure to a system with an arbitrary number of components and ports is straightforward.

An important innovation of this paper for our error bound framework is a multireference parameter bound conditioner. In fact, the system reference parameter value $\mu_{\text {ref }}^{\mu}$ shall be chosen online, based on a database of component-local reference parameter values $\hat{\mu}_{m, \text { ref }}^{t}, 1 \leq t \leq n_{m \text {,ref, }} 1 \leq m \leq M$. The component-local reference matrices $\mathbb{B}_{*, *}^{i}$ in (154) and (155) are thus chosen online from a database of $n_{m, \text { ref }}$ precomputed component-local matrices $\hat{\mathbb{B}}_{*, *}^{m, t}$ associated with the parameter values $\hat{\mu}_{m, \text { ref }}^{t} \in \hat{\mathcal{D}}_{m}$. For our numerical results of this paper, we choose the component reference parameters to minimize the Euclidean distance between $\mu_{\text {ref }}^{\mu}$ and $\mu$. This multi-reference parameter bound conditioner procedure significantly sharpens our error bound through a closer-to-unity smallest eigenvalue $\tilde{\lambda}_{\min }^{\mathrm{PR}}(\mu)$ (and associated eigenvalue bound) at only minor additional computational cost (note a related approach is considered in [14] in a different context).

The computational efficacy of our error bound framework is thus realized largely through the quasi parameter-independent and non-conforming operator $\mathbb{B}_{\mu}^{\mathrm{NC}}$. As for the SCRBE approximation framework, the computational procedures associated with the error bound framework naturally decouple into offline and online stages. We consider these stages as extensions of the offline and online approximation computational stages discussed earlier, and we now discuss each in more detail (we again refer to [12] for detailed online operation counts).

\section{Offline}

Off5. Online dataset preparation. For each archetype component we construct data to enable efficient assembly of the matrix blocks $\tilde{\mathbb{A}}_{\mathrm{I}, \mathrm{A}}^{i}(\mu)$ required for residual calculation in (153) (the matrix blocks $\tilde{\mathbb{A}}_{\mathrm{A}, \mathrm{A}}^{i}(\mu)$ are also required for residual calculation; however the associated data is already constructed in Off3). Note that the blocks $\tilde{\mathbb{A}}_{\mathrm{A}, \mathrm{I}}^{i}(\mu)$ and, more importantly, the blocks $\tilde{\mathbb{A}}_{\mathrm{I}, \mathrm{I}}^{i}(\mu)$, are not required for residual calculation. Hence the cost of this stage scales quadratically in $n_{\mathrm{A}}$ but only linearly in $n_{\mathrm{I}}$.

Off6. Bound conditioner preparation. For each archetype component $m, 1 \leq m \leq M$,

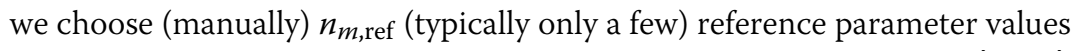
$\hat{\mu}_{m, \text { ref }}^{t}$ and compute associated bound conditioner reference matrices $\hat{\mathbb{B}}_{\mathrm{I}, \mathrm{I}}^{m, t}, \hat{\mathbb{B}}_{\mathrm{AA}}^{m, t}$, and $\hat{\mathbb{B}}_{\mathrm{A}, \mathrm{I}}^{m, t}, 1 \leq t \leq n_{m, \text { ref. }}$. 
We also perform and store the Cholesky factorization of each of the $\hat{\mathbb{B}}_{\mathrm{I}, \mathrm{I}}^{m, t}$, and we precompute the terms $\hat{\mathbb{B}}_{\mathrm{A}, \mathrm{I}}^{m, t}\left(\hat{\mathbb{B}}_{\mathrm{I}, \mathrm{I})}^{m, t}\right)^{-1} \hat{\mathbb{B}}_{\mathrm{I}, \mathrm{A}}^{m, t}$ required for assembly of the left-hand side of (155).

Off7. Data loading. We finally read the online datasets and error bound conditioner data (typically a few Gb combined) for all library components into computer memory to prepare for the online stage.

\section{Online}

On4. Port-reduced eigenproblem. We compute the smallest eigenvalue and associated eigenvector associated with (119) using a Krylov-Schur algorithm [19].

On5. Matrix and vector block assembly. Assemble component matrix and vector blocks $\tilde{\mathbb{A}}_{\mathrm{I}, \mathrm{A}}^{i}(\mu)$ and vectors $\tilde{\mathbb{F}}_{\mathrm{I}}^{i}(\mu)$ for each unique component instantiation; note that the "Active" component matrix and vector blocks $\tilde{\mathbb{A}}_{\mathrm{A}, \mathrm{A}}^{i}(\mu)$ are already assembled in On2. As in On2, we exploit "cloned" component instantiations to effectively reduce the number of component instantiations to $I_{\text {eff }} \ll I$.

On6. Residual calculation. Given the solution vector $\hat{\mathbb{U}}(\mu)$, the eigenvalue $\tilde{\lambda}_{\min }^{\mathrm{PR}}(\mu)$, and the associated (normalized and zero-expanded) eigenvector $\hat{\mathbb{V}}_{\min }^{\mathrm{PR}}(\mu)$, we calculate $\tilde{\mathbb{R}}^{\mathrm{NC}}(\mu)$ and $\tilde{\mathbb{R}}_{\text {eig }}^{\mathrm{NC}}(\mu)$ locally on each component.

On7. Non-conforming solves. We first choose the reference parameter value

$$
\mu_{\text {ref }}^{\mu}=\left(\hat{\mu}_{\mathcal{M}(1), \text { ref }}^{t_{1}}, \ldots, \hat{\mu}_{\mathcal{M}(I), \text { ref }}^{t_{I}}\right)
$$

(where $1 \leq t_{i} \leq n_{\mathcal{M}(i), \text { ref }}$ ) from the database of candidate component reference parameter values such that the Euclidean distance between each $\mu_{i}$ and $\hat{\mu}_{\mathcal{M}(i) \text {,ref }}^{t_{i}}$ is minimized. We then compute $\left(\mathbb{B}_{\mu}^{N C}\right)^{-1} \mathbb{R}^{N C}(\mu)$ and $\left(\mathbb{B}_{\mu}^{N C}\right)^{-1} \mathbb{R}_{\text {eig }}^{N C}(\mu)$ through component-local elimination of "Inactive" degrees of freedom as indicated in (155). Note that this step is particularly efficient thanks to the preparation in Off6.

On8. Calculation of $\lambda_{\min }\left(\mathbb{B}_{\mu}^{N C}\right)$. In the case that we wish to employ a rigorous error bound (we choose $C=1 / \lambda_{\min }\left(\mathbb{B}_{\mu}^{N C}\right.$ ) rather than $C=0$ ), we must also compute $\lambda_{\min }\left(\mathbb{B}_{\mu}^{N C}\right)$. Note that we may compute $\lambda_{\min }\left(\mathbb{B}_{\mu}^{N C}\right)$ rather efficiently through (typically) a few inverse power iterations, and hence only a few additional non-conforming solves. This procedure is applicable for $\lambda_{\min }\left(\mathbb{B}_{\mu}^{N C}\right)$ but not for $\lambda_{\min }\left(\tilde{\mathbb{A}}^{\mathrm{NC}}(\mu)\right)$ because the latter would have required expensive online formation of the $\tilde{\mathbb{A}}_{\mathrm{I}, \mathrm{I}}^{i}(\mu)$ component-local matrix blocks.

The computational cost associated with this online stage is typically dominated by On4 and On7. However for systems in which almost all components are unique - that is, $I_{\text {eff }}$ close to $I$ - the cost of matrix assembly in On5 is considerable. In any event, the error estimation online computational cost discussed here is typically larger than the approximation online computational cost discussed earlier (we report actual timings in the next section).

\section{Microtruss beam application}

We consider here application of our port-reduced SCRBE framework to structural analysis of a microtruss beam. The particular beam we consider is in practice manufactured from microcylinders that are welded together in a three-dimensional square array configuration to form a larger but light-weight truss structure; see Figure 3. Many examples 


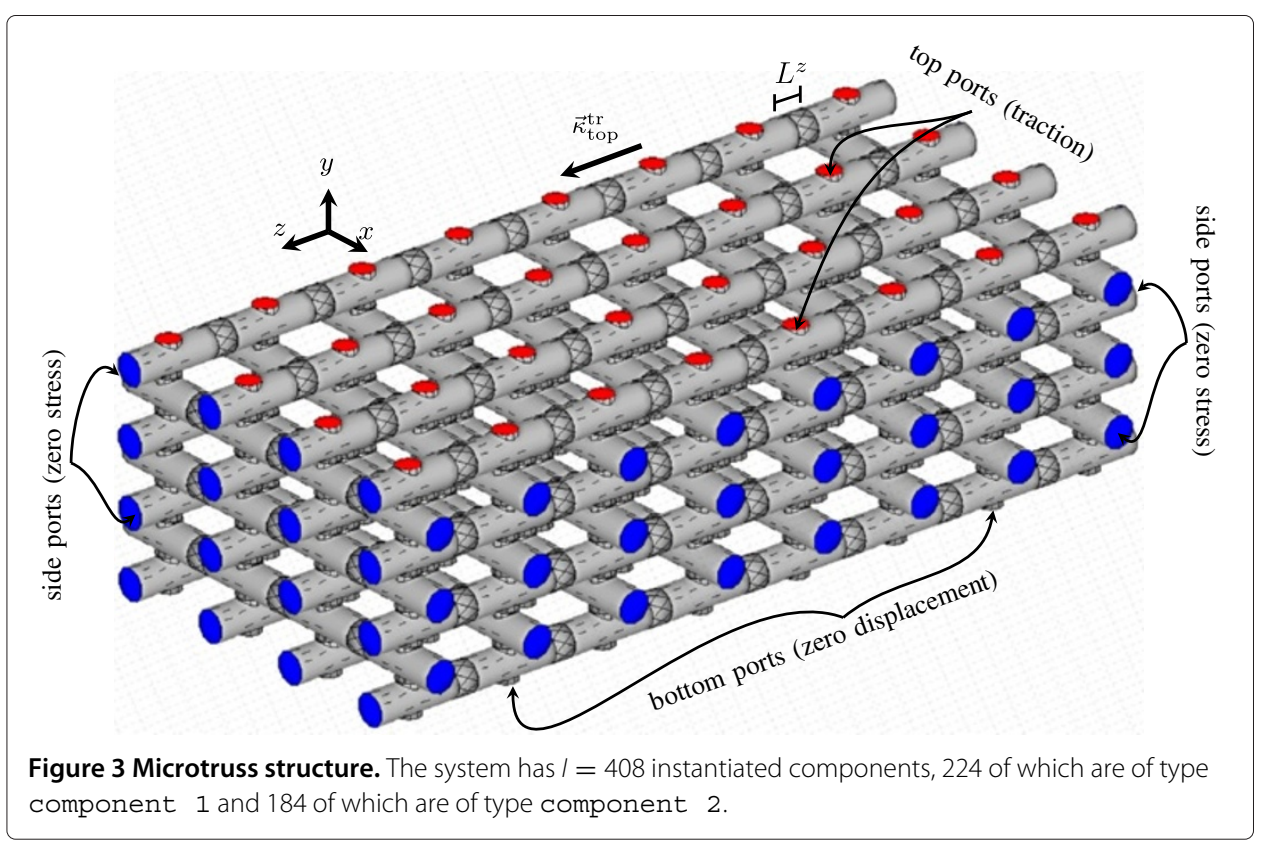

of microtruss structures exist in literature and in engineering, and our choice here is only one of numerous possibilities. We refer to [20-22] for analyses and manufacturing considerations for such structures, including the particular type we consider here.

The microtruss structure is a good fit for our methodology. First, the structure admits a very natural decomposition into components, and the macroscale beam is comprised of many identical or similar instantiations of the same component archetypes; thus typically we may obtain $I_{\text {eff }} \ll I$ which implies particularly effective treatment by the port-reduced SCRBE. Second, the behavior of the macroscale beam as a function of component parameters and system topology is non-trivial, and furthermore the solution may exhibit large localized stresses within the components; hence the fidelity of a full FE discretization provided by the port-reduced SCRBE framework at a fraction of the cost - is desired. Third, it is often of interest to assess performance in off-design conditions in particular in the presence of inevitable flaws, in which not just natural periodicity but departures from periodicity - well within the capabilities of the SCRBE - are important.

\section{Archetype component library}

Before we introduce our components, we consider the non-dimensionalization of the equations of isotropic linear elasticity for a "generic" archetype (and thus entities below bear ^s). To this end we first define the non-dimensional tensor $\hat{C}$ as

$$
\hat{C}_{i j k l} \equiv \frac{v}{(1+v)(1-2 v)} \delta_{i j} \delta_{k l}+\frac{1}{2(1+v)}\left(\delta_{i k} \delta_{j l}+\delta_{i l} \delta_{j k}\right), \quad 1 \leq i, j, k, l \leq 3,
$$

in which $v$ is the Poisson ratio (we choose $v=0.3$ for steel); the dimensional elasticity tensor is then given as the product $\hat{E}^{\operatorname{dim}} \hat{C}_{i j k l}$, where $\hat{E}^{\mathrm{dim}}$ is the Young's modulus. The associated stress tensor $\hat{\sigma}^{\operatorname{dim}}\left(\hat{u}^{\mathrm{dim}}\right)$, given the dimensional displacement $\hat{u}^{\mathrm{dim}}$, is defined as $\hat{\sigma}_{i j}^{\operatorname{dim}}\left(\hat{u}^{\operatorname{dim}}\right)=\hat{E}^{\operatorname{dim}} \hat{C}_{i j k l} \partial \hat{u}_{k}^{\operatorname{dim}} / \partial \hat{x}_{l}^{\operatorname{dim}}$.

We shall consider either homogeneous Dirichlet boundary conditions, or (port) tractions. In the latter case the boundary conditions are enforced through the stress tensor 
as $\hat{\sigma}_{i j}^{\operatorname{dim}} \hat{\mathbf{e}}_{j}^{\operatorname{dim}}=\hat{\boldsymbol{\kappa}}_{i}^{\mathrm{tr} ; \operatorname{dim}}\left(\hat{\mathbf{e}}_{j}^{\mathrm{dim}}\right.$ denotes the canonical vectors $)$ for a specified traction vector $\hat{\boldsymbol{\kappa}}^{\mathrm{tr} ; \operatorname{dim}}$.

To derive non-dimensional equations we introduce the dimensionless variables $\hat{x}=$ $\hat{x}^{\operatorname{dim}} / \hat{L}^{\operatorname{dim}, 0}, \hat{u}=\hat{u}^{\operatorname{dim}} / \hat{L}^{\operatorname{dim}, 0}, \hat{E}=\hat{E}^{\operatorname{dim}} / \hat{E}^{\operatorname{dim}, 0}, \hat{\sigma}=\hat{\sigma}^{\operatorname{dim}} / \hat{E}^{\operatorname{dim}, 0}$, and $\hat{\kappa}^{\operatorname{tr}}=\hat{\kappa}^{\operatorname{tr} ; \operatorname{dim}} / \hat{E}^{\operatorname{dim}, 0}$, where $\hat{L}^{\mathrm{dim}, 0}$ is a characteristic length, and $\hat{E}^{\mathrm{dim}, 0}$ is a characteristic Young's modulus. The non-dimensional traction boundary conditions then become $\sigma_{i j} \mathbf{n}_{j}=\boldsymbol{\kappa}_{i}^{\mathrm{tr}}$. Below, all our equations take a non-dimensional form.

We now introduce our (non-dimensional) archetype component library, which consists of the two three-dimensional isotropic linear-elastic components illustrated in Figure 4; note $L^{\text {dim, } 0}$ in Figure 4 is the characteristic length used in our non-dimensionalization.

The first archetype, component 1, is a "Steinmetz cylinder," and has four circular ports: the left and right ports are of type port 1 and the top and bottom ports are of type port 2 . For both port types, the reference port space dimension is $\mathcal{N}^{\beta}=219$ (73 mesh nodes). The FE discretization for component 1 has $\mathcal{N}_{1}=115,443$ degrees of freedom in linear hexahedral elements. Note in Figure 4 that the mesh is significantly refined where the weld stub meets the cylinder base in order to resolve potentially high stress concentrations in this area.

The archetype parameter vector for this component is

$$
\hat{\mu}_{1}=\left(\hat{E}_{1}, \hat{\kappa}_{1, \text { top }}^{\mathrm{tr}}\right) \text {, }
$$

where $\hat{E}_{1}=\hat{E}_{1}^{\operatorname{dim}} / \hat{E}_{1}^{\operatorname{dim}, 0}$ is a Young's modulus scaling parameter and $\hat{\boldsymbol{\kappa}}_{1 \text {,top }}^{\mathrm{tr}}$ is a directional traction applied on the top port. The archetype bilinear and linear forms associated with component 1 are, for all $\hat{w}, \hat{v} \in \hat{X}_{1}^{h}$, given as

$$
\begin{aligned}
\hat{a}_{1}\left(\hat{w}, \hat{v} ; \hat{\mu}_{1}\right) & =\hat{E}_{1} \int_{\hat{\Omega}_{1}} \frac{\partial \hat{w}^{i}}{\partial \hat{x}_{j}} \hat{C}_{i j k l} \frac{\partial \hat{v}^{k}}{\partial \hat{x}_{l}}, \\
\hat{f}_{1}\left(\hat{v} ; \hat{\mu}_{1}\right) & =\hat{\kappa}_{1, \mathrm{top}, i}^{\operatorname{tr}} \int_{\hat{\gamma}_{1, \mathrm{top}}} \hat{v}^{i},
\end{aligned}
$$

where $\hat{\gamma}_{1 \text {,top }}$ denotes the boundary associated with the top port. For the bound conditioner reference matrix blocks we consider a single reference parameter value $\hat{\mu}_{1 \text {,ref }}^{1}=$ $\hat{E}_{1, \text { ref }}^{1}=1\left(\right.$ thus $\left.n_{1, \text { ref }}=1\right)$.
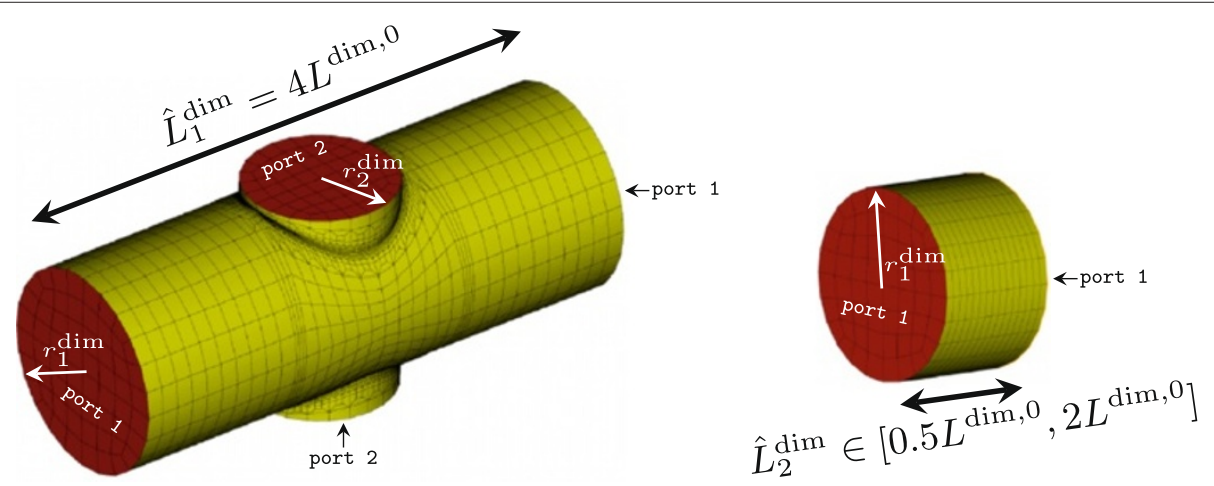

Figure 4 The (dimensional) archetype components for the microtruss library: component 1 (left) and component 2 (right). The port radius for port 1 and port 2 is $r_{1}^{\mathrm{dim}}=0.7405 \mathrm{~L}^{\mathrm{dim}}$ and $r_{2}^{d i m}=0.55 L^{d i m}$, respectively. 
We note that $\hat{Q}_{1}^{a}=1$; the Young's modulus parameter $\hat{E}_{1}$ is "free" in the sense that it enters outside the integral of (19). As a result, we may consider any value of $\hat{E}_{1}$ with only a single RB basis function in each of the RB approximation spaces. For $\hat{f}_{1}$ we note that $\hat{Q}_{1}^{f}=3$; however, as $\hat{f}_{1}$ is port-restricted, we obtain $\hat{b}_{1}^{f ; h}=0$.

We also note that $\hat{a}_{1}$ in (159) does not reflect the rigid-body mapping parameters required to "dock" instantiations of component 1 to the correct position in the system frame. To demonstrate this property more explicitly we consider the mapping $\mathcal{T}_{1}=$ $T_{1}^{\text {rot }} \mathcal{T}_{1}^{\text {def }}$, in which, for component $1, \mathcal{T}_{1}^{\text {def }}$ is pure translation; we introduce an associated rotation matrix $\mathbb{Q} \in \mathbb{R}^{3 \times 3}$ and a translation vector $\mathbb{T} \in \mathbb{R}^{3}$. For any coordinate $\hat{x} \in \hat{\Omega}_{1}$, we thus have $x_{i}=\mathbb{Q}_{i j}(\hat{x}+\mathbb{T})_{j}, 1 \leq i \leq 3$, where $x=\left(x_{1}, x_{3}, x_{3}\right) \in \Omega_{1}$ and $\Omega_{1}$ is the instantiated component domain; note that the Jacobian of the mapping, $\mathbb{Q}$, is unitary and thus $\operatorname{det} \mathbb{Q}=1$.

On $\Omega_{1}$, the instantiated component bilinear form reads, for any $w, v \in X_{1}$,

$$
a_{1}(w, v ; \mu)=E_{1} \int_{\Omega_{1}} \frac{\partial w^{i}}{\partial x_{j}} C_{i j k l} \frac{\partial v^{k}}{\partial x_{l}} \mathrm{~d} \Omega_{1}, \quad 1 \leq i, j, k, l \leq 3 .
$$

Here, $C$ is the elasticity tensor on the instantiated domain (i.e., in the system coordinates) such that [23]

$$
C_{i j k l}=\mathbb{Q}_{i i^{\prime}} \mathbb{Q}_{j j^{\prime}} \mathbb{Q}_{k k^{\prime}} \mathbb{Q}_{l l^{\prime}} \hat{C}_{i^{\prime} j^{\prime} k^{\prime} l^{\prime}}
$$

for $\hat{C}$ defined in (157). We recall from the Section "Component-based static condensation" that we apply $\mathcal{T}^{\text {rot }}$ to the dependent variables, and thus for any function $v \in X_{1}$ we write $v^{i}=\mathbb{Q}_{i j} \hat{v}^{j}$, where $\hat{v}=\left(\hat{v}^{1}, \hat{v}^{2}, \hat{v}^{3}\right) \in \hat{X}_{1}$. Starting from (161), we obtain in this case, for $w, v \in X_{1}$,

$$
\begin{aligned}
a_{1}\left(w, v ; \mu_{1}\right) & =E_{1} \int_{\hat{\Omega}}\left(\mathbb{Q}_{j j^{\prime}}^{-1} \frac{\partial w^{i}}{\partial \hat{x}_{j}}\right) C_{i j^{\prime} k l^{\prime}}\left(\mathbb{Q}_{l l^{\prime}}^{-1} \frac{\partial v^{k}}{\partial \hat{x}_{l}}\right)(\operatorname{det} \mathbb{Q}) \mathrm{d} \hat{\Omega} \\
& =E_{1} \int_{\hat{\Omega}}\left(\mathbb{Q}_{j j^{\prime}}^{-1} \mathbb{Q}_{i^{\prime}} \frac{\partial \hat{w}^{i}}{\partial \hat{x}_{j}}\right) C_{i^{\prime} j^{\prime} k^{\prime} l^{\prime}}\left(\mathbb{Q}_{l l^{\prime}}^{-1} \mathbb{Q}_{k^{\prime} k} \frac{\partial \hat{v}^{k}}{\partial \hat{x}_{l}}\right) \mathrm{d} \hat{\Omega} \\
& =E_{1} \int_{\hat{\Omega}}\left(\mathbb{Q}_{j j^{\prime}}^{-1} \mathbb{Q}_{i^{\prime} i} \frac{\partial \hat{w}^{i}}{\partial \hat{x}_{j}}\right) \mathbb{Q}_{i^{\prime} i^{\prime \prime}} \mathbb{Q}_{j^{\prime} j^{\prime \prime}} \mathbb{Q}_{k^{\prime} k^{\prime \prime}} \mathbb{Q}_{l^{\prime} l^{\prime \prime}} \hat{C}_{i^{\prime \prime} j^{\prime \prime} k^{\prime \prime} l^{\prime \prime}}\left(\mathbb{Q}_{l l^{\prime}}^{-1} \mathbb{Q}_{k^{\prime} k} \frac{\partial \hat{v}^{k}}{\partial \hat{x}_{l}}\right) \mathrm{d} \hat{\Omega} \\
& =E_{1} \int_{\hat{\Omega}} \frac{\partial \hat{w}^{i}}{\partial \hat{x}_{j}}\left(\mathbb{Q}_{i^{\prime} i} \mathbb{Q}_{i^{\prime} i^{\prime \prime}}\right)\left(\mathbb{Q}_{j j^{\prime}}^{-1} \mathbb{Q}_{j^{\prime} j^{\prime \prime}}\right)\left(\mathbb{Q}_{k^{\prime} k} Q_{k^{\prime} k^{\prime \prime}}\right)\left(\mathbb{Q}_{l l^{\prime}}^{-1} \mathbb{Q}_{l^{\prime} l^{\prime \prime}}\right) \hat{C}_{i^{\prime \prime} j^{\prime \prime} k^{\prime \prime} l^{\prime \prime}} \frac{\partial \hat{v}^{k}}{\partial \hat{x}_{l}} \mathrm{~d} \hat{\Omega} \\
& =E_{1} \int_{\hat{\Omega}} \frac{\partial \hat{w}^{i}}{\partial \hat{x}_{j}}\left(\mathbb { Q } ^ { \mathrm { T } } \mathbb { Q } _ { i i ^ { \prime \prime } } \left(\mathbb { Q } ^ { - 1 } \mathbb { Q } _ { j j ^ { \prime \prime } } \left(\mathbb { Q } ^ { \mathrm { T } } \mathbb { Q } _ { k k ^ { \prime \prime } } \left(\mathbb{Q}^{-1} \mathbb{Q}_{l l^{\prime \prime}} \hat{C}_{i^{\prime \prime} j^{\prime \prime} k^{\prime \prime} l^{\prime \prime}} \frac{\partial \hat{v}^{k}}{\partial \hat{x}_{l}} \mathrm{~d} \hat{\Omega}\right.\right.\right.\right. \\
& =E_{1} \int_{\hat{\Omega}} \frac{\partial \hat{w}^{i}}{\partial \hat{x}_{j}} \hat{C}_{i j k l} \frac{\partial \hat{v}^{k}}{\partial \hat{x}_{l}} \mathrm{~d} \hat{\Omega} \\
& =\hat{a}_{1}(\hat{w}, \hat{v} ; \mu) .
\end{aligned}
$$


The key point in (163)-(169) is that the representation of the instantiated bilinear form in archetype coordinates does not require parameters related to the rotation Jacobian $\mathbb{Q}$ due to the cancellations in (167) and the fact that $\operatorname{det} \mathbb{Q}=1$. For our $R B$ approximations we employ the archetype domain for all computations and thus the RB spaces do not need to take these "docking" parameters into account. Furthermore the matrix and vector blocks for component instantiations that differ only in spatial orientation are identical, which thus contributes to the realization of $I_{\text {eff }} \ll I$ in an instantiated system.

We next consider our second archetype, component 2 , which is a short cylinder stub. It has two ports of type port 1 and may thus connect to the left and right ports of component 1 . The reference port space dimension is again $\mathcal{N}^{\beta}=219$. The FE discretization for component 2 has $\mathcal{N}_{2}=3,504$ degrees of freedom in linear hexahedral elements. The parameter vector for this component is

$$
\hat{\mu}_{2}=\left(\hat{E}_{2}, \hat{L}_{2}, \hat{\kappa}_{2, \text { left }}^{\mathrm{tr}}, \hat{\kappa}_{2, \text { right }}^{\mathrm{tr}}\right),
$$

where $\hat{E}_{2}=\hat{E}_{2}^{\mathrm{dim}} / \hat{E}_{2}^{\mathrm{dim}, 0}$ is a Young's modulus scaling parameter, $\hat{L}_{2}=\hat{L}_{2}^{\mathrm{dim}} / \hat{L}^{\mathrm{dim}, 0} \in$ $[0.5,2]$ is a length scaling parameter, and $\hat{\kappa}_{2 \text {,left }}^{\text {tr }}$ and $\hat{\kappa}_{2 \text {,right }}^{\text {tr }}$ are directional traction applied on the left and right ports, respectively. The archetype bilinear and linear forms associated with component 2 are, for all $\hat{w}, \hat{v} \in \hat{X}_{2}\left(\hat{\Omega}_{2}\right)$, given as

$$
\begin{aligned}
\hat{a}_{2}\left(\hat{w}, \hat{v} ; \hat{\mu}_{2}\right)= & \hat{E}_{2}\left(\int_{\hat{\Omega}_{2}} \frac{\partial \hat{w}^{i}}{\partial \hat{x}_{j}} \hat{C}_{i j k 3} \frac{\partial \hat{v}^{k}}{\partial \hat{x}_{3}}+\int_{\hat{\Omega}_{2}} \frac{\partial \hat{w}^{i}}{\partial \hat{x}_{3}} \hat{C}_{i 3 k l} \frac{\partial \hat{v}^{k}}{\partial \hat{x}_{l}}\right) \\
& +\frac{\hat{E}_{2}}{\hat{L}_{2}} \int_{\hat{\Omega}_{2}} \frac{\partial \hat{w}^{i}}{\partial \hat{x}_{3}} \hat{C}_{i 3 k 3} \frac{\partial \hat{v}^{k}}{\partial \hat{x}_{3}}+\hat{E}_{2} \hat{L}_{2} \int_{\hat{\Omega}_{2}} \frac{\partial \hat{w}^{i}}{\partial \hat{x}_{j}} \hat{C}_{i j k l} \frac{\partial \hat{v}^{k}}{\partial \hat{x}_{l}}, \\
\hat{f}_{2}\left(\hat{v} ; \hat{\mu}_{2}\right)= & \hat{\kappa}_{2, \text { left }, i}^{\operatorname{tr}} \int_{\hat{\gamma}_{2, \text { left }}} \hat{v}^{i}+\hat{\kappa}_{2, \text { right }, i}^{\operatorname{tr}} \int_{\hat{\gamma}_{2, \text { right }}} \hat{v}^{i},
\end{aligned}
$$

where, in (171), $j$ and $l$ take only the values 1,2 , and where, in (172) $\hat{\gamma}_{2 \text {,left }}$ and $\hat{\gamma}_{2 \text {,right }}$ are the boundaries associated with the left and right port, respectively. We note that $\hat{Q}_{2}^{a}=3$ and that $\hat{Q}_{2}^{f}=6$. Note that the bilinear form depends on the dilation parameter $\hat{L}_{2}$, but not on spatial orientation of the component; we may show this by reverse application of the arguments in (163)-(169) to each of three terms in (171). For the bound conditioner reference matrix blocks we consider three parameter values $\hat{\mu}_{2 \text {,ref }}^{1}=\left(\hat{E}_{2, \text { ref, }}^{1} \hat{L}_{2, \text { ref }}^{1}\right)=(1,0.75)$, $\hat{\mu}_{2, \text { ref }}^{2}=\left(\hat{E}_{2, \text { ref }}^{2}, \hat{L}_{2, \text { ref }}^{2}\right)=(1,1)$, and $\hat{\mu}_{2 \text {,ref }}^{3}=\left(\hat{E}_{2, \text { ref }}^{3} \hat{L}_{2, \text { ref }}^{3}\right)=(1,1.5)$ (thus $\left.n_{2, \text { ref }}=3\right)$.

\section{Pairwise empirical port mode training}

We now discuss the pairwise empirical port mode training for our library components. For the port 1 type we consider the three component pairs shown in

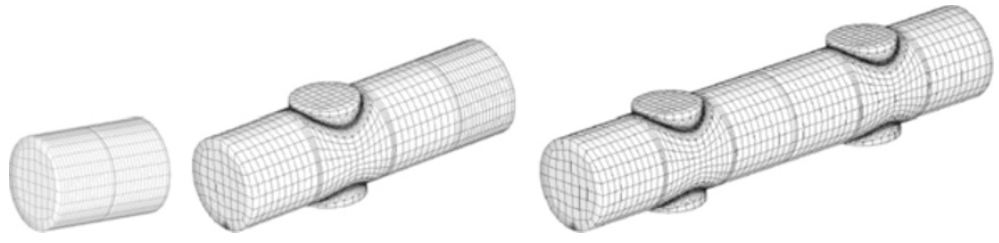

Figure 5 Component pairs used for empirical training of port 1 . 
Figure 5: a pair with two instantiations of component 2, a pair with one instantiation of component 1 and one instantiation of component 2, and a pair with two instantiations of component 1 connected via side ports (port 1 ). For the port 2 type we consider the single pair of instantiations of component 1 connected via a port 2 type port as shown in Figure 6; note that when we consider a large microtruss structure we shall always "weld" our cylinders in this particular cross configuration.

We then execute Algorithm 1 for each pair; for the boundary condition regularity parameter in Algorithm 1 we choose $\gamma=3$. For the training of port 1 we extract $N_{\text {samples }}=150$ different port samples in $S_{\text {pair }}$ from each of the three pairs; recall that we subtract the projection onto the six modes $\hat{\chi}_{i}^{\beta}, 1 \leq i \leq 6$, related to rigid-body motion from all snapshots. We then combine all 450 modes in $S_{\text {type }}$, and perform a POD over these 450 modes to compress the data to $n_{\text {pod }}^{\beta}=44$ POD modes. We then obtain $n_{\text {pod }}^{\beta}+6=50$ empirical modes, which we complement by $\mathcal{N}^{\beta}-50=169$ eigenmodes (restricted to the orthogonal complement space) to complete the discrete space (note in practice we shall always use less than 50 modes for the port-reduced SCRBE approximation).

The approach for the training of port 2 is identical except we perform POD over $N_{\text {samples }}=300$ different port samples (with the projections onto the rigid body modes subtracted) extracted from the single component pair. We choose the same number of POD modes $\left(n_{\mathrm{POD}}^{\beta}=44\right)$ and thus empirical modes for this port type.

We shall use these empirical port modes for most of our numerical results below. However we shall also compare these results to results obtained using more standard (and in

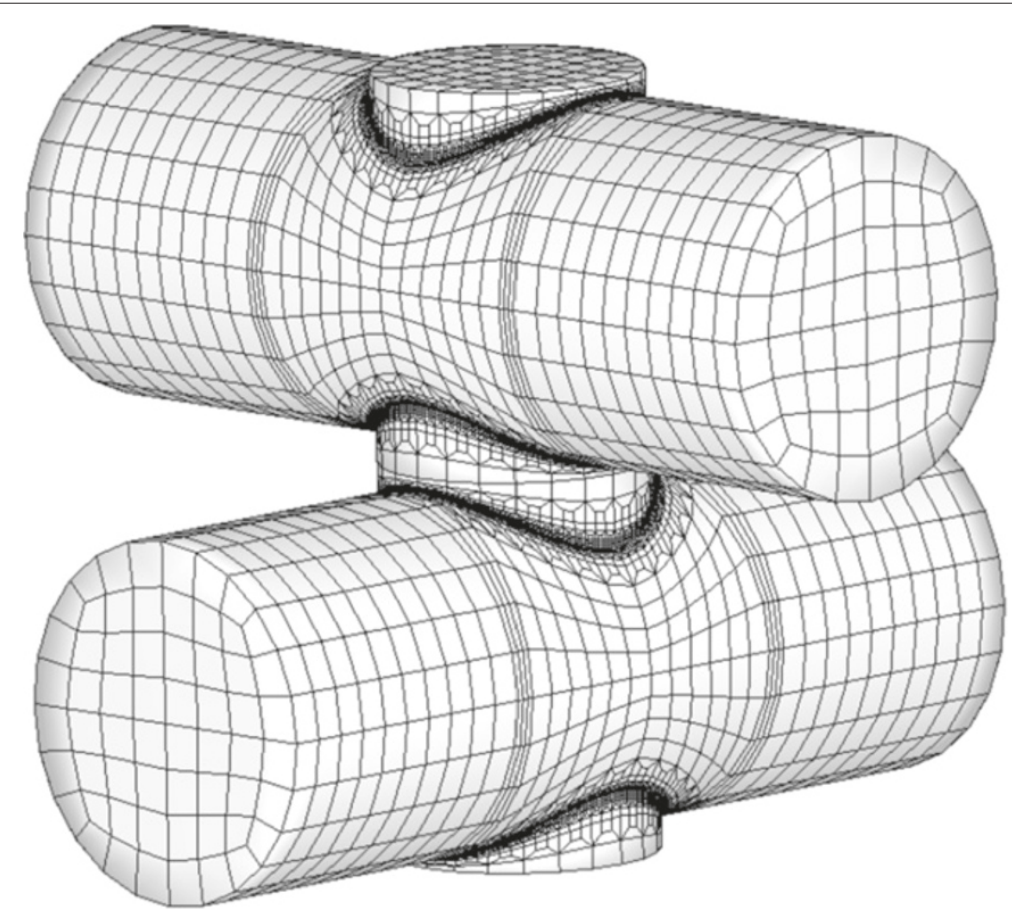

Figure 6 Component pairs used for empirical training of port 2 . 
particular non-empirical) "Legendre" port eigenmodes. In this latter case, the reference port modes $\hat{\chi}_{k}^{\beta}$ are given as

$$
\begin{aligned}
\hat{\chi}_{1}^{\beta}=\left(L_{1}^{\beta}, 0,0\right), \hat{\chi}_{2}^{\beta}=\left(0, L_{1}^{\beta}, 0\right), \hat{\chi}_{3}^{\beta}= & \left(0,0, L_{1}^{\beta}\right), \\
& \hat{\chi}_{4}^{\beta}=\left(L_{2}^{\beta}, 0,0\right), \hat{\chi}_{5}^{\beta}=\left(0, L_{2}^{\beta}, 0\right), \hat{\chi}_{6}^{\beta}=\left(0,0, L_{2}^{\beta}\right), \ldots
\end{aligned}
$$

where the $L_{i}^{\beta}, 1 \leq i \leq \mathcal{N}^{\beta} / 3$, are the eigenvectors of a scalar singular Sturm-Liouville eigenproblem over $\beta$ ordered according to increasing eigenvalue.

\section{Numerical results}

We now present numerical results for our three-dimensional linear-elastic microtruss library to demonstrate our port-reduced SCRBE approximation and error estimation framework. Our implementation is in $\mathrm{C}++$ and is based on the library libMesh [24,25]. In our current implementation offline calculations are performed in parallel, while online calculations are limited to a single core.

The offline computation time for our microtruss library is about five hours using up to 24-cores on an AMD Opteron 6238 workstation computer. In offline stages Off 4 and Off7 we load all required data into memory to prepare for the online stage. An (upper bound for) the online memory footprint for this library is $1.5 \mathrm{~Gb}$.

\section{Cylindrical cantilever beam}

We shall first consider a cylindrical cantilever beam system, for which we may compare our compliance output results to standard (Euler-Bernoulli) beam theory [26]. Hence this system provides an opportunity to confirm both the validity of the SCRBE framework in terms both of approximation and certification - as well as the fidelity of the underlying FE "truth" component discretization.

Our cantilever system is of total length $l=8 L$ and consists of $I=8$ instantiations of component 2 of individual length $L_{i}=L, 1 \leq i \leq I$; we consider $E_{i}=1,1 \leq$ $i \leq I$, and thus here $I_{\text {eff }}=1$. We prescribe zero Dirichlet conditions on the left-most port of the system and we apply a unity-magnitude tangential traction on the right-most port as shown in Figure 7; the deformations in Figure 7 show the displacement field, and the colors indicate the Von Mises stresses ${ }^{\mathrm{i}}$ with higher stresses in red. The output for this system is the average displacement over the right-most port in the direction of the

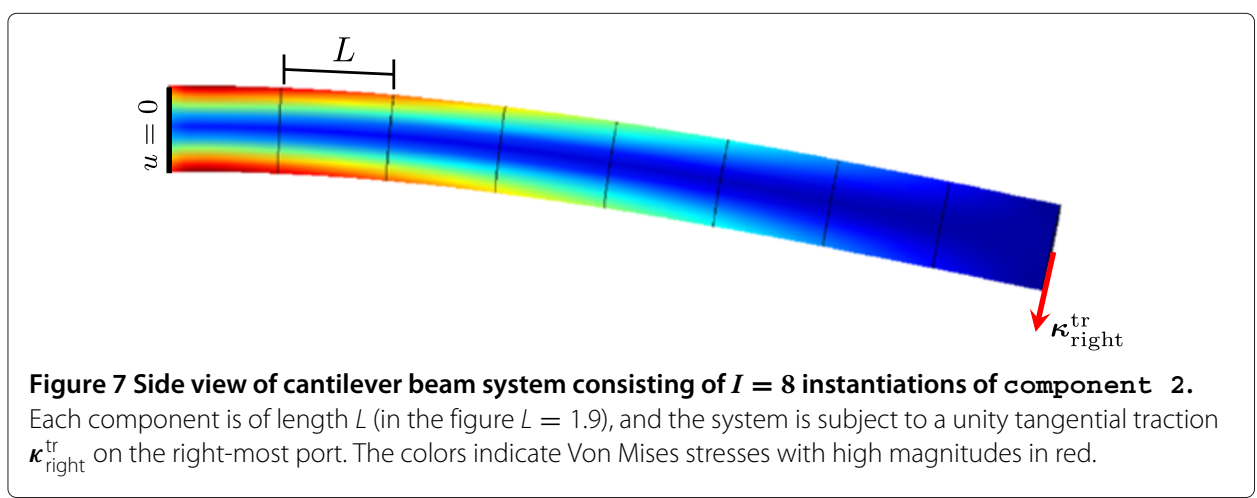


specified traction and is thus equal to compliance normalized by port area. We use $n_{\mathrm{A}, p}^{\Gamma}=$ 20 empirical port modes on all global ports $\Gamma_{p}, 1 \leq p \leq n^{\Gamma}$.

We report in the second, third, and fourth columns of Table 1 the port-reduced SCRBE compliance output approximation $\tilde{s}^{\mathrm{PR}}(\mu)$, the relative error in $\tilde{s}^{\mathrm{PR}}(\mu)$ with respect to the FE "truth" compliance output $s^{h}(\mu)$, and the effectivity of the compliance output estimator, $\Delta^{s ; 0}(\mu)$, respectively. We note that the error in the output approximation is very small, and that the error estimator is relatively sharp; note that for large values of $L$ the term $\left|\tilde{\mathbb{U}}^{\mathrm{PR}}(\mu)\right|^{\mathrm{T}} \boldsymbol{\sigma}_{\mathbb{A}}(\mu)\left|\tilde{\mathbb{U}}^{\mathrm{PR}}(\mu)\right|$ dominates in (136) and causes the effectivity to increase somewhat. We emphasize that our error estimator is for all these cases indeed an error upper bound: the effectivities are greater than unity.

We report in the fourth and fifth columns of Table 1 the theoretical maximum deflection $s^{\mathrm{EB}}(\mu)$ as predicted by classical Euler-Bernoulli beam theory, ${ }^{\mathrm{j}}$ and the relative difference between $\tilde{s}^{\mathrm{PR}}(\mu)$ and $s^{\mathrm{EB}}(\mu)$. The theoretical predictions match the computational results reasonably well, and in particular become increasingly accurate for larger $L$ (the analytical results are valid in the limit of a long cantilever). Furthermore the discrepancy is for larger $L$ sufficiently small that we deem our component FE discretization sufficiently rich.

Next, we consider the behavior of our port-reduced SCRBE compliance output approximation and associated error estimator as functions of $n_{\mathrm{A}, p}^{\Gamma}$ empirical port modes for a fixed length parameter $L=1.3$. In Figure 8 we report the relative compliance error $\left|s^{h}(\mu)-\tilde{s}^{\mathrm{PR}}(\mu)\right| / \tilde{s}^{\mathrm{PR}}(\mu)$, the relative error estimator $\Delta^{s ; 0}(\mu) / \tilde{s}^{\mathrm{PR}}(\mu)$ given in (149), and the relative error bound $\Delta^{s}(\mu ; C) / \tilde{s}^{\mathrm{PR}}(\mu)$ given in Proposition 3 realized for $C=$ $1 / \lambda_{\min }\left(\mathbb{B}_{\mu}^{N C}\right)$. We make several observations: first, the relative error decreases very fast and is of order $10^{-4}$ already for $n_{\mathrm{A}, p}^{\Gamma}=10$. Second, the error estimator is always greater than the error and is furthermore reasonably sharp - the effectivity is $\mathcal{O}(10)-$ for $n_{\mathrm{A}, p}^{\Gamma} \leq 18$; at $n_{\mathrm{A}, p}^{\Gamma}=18$ the $\mathrm{RB}$ error bound contribution $|\tilde{\mathbb{U}}(\mu)|^{\mathrm{T}} \boldsymbol{\sigma}_{\mathbb{A}}(\mu)|\tilde{\mathbb{U}}(\mu)|$ becomes the dominating term in (136) and thus adding additional port modes will not reduce the error estimator. ${ }^{k}$ Third, the rigorous error bound is reasonably sharp only for small $n_{\mathrm{A}, p}^{\Gamma}$ : the term $\|\sigma(\mu)\|_{2}^{2} / \lambda_{\min }\left(\mathbb{B}_{\mu}^{N C}\right)$ in (128) dominates from an early point not because of large RB error bound contributions per se but because $C=$ $1 / \lambda_{\min }\left(\mathbb{B}_{\mu}^{\mathrm{NC}}\right)$ is a pessimistic estimate for the Rayleigh quotient associated with $\mathbb{B}_{\mu}^{\mathrm{NC}}$ and $\sigma(\mu)$.

We also compare our empirical port approximation to the more standard eigenmode (Legendre) port approximation introduced in (173). In Figure 9 we report for the

Table 1 Results for variable $L$ for the cylindrical cantilever beam system using $n_{\mathrm{A}, p}^{\Gamma}=20$ empirical port modes on each port

\begin{tabular}{llcccc}
\hline $\boldsymbol{l}=\mathbf{8} \boldsymbol{L}$ & $\tilde{\boldsymbol{s}}^{\mathbf{P R}}(\boldsymbol{\mu})$ & $\frac{\boldsymbol{s}^{\boldsymbol{h}}(\boldsymbol{\mu})-\tilde{\boldsymbol{s}}^{\mathbf{P R}}(\boldsymbol{\mu})}{\tilde{\boldsymbol{s}}^{\mathbf{P R}}(\boldsymbol{\mu})}$ & $\frac{\boldsymbol{\Delta}^{\boldsymbol{s} \mathbf{0}}(\boldsymbol{\mu})}{\left|\boldsymbol{s}^{\boldsymbol{h}}(\boldsymbol{\mu})-\tilde{\boldsymbol{s}}^{\mathbf{P R}}(\boldsymbol{\mu})\right|}$ & $\boldsymbol{s}^{\mathrm{EB}}(\boldsymbol{\mu})$ & $\frac{\tilde{\boldsymbol{s}}^{\boldsymbol{P R}}(\boldsymbol{\mu})-\boldsymbol{s}^{\boldsymbol{E B}}(\boldsymbol{\mu})}{\tilde{\boldsymbol{s}}^{\mathbf{P R}}(\boldsymbol{\mu})}$ \\
\hline 4.0 & $1.6504 \mathrm{e}+2$ & $8.4 \mathrm{e}-5$ & $1.7 \mathrm{e}+1$ & $1.5562 \mathrm{e}+2$ & $5.7 \mathrm{e}-2$ \\
5.6 & $3.8 \mathrm{e}-5$ & $6.8 \mathrm{e}+0$ & $4.2702 \mathrm{e}+2$ & $2.8 \mathrm{e}-2$ \\
7.2 & $9.2369 \mathrm{e}+2$ & $2.8 \mathrm{e}-5$ & $2.3 \mathrm{e}+1$ & $9.0758 \mathrm{e}+2$ & $1.7 \mathrm{e}-3$ \\
8.8 & $1.6767 \mathrm{e}+3$ & $2.4 \mathrm{e}-5$ & $4.5 \mathrm{e}+1$ & $1.6571 \mathrm{e}+3$ & $1.2 \mathrm{e}-3$ \\
10.4 & $2.7584 \mathrm{e}+3$ & $2.2 \mathrm{e}-5$ & $3.3 \mathrm{e}+1$ & $2.7352 \mathrm{e}+3$ & $8.4 \mathrm{e}-3$ \\
12.0 & $4.2281 \mathrm{e}+3$ & $1.8 \mathrm{e}-5$ & $2.5 \mathrm{e}+1$ & $4.2018 \mathrm{e}+3$ & $6.2 \mathrm{e}-3$ \\
13.6 & $6.1450 \mathrm{e}+3$ & $1.3 \mathrm{e}-5$ & $1.7 \mathrm{e}+2$ & $6.1165 \mathrm{e}+3$ & $4.6 \mathrm{e}-3$ \\
15.2 & $8.5671 \mathrm{e}+3$ & $2.0 \mathrm{e}-5$ & $3.5 \mathrm{e}+2$ & $8.5392 \mathrm{e}+3$ & $3.3 \mathrm{e}-3$ \\
\hline
\end{tabular}




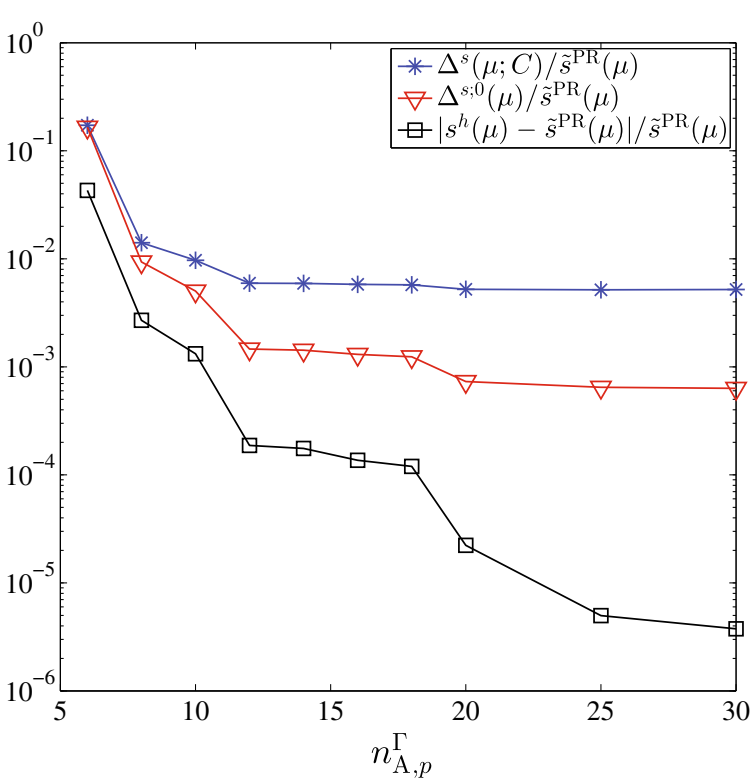

Figure 8 Relative compliance output error, relative output error estimator, and relative output error bound as functions of $n_{A}^{\Gamma}$ for the cantilever beam $(L=1.3)$, using empirical port spaces.

Legendre case the relative compliance error, the relative error estimator, and the relative error bound superposed on the results for the empirical case (in gray). From the two error curves (squares) we note that the empirical port mode approximation is more than an order of magnitude better than the Legendre approximation for small $n_{\mathrm{A}, p}^{\Gamma}$, and for larger $n_{\mathrm{A}, p}^{\Gamma}$ the error in the Legendre approximation decreases significantly only for certain eigenmodes whereas the empirical approximation converges in a more regular

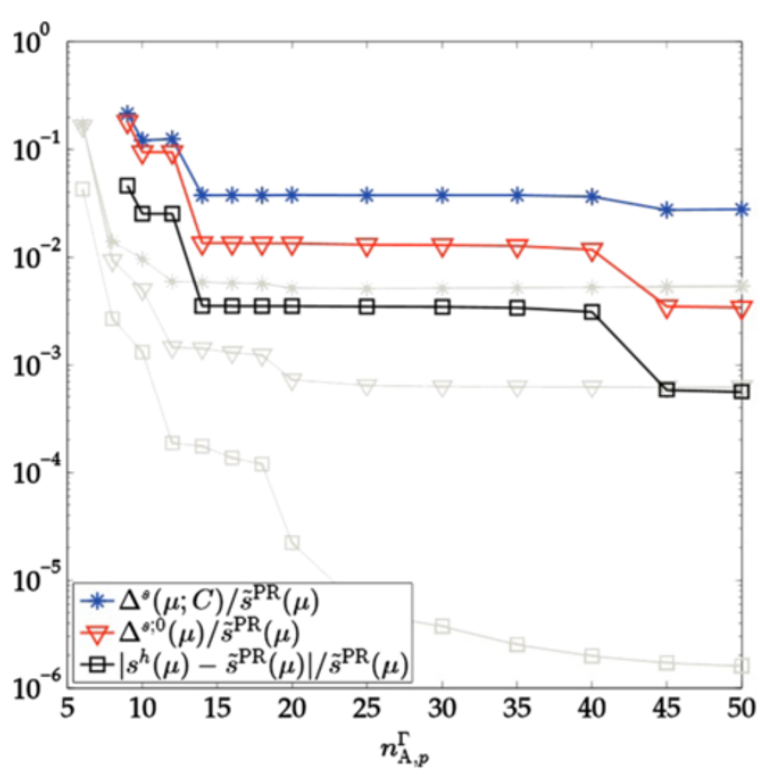

Figure 9 Relative compliance output error, relative output error estimator, and relative output error bound as functions of $n_{A}^{\Gamma}$ for the cantilever beam $(L=1.3)$, using Legendre port spaces (superposed on the results for empirical port spaces). 
fashion. We also note that the error estimator (triangles) and bound (asterisk) for the empirical approximation is about an order of magnitude smaller than the estimator and bound for the Legendre approximation, respectively.

Finally, we consider in Figure 10 and Figure 11 the relative compliance output error estimator $\Delta^{s ; 0}(\mu) / \tilde{s}^{\mathrm{PR}}(\mu)$ for empirical and Legendre port modes as functions of $L$ for three different values of $n_{\mathrm{A}, p}^{\Gamma}$; note the results for the latter case are superposed on the results for the former case in Figure 11. We note that for $L$ far from the reference parameter values (recall $\hat{L}_{2 \text {,ref }}^{1}=0.75, \hat{L}_{2 \text {,ref }}^{2}=1.0, \hat{L}_{2 \text {,ref }}^{3}=1.5$ ) only the empirical port modes provide a good approximation; in fact using empirical port modes we obtain even for $n_{\mathrm{A}, p}^{\Gamma}=10 \mathrm{a}$ relative error estimator smaller than 0.013 for all sampled values of $L$. Again, we emphasize that these error estimates indeed provide bounds on the error: for all cases the relative error with respect to the FE discretization is smaller than $10^{-4}$ as reported in the second column of Table 1.

For the remainder of our numerical results we exclusively employ the error estimator (136) or (148) rather than the respective rigorous bound.

\section{Microtruss structure}

We shall now consider a larger microtruss beam. Our first microtruss system, system 1 , is an array of of $I=408$ components (224 of which are of archetype component 1 and 184 of which are of archetype component 2). We illustrate the system assembly process in Figure 12 and Figure 3; note that this procedure is efficient thanks to a graphical user interface that allows "cloning" of smaller subsystems which we may interconnect to form the final system [Additional file 1]. Note in actual (engineering) practice, this microtruss beam may be manufactured from $N_{\text {rods }}=40$ rods that are welded together.

To the final system shown in Figure 3 we apply zero Dirichlet boundary conditions on the 32 bottom ports; we apply homogeneous Neumann boundary conditions on the 80 side ports; we apply a unity-magnitude tangential traction (Neumann) $\boldsymbol{\kappa}_{\text {top }}^{\mathrm{tr}}$ on the 32 top (red) ports in the $z$-direction. The size of the non-port-reduced Schur complement system

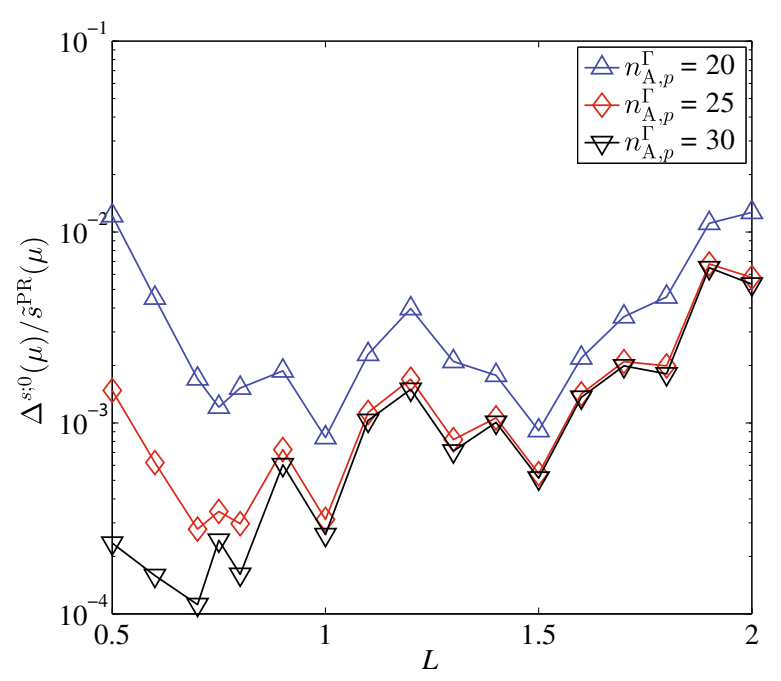

Figure 10 Cantilever beam relative compliance error estimator as a function of $L$ for different $n_{A, p}^{\Gamma}$ using empirical port spaces. 


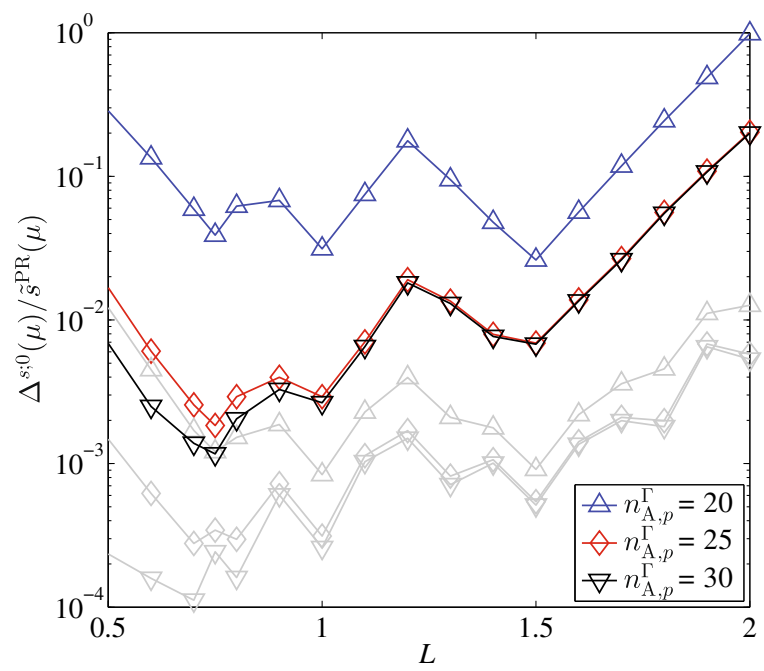

Figure 11 Cantilever beam relative compliance error estimator as a function of $L$ for different $n_{\mathrm{A}, p}^{\Gamma}$ using Legendre port spaces (superposed on the results for empirical port spaces).

is in this case $n_{\mathrm{SC}}=147,168$. Note that system 1 refers to a particular topology configured with particular Dirichlet boundary conditions; we shall thus consider system 1 for many different system parameter values. In particular, we denote by $L^{z}$ and $L^{x}$ the length of all component instantiations of component 2 which are oriented in the $z$-direction and $x$-direction, respectively, as indicated for $L^{z}$ in Figure 3.

We first demonstrate the ability of the port-reduced SCRBE framework to provide FE-fidelity field approximations at low computational cost. For our first calculation we consider the parameter values $E_{i}=1,1 \leq i \leq I, L^{z}=1.1$, and $L^{x}=1$. We show (qualitatively) the solution fields in Figure 13 and Figure 14: the displacement field is shown in Figure 13 as a deformation of the original geometry (compare to the original geometry in Figure 3); a closeup of the Von Mises stress field near a "weld" is shown in Figure 14. The high-stress concentrations (red) are typically isolated to areas where a weld meets the cylinder base. Note that this high-stress, near-singular, area of the field is located somewhat close to the ports but nevertheless well within the interior of the
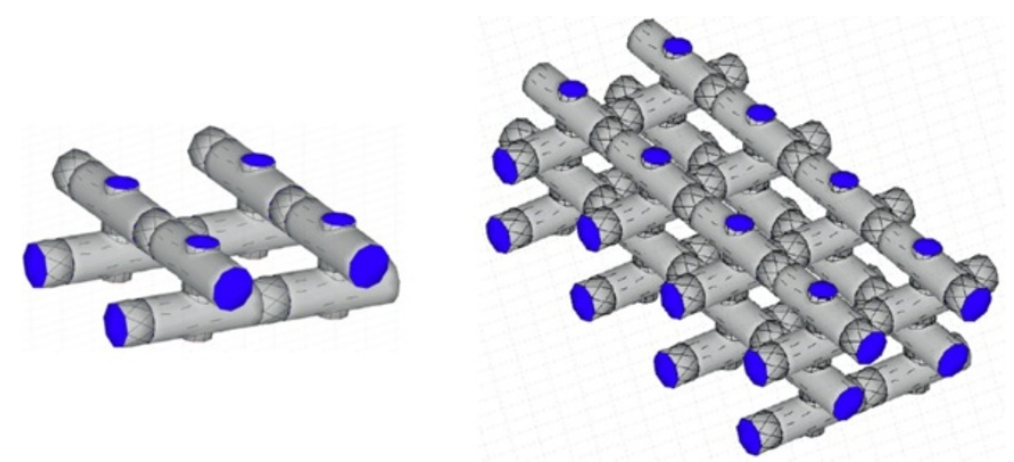

Figure 12 Assembly of the microtruss structure by component and subsystem "cloning" using a graphical user interface. 


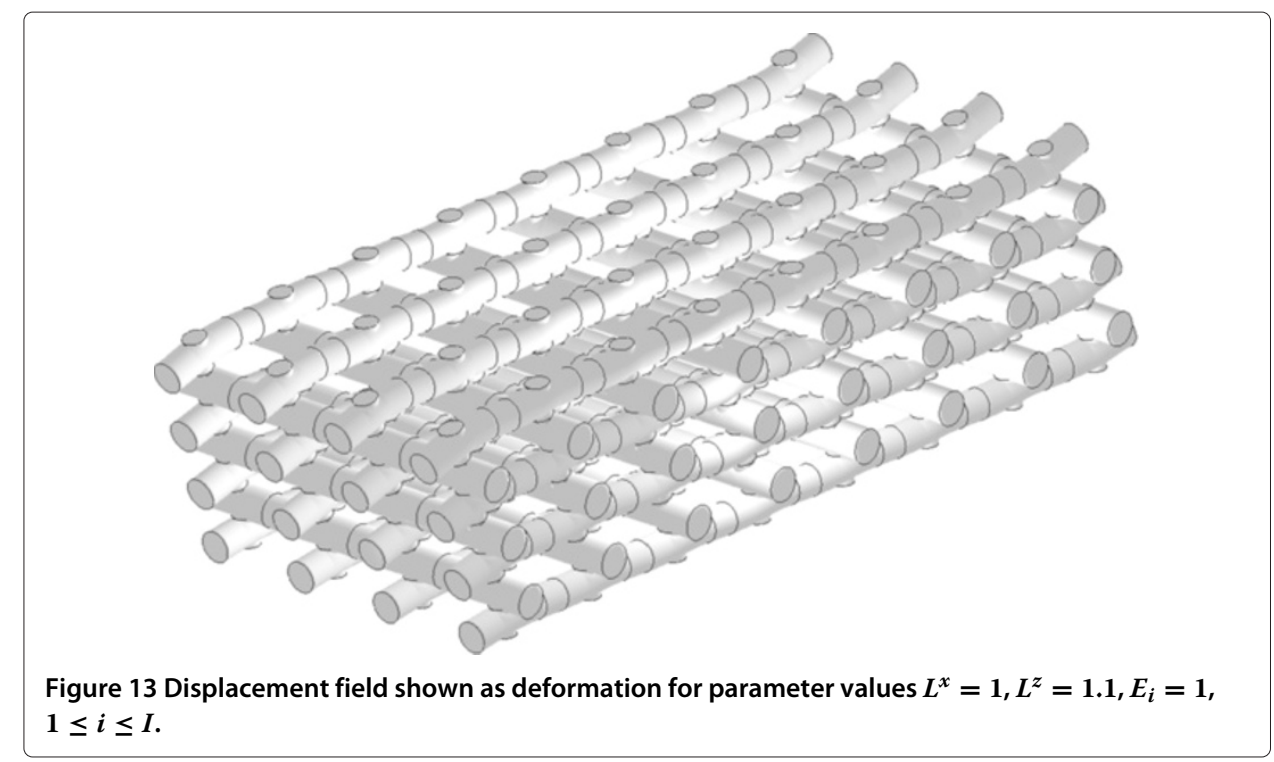

components. Placement of singular or more rapid behavior within the interior of a component, when possible, can reduce the number of port degrees of freedom required as provided by the pairwise training algorithm.

For $n_{\mathrm{A}, p}^{\Gamma}=20$ and $n_{\mathrm{A}, p}^{\Gamma}=25$ "Active" port modes we obtain the relative energy-norm error estimators

$$
\frac{\left\|e^{h}(\mu)\right\|_{\mu}}{\left\|\tilde{u}^{\mathrm{PR}}(\mu)\right\|_{\mu}} \leq \frac{\Delta^{u ; 0}(\mu)}{\left\|\tilde{u}^{\mathrm{PR}}(\mu)\right\|_{\mu}}=0.1139, \quad \frac{\left\|e^{h}(\mu)\right\|_{\mu}}{\left\|\tilde{u}^{\mathrm{PR}}(\mu)\right\|_{\mu}} \leq \frac{\Delta^{u ; 0}(\mu)}{\left\|\tilde{u}^{\mathrm{PR}}(\mu)\right\|_{\mu}}=0.05641
$$

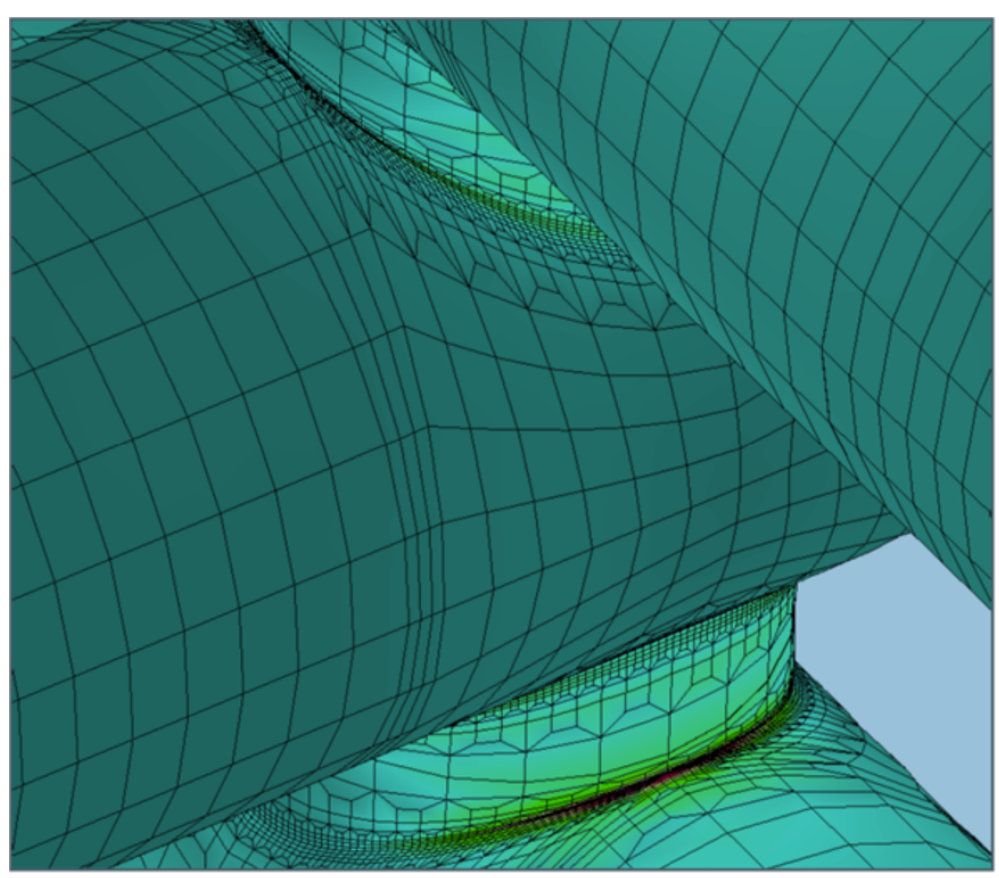

Figure 14 Von Mises stress field for parameter values $L^{x}=1, L^{z}=1.1, E_{i}=1,1 \leq i \leq I$. 
respectively (note the inequalities are not confirmed but valid under the assumption that the error estimators indeed provide error bounds). The port-reduced SCRBE system sizes are $n_{\mathrm{A}}=13,440$ and $n_{\mathrm{A}}=16,800$, respectively, and we thus realize in both cases $n_{\mathrm{A}} \ll n_{\mathrm{SC}}$. For these calculations $I_{\text {eff }}=4-$ there are only two unique instantiations of component $1^{1}$ and only two unique instantiations of component $2-$ and we thus realize very efficient online computations. The total (for solution and error estimate) online CPU time is approximately 12.9 seconds for the $n_{\mathrm{A}, p}^{\Gamma}=20$ calculation and approximately 18 seconds for the $n_{\mathrm{A}, p}^{\Gamma}=25$ calculation. We report detailed online timing results in the left and middle columns of Table 2, and we note that for both computations the certification dominates online cost. In particular, the calculation of the minimum eigenvalue (On4) together with the non-conforming solves (On7) contribute roughly $8 / 10$ of total cost. Note that as we consider the error estimator rather than the error bound, we do not execute On8.

We next demonstrate the ability of the SCRBE framework to handle different topological configurations, here in the form of a simulated material flaw. To this end, we introduce a second microtruss system, system 2, which is identical to system 1 except we assume that three "random" welds are broken such that now we do not couple the corresponding port 2 ports (top or bottom local ports of component 1 ). These three shared global ports are thus split into six non-shared global ports, on which we impose homogeneous Neumann (zero-stress) boundary conditions. On all other ports the boundary conditions are the same as for system 1 . The size of the non-port-reduced Schur complement system is in this case slightly larger: the non-port-reduced system is of size $n_{\mathrm{SC}}=147,825$, and the port-reduced system for $n_{\mathrm{A}, p}^{\Gamma}=20$ active port modes is of size $n_{\mathrm{A}}=13,500$. A closeup of the solution field near a broken weld is shown in Figure 15; note the low stress concentration at the failed weld compared to neighboring intact welds.

We now consider the compliance output and associated error estimators for system 1 and system 2 . The compliance is for these systems the integrated displacement in the $z$-direction over all top (red in Figure 3 ) ports, and is thus effectively a measure of the microtruss beam directional stiffness. For system 1 and system 2 we then compute solutions and corresponding compliance outputs for different values of $L^{z} \in[0.5,2]$; we consider $L_{x}=1$ and $E_{i}=1,1 \leq i \leq I$. We thus effectively consider the directional stiffness of the microtruss beams as a function of the spacing between rods oriented in the $x$-direction.

The results for $n_{\mathrm{A}, p}^{\Gamma}=20$ are shown in Figure 16. The solid blue and solid red lines indicate the port-reduced SCRBE system 1 and system 2 output approximation, respectively; the dashed lines indicate the estimated bounds on the output as provided by

Table 2 Breakdown of majority of online computational cost in seconds for system 1 for indicated $n_{\mathrm{A}, p}^{\Gamma}$ active port modes and $I_{\text {eff }}$ unique component instantiations

\begin{tabular}{lccc}
\hline & $\boldsymbol{I}_{\boldsymbol{e f f}}=\mathbf{4}, \boldsymbol{n}_{\mathbf{A}, \boldsymbol{p}}^{\boldsymbol{\Gamma}}=\mathbf{2 0}$ & $\boldsymbol{I}_{\boldsymbol{e f f}}=\mathbf{4}, \boldsymbol{n}_{\mathbf{A}, \boldsymbol{p}}^{\Gamma}=\mathbf{2 5}$ & $\boldsymbol{I}_{\text {eff }}=\mathbf{8 0 ,} \boldsymbol{n}_{\mathbf{A}, \boldsymbol{p}}^{\boldsymbol{\Gamma}}=\mathbf{2 5}$ \\
\hline On2 & 0.5 & 0.6 & 8.3 \\
On3 & 1.3 & 2.5 & 2.5 \\
On4 & 6.5 & 7.8 & 9.2 \\
On5 & 0.8 & 0.9 & 18.1 \\
On7 & 3.8 & 6.2 & 6.2 \\
\hline Total & 12.9 & 18 & 44.3 \\
\hline
\end{tabular}




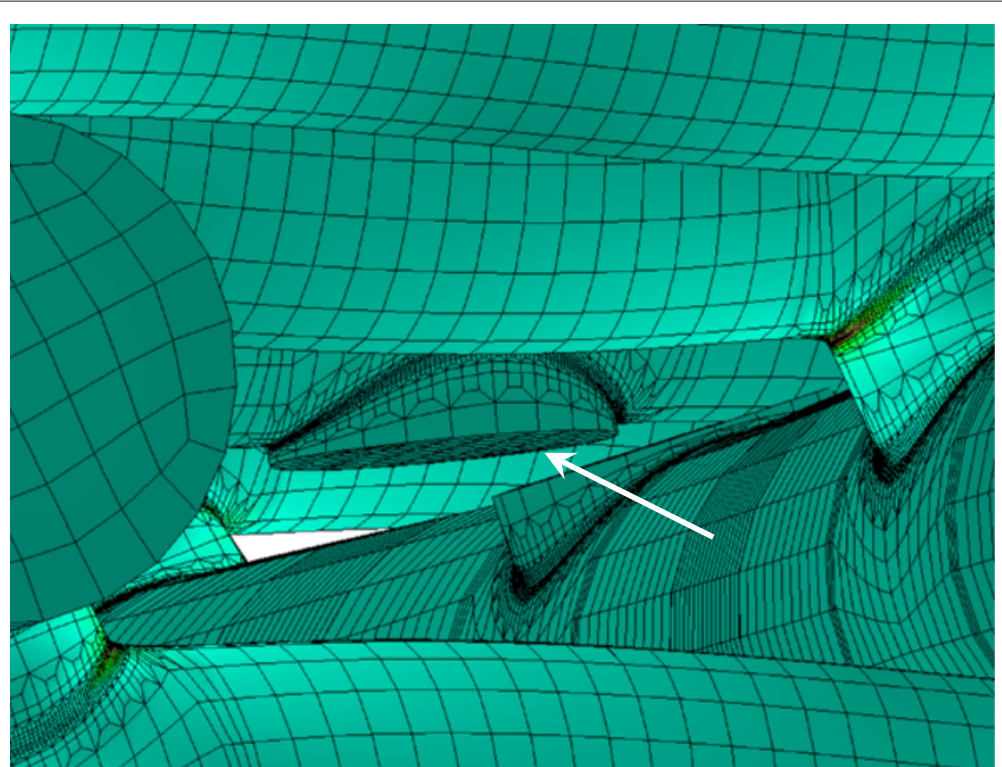

Figure 15 Solution (displacement and Von Mises stress (colors)) near a failed weld for system 2 .

the error estimator (149). As expected, system 2 (with the weld failure) is less stiff and thus exhibits larger top-port displacements than system 1 . However, we can not in this case for larger $L^{z}$ distinguish between system 1 and system 2 with any confidence because the (estimated) output bounds overlap.

We next consider the same "parameter sweep," but now using $n_{\mathrm{A}, p}^{\Gamma}=25$ empirical port modes. For system 1 this corresponds to a port-reduced SCRBE system of size $n_{\mathrm{A}}=$ 16,800 and for system 2 a system of size $n_{\mathrm{A}}=16,875$. In Figure 17, we show the outputs and output bounds for system 1 and system 2 , and we note that we are now able to easily distinguish the two systems.

So far we have for system 1 and system 2 considered only a single system parameter $L_{\mathrm{z}}$ and thus $I_{\text {eff }} \ll I$. We now consider for system 1 a somewhat more demanding case in which we also assign "random" Young's modulus $E_{\text {rod }}^{j} \in[0.9,1.1], 1 \leq j \leq N_{\text {rods }}$, to

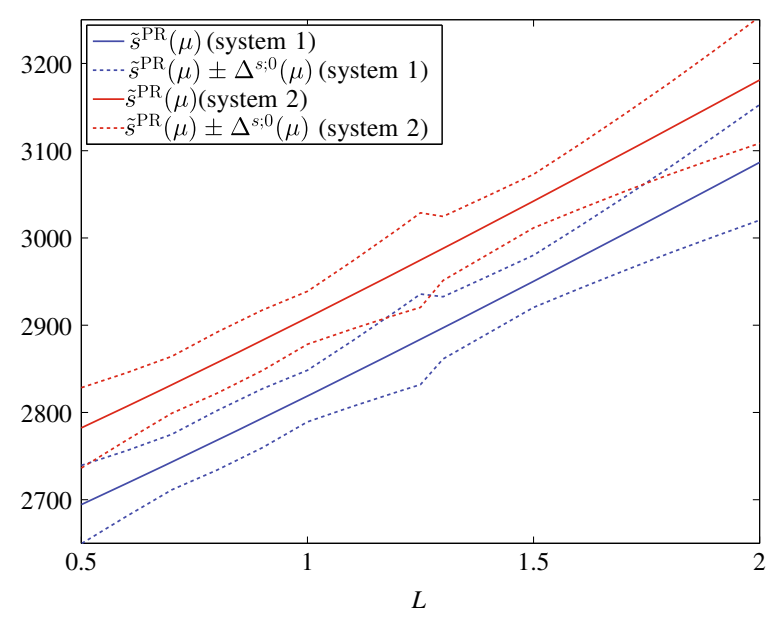

Figure 16 Parameter sweeps - compliance outputs and (estimated) compliance output bounds over $L^{z} \in[0.5,2]$ for system 1 and system 2 using $n_{\mathrm{A}, p}^{\Gamma}=20$ "Active" port modes. 


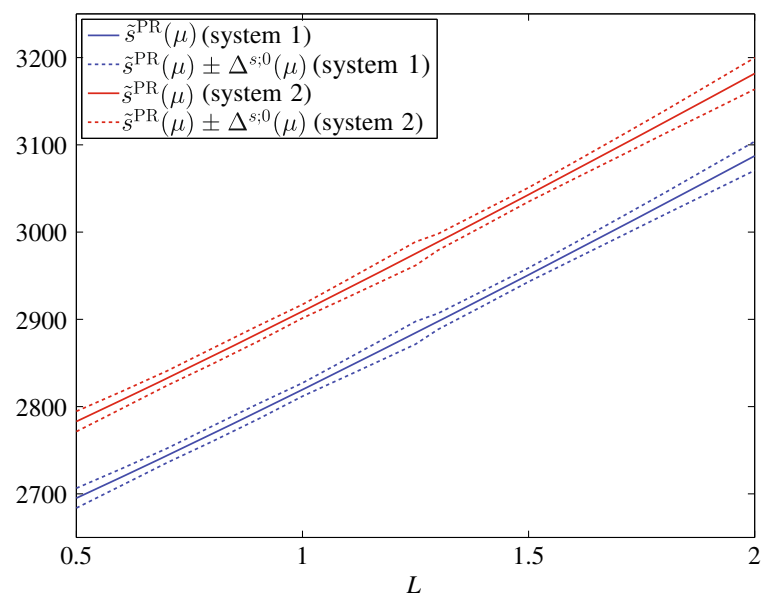

Figure 17 Parameter sweeps - compliance outputs and (estimated) compliance output bounds over $L^{z} \in[0.5,2]$ for system 1 and system 2 using $n_{A, p}^{\Gamma}=25$ "Active" port modes.

each of the $N_{\text {rods }}=40$ rods of the system; we thus consider here $P=40$ system parameters (one of the $E_{\text {rod }}^{j}$ scales out) and we obtain in this case $I_{\text {eff }}=80$. Because of the larger $I_{\text {eff }}$, computational cost increases somewhat as reported in the rightmost column of Table 2. ${ }^{\mathrm{m}}$ For this particular simulation we obtain a relative error field energy estimator $\Delta^{u ; 0}(\mu) /\left\|\tilde{u}^{\mathrm{PR}}(\mu)\right\|_{\mu}=0.0712$ using $n_{\mathrm{A}, p}^{\Gamma}=25$ empirical port modes.

Finally, we close this section with comparison to standard global FE analysis of system 1 for system parameters $L^{x}=1, L^{z}=1.1$ and $E_{i}=1,1 \leq i \leq I$. We consider $n_{\mathrm{A}, p}^{\Gamma}=20, n_{\mathrm{A}, p}^{\Gamma}=25$, and $n_{\mathrm{A}, p}^{\Gamma}=30$, and we report in Table 3 for each case the relative output error, $\left(s^{h}(\mu)-\tilde{s}^{\mathrm{PR}}(\mu)\right) / \tilde{s}^{\mathrm{PR}}(\mu)$, and the output error estimator effectivity, $\Delta^{s ; 0}(\mu) /\left(s^{h}(\mu)-\tilde{s}^{\mathrm{PR}}(\mu)\right)$. For all calculations the relative output error is indeed small, and certainly within acceptable tolerances in an engineering context. The estimator effectivities are furthermore greater than unity — our error estimators are indeed error upper bounds - and moreover, the efficivities show that our estimators are relatively sharp.

The global FE space $X^{h}(\Omega)$ for system 1 is of dimension $\mathcal{N}_{\mathrm{FE}}=26,381,328$. The computation time for a single global FE simulation on a workstation with eight AMD Opteron 6238 cores is 59 minutes for 93 conjugate gradient iterations using an algebraic multigrid preconditioner [27] (we employ the BoomerAMG [28] parallel algebraic multigrid implementation provided by the hypre [29] linear solver library). For $n_{\mathrm{A}, p}^{\Gamma}=25$, the portreduced SCRBE approximation and error estimation requires about 18 seconds on a single core and we thus obtain a speedup of almost 200 - note that significantly larger speedup is possible through parallelization of the SCRBE online stage. We finally note that to compute the results in Figure 17, we have for each parameter sweep performed seventeen evaluations of the port-reduced SCRBE compliance output and associated output bound

Table 3 Relative output error and output error estimator effectivity for sys tem 1 for parameter values $L^{x}=1, L^{z}=1.1$ and $E_{i}=1,1 \leq i \leq I$

\begin{tabular}{|c|c|c|c|}
\hline & $n_{\mathrm{A}, p}^{\Gamma}=20$ & $n_{\mathrm{A}, p}^{\Gamma}=25$ & $n_{A, p}^{\Gamma}=35$ \\
\hline$\frac{s^{h}(\mu)-\tilde{s}^{P R}(\mu)}{\tilde{s}^{P R}(\mu)}$ & $4.67978 \mathrm{e}-4$ & $2.31664 \mathrm{e}-4$ & $2.5646 e-5$ \\
\hline$\frac{\Delta^{s ; 0}(\mu)}{S^{h}(\mu)-\tilde{s}^{P R}(\mu)}$ & 28.3 & 14.8 & 67.6 \\
\hline
\end{tabular}


estimators in only about five minutes total CPU time. A similar parametric analysis using a classical FE approach is clearly not equally tractable.

\section{Conclusions}

In this paper we have extended the port-reduced static condensation reduced basis element method to analysis of large-scale component-based structures. In particular we have demonstrated the applicability and efficacy of the procedure in three-dimensional linear elasticity analysis of a microtruss structure with hundreds of components.

Through a combination of $i$ ) component-interior reduced basis approximations and ii) port reduction using empirical modes tailored to the component library, we are able to obtain an accurate online approximation for any component parameter values and any system topology using very few global degrees of freedom. Moreover, we may estimate (and rigorously bound in the limit of small reduced basis error contributions) the error in this port-reduced SCRBE approximation with respect to the underlying global finite element discretization through efficiently computable a posteriori errorl estimators.

For the microtruss application we consider in this paper, more than twenty-six million degrees of freedom in the alternative global FE discretization is reduced to a few thousand degrees of freedom in the port-reduced SCRBE approximation. The online computation time is accordingly reduced from about an hour to only seconds, and thus the approach enables large-scale computation in many-query contexts such as interactive design or optimization. Further, our computational results for the microtruss structure indicate applications in stochastic homogenization and material failure identification, which may require many simulations for (say) random parameters and topology [30]. Another application is vibration analysis of structures as considered in [31].

The presented approach is an alternative to standard FE analysis of large componentbased structures such as bridges, microtrusses, or vehicle or building frames. However, we may consider any linear elliptic or parabolic [13] parameter-dependent partial differential equation, and thus problems in (say) heat transfer [32], acoustics [33], and electromagnetics may be considered as well.

\section{Endnotes}

${ }^{\text {a }}$ For non-symmetric, non-coercive, complex-valued, or parabolic problems additional elements are required for our a posteriori error estimation framework.

b We first apply the inverse map to physical coordinates to obtain reference coordinates, and then evaluate the function on the reference domain.

c To illustrate this latter application of the mapping, consider for example a vector field $(0,1)$ defined on $\hat{\Omega}_{1}$ in the frame $\left(\hat{x}_{1}, \hat{y}_{1}\right)$ in Figure 1 . We then consider this same vector field over $\Omega_{1}$ in Figure 2: by application of $\mathcal{T}_{1}^{\text {rot }}$ to the field $(0,1)$ we obtain an interpretation in the system frame $(x, y)$ which is consistent with the interpretation on the archetype domain - the field is parallel to the original $\hat{x}_{1}$ axis $\left(\right.$ for $\left.\Omega_{1}\right)$, and not parallel to the system $x$ axis.

${ }^{d}$ We start with the strong formulation on each component; we multiply by a test function and integrate by parts; we then add the equations on adjacent components and invoke flux continuity to cancel the corresponding port integral terms. In practice this is automatically accommodated by the variational formulation (23).

${ }^{\mathrm{e}}$ In this paper, we consider for $\mathcal{R}_{m, j}$ only rigid-body transformations; more general mappings and parametrized port deformations are also possible but is subject of future work. 
${ }^{\mathrm{f}}$ Note in the scalar-field case this simplifies to only the constant mode.

g Our current implementation does not recognize "free" parameters (Young's modulus, conductivity) and thus each set of component clones will contain components with different spatial orientation but identical ("non-docking") parameters.

${ }^{\mathrm{h}}$ With the current $\left(L^{2}(\beta)\right)^{d-1}$ orthogonalization of our port space bases, the $\ell_{2}$ norm of the residual coefficients is rather strong. We conjecture that the constant $C$ in (126) and (127) can be improved by consideration of an orthogonalization which provides a global Riesz basis (in the limit as the FE discretization parameter $h \rightarrow 0$ ) with respect to the $\left(H^{-1}(\Omega)\right)^{d}$ semi-norm.

${ }^{\mathrm{i}}$ The Von Mises stresses are calculated as $\sigma_{\mathrm{VM}}=\left(\frac{1}{2}\left(\left(\sigma_{11}-\sigma_{22}\right)^{2}+\left(\sigma_{22}-\sigma_{33}\right)^{2}+\right.\right.$ $\left.\left.\left(\sigma_{33}-\sigma_{11}\right)^{2}+6\left(\sigma_{12}^{2}+\sigma_{23}^{2}+\sigma_{31}^{2}\right)\right)\right)^{1 / 2}$.

j The formula for the maximum deflection $d(l)$ of a cantilever beam of length $l$, Young's modulus $E$, second moment of inertia $I$, and subject to tangential force $P$ at one end is $d(l)=\mathrm{Pl}^{3} /(3 E I)$.

${ }^{k}$ To reduce the error estimator in this case we would have to reduce the values in $\sigma_{\mathbb{A}}(\mu)$ by adding additional RB snapshots to the RB bubble spaces in the offline stage.

${ }^{1}$ For component 1 all instantiations have identical parameters, but there are two different component-local matrix blocks because we consider Dirichlet boundary conditions on all bottom ports of components located at the bottom of the microtruss structure.

m The $E_{i}$ correspond to component-wide Young's modulus, and hence these parameters are "free" in the sense discussed in the "Model reduction" section. Thus with a more complete treatment of effectively identical components we would have recovered $I_{\text {eff }}=4$ for this case.

\section{Additional file}

Additional file 1: A short video which illustrates the methodology of this paper is published together with this paper as prscrbe_movie.mp4.

Competing interests

The authors declare that they have no competing interests

Authors' contributions

JLE developed computational procedures and the associated $\mathrm{C}++$ implementation, contributed to the theoretical results, and drafted the manuscript. ATP developed computational procedures and theoretical results. All authors participated in the writing, review, and revision of the manuscript.

\section{Acknowledgements}

We are grateful to Dr. D. J. Knezevic for development of SCRBE library code, to Dr. D. B. P. Huynh for graphical system assembly and visualization software, and to Dr. S. Vallaghé for fruitful discussion. This work has been sponsored by the Research Council of Norway and ONR Grant N00014-11-0713.

Received: 9 August 2013 Accepted: 20 December 2013

Published: 29 January 2014

\section{References}

1. Abdelal GF, Abuelfoutouh N, Gad AH (2013) Finite element analysis for satellite structures. Springer, London

2. Egeland O, Haraldsen PO (1974) SESAM-69 - a general purpose finite element method program. Comput Struct 4: 41-68

3. Craig R, Bampton M (1968) Coupling of substructures for dynamic analyses. AIAA J 6(7): 1313-1319

4. Hurty WC (1964) On the dynamic analysis of structural systems using component modes In: First AIAA Annual Meeting. AIAA (American Institute of Aeronautics and Astronautics), Washington. AIAA paper, no. 64-487

5. Huynh DBP, Knezevic DJ, Patera AT (2013) A static condensation reduced basis element method: approximation and a posteriori error estimation. ESAIM: Math Model Numerical Anal 47(1): 213-251

6. Rozza G, Huynh DBP, Patera AT (2008) Reduced basis approximation and a posteriori error estimation for affinely parametrized elliptic coercive partial differential equations: application to transport and continuum mechanics. Arch Comput Methods Eng 15(3): 229-275

7. Binev P, Cohen A, Dahmen W, DeVore R, Petrova G, Wojtaszczyk P (2011) Convergence rates for greedy algorithms in reduced basis methods. SIAM J Math Anal 43(3): 1457-1472 
8. Haasdonk B (2013) Convergence rates of the pod-greedy method. ESAIM: Math Model Numerical Anal 47: 859-873

9. Bourquin F (1992) Component mode synthesis and eigenvalues of second order operators: discretization and algorithm. Math Model Numerical Anal 26(3): 385-423

10. Hetmaniuk UL, Lehoucq RB (2010) A special finite element method based on component mode synthesis. ESAIM: Math Model Numerical Anal 44(3): 401-420

11. Jakobsson H, Bengzon F, Larson MG (2011) Adaptive component mode synthesis in linear elasticity. Internat J Numer Methods Engrg 86(7): 829-844

12. Eftang JL, Patera AT (2013) Port reduction in parametrized component static condensation: approximation and a posteriori error estimation. Int J Numerical Methods Eng 96(5): 269-302

13. Vallaghé $S$ (2013) The static condensation reduced basis element method for parabolic problems. M3AS: Math Models Methods Appl Sci. http://augustine.mit.edu/methodology/papers/SV_M3AS_2013.pdf

14. Veroy K, Rovas DV, Patera AT (2002) A posteriori error estimation for reduced-basis approximation of parametrized elliptic coercive partial differential equations: "convex inverse" bound conditioners. ESAIM: Control, Optimisation Calculus Variations 8: 1007-1028

15. Quarteroni A, Valli A (1994) Numerical approximation of partial differential equations Springer Series in Computational Mathematics, vol. 23. Springer, Berlin

16. Bernardi C, Maday Y (1997) Spectral methods In: Handbook of Numerical Analysis, North-Holland, Amsterdam, pp 209-485

17. Kunisch K, Volkwein S (2002) Galerkin proper orthogonal decomposition methods for a general equation in fluid dynamics. SIAM J Numer Anal 40(2): 492-515

18. Isaacson E, Keller HB (1994) Computation of eigenvalues and eigenvectors, analysis of numerical methods

19. Hernández V, Román JE, Tomás A, Vidal V (2007) Krylov-Schur Methods in SLEPC. Technical report, Universidad Politecnica De Valencia. http://www.grycap.upv.es/slepc

20. Queheillalt DT, Wadley HNG (2005) Cellular metal lattices with hollow trusses. Acta Materialia 53: 303-313

21. Wadley HNG (2006) Multifunctional periodic cellular metals. Philos Trans R Soc A 364: 31-68

22. Wadley HNG, Fleck NA, Evans AG (2003) Fabrication and structural performance of periodic cellular metal sandwich structures. Composites Sci Technol 63: 2331-2343

23. Flügge W (1972) Tensor analysis and continuum mechanics. Springer, Berlin

24. Kirk BS, Peterson JW, Stogner RH, Carey GF (2006) libMesh: A C++ library for parallel adaptive mesh refinement/coarsening simulations. Eng Comput 22(3-4): 237-254

25. Knezevic DJ, Peterson JW (2011) A high-performance parallel implementation of the certified reduced basis method. Comput Methods Appl Mech Eng 200(13-16): 1455-1466

26. Timoshenko SP (1953) History of strength of materials. McGraw-Hill, New York

27. Saad Y (2003) Iterative methods for sparse linear systems, 2nd edn. Society for Industrial and Applied Mathematics, Philadelphia

28. Henson VE, Yang UM (2002) BoomerAMG: a parallel algebraic multigrid solver and preconditioner. Appl Numer Math 41(1): 155-177. Developments and trends in iterative methods for large systems of equations - in memoriam Rüdiger Weiss (Lausanne, 2000)

29. hypre: Scalable linear solvers. http://computation.IInl.gov/casc/linear_solvers/sls_hypre.html

30. Anantharaman A, Le Bris C (2011) A numerical approach related to defect-type theories for some weakly random problems in homogenization. Multiscale Model Simul 9(2): 513-544

31. Vallaghé S, Huynh DBP, Knezevic DJ, Patera AT (2013) Component-based reduced basis for eigenproblems. Comput Struct. http://augustine.mit.edu/methodology/papers/NHKP_CS_July2013.pdf

32. Vallaghé S, Patera AT (2012) The static condensation reduced basis element method for a mixed-mean conjugate heat exchanger model. SIAM J Sci Comput. http://augustine.mit.edu/methodology/papers/ VP_SISC_revised_May2013.pdf

33. Huynh DBP, Knezevic DJ, Patera AT (2013) A static condensation reduced basis element method: complex problems. Comput Methods Appl Mech Eng 259(0): 197-216

doi:10.1186/2213-7467-1-3

Cite this article as: Eftang and Patera: A port-reduced static condensation reduced basis element method for large component-synthesized structures: approximation and A Posteriori error estimation. Advanced Modeling and Simulation in Engineering Sciences 2013 1:3. 